BNL-94287-2010

Formal Report

\title{
The Physics of W and Z Bosons
}

June $24-25,2010$

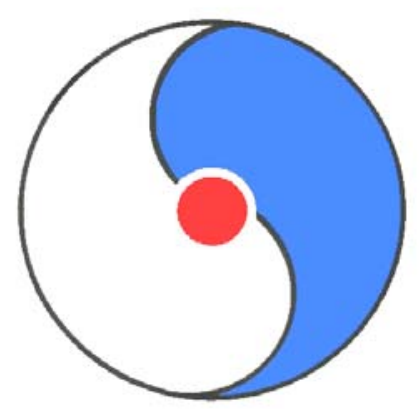

Organizers:

S. Dawson, K. Okada, A. Patwa, J. Qiu and B. Surrow

\section{RIKEN BNL Research Center}

Building 510A, Brookhaven National Laboratory, Upton, NY 11973-5000, USA 


\section{DISCLAIMER}

This work was prepared as an account of work sponsored by an agency of the United States Government. Neither the United States Government nor any agency thereof, nor any of their employees, nor any of their contractors, subcontractors or their employees, makes any warranty, express or implied, or assumes any legal liability or responsibility for the accuracy, completeness, or any third party's use or the results of such use of any information, apparatus, product, or process disclosed, or represents that its use would not infringe privately owned rights. Reference herein to any specific commercial product, process, or service by trade name, trademark, manufacturer, or otherwise, does not necessarily constitute or imply its endorsement, recommendation, or favoring by the United States Government or any agency thereof or its contractors or subcontractors. The views and opinions of authors expressed herein do not necessarily state or reflect those of the United States Government or any agency thereof.

Notice: This manuscript has been authored by employees of Brookhaven Science Associates, LLC under Contract No. DE-AC02-98CH10886 with the U.S. Department of Energy. The publisher by accepting the manuscript for publication acknowledges that the United States Government retains a non-exclusive, paid-up, irrevocable, world-wide license to publish or reproduce the published form of this manuscript, or allow others to do so, for United States Government purposes. 


\section{Preface to the Series}

The RIKEN BNL Research Center (RBRC) was established in April 1997 at Brookhaven National Laboratory. It is funded by the "Rikagaku Kenkyusho" (RIKEN, The Institute of Physical and Chemical Research) of Japan. The Memorandum of Understanding between RIKEN and BNL, initiated in 1997, has been renewed in 2002 and again in 2007. The Center is dedicated to the study of strong interactions, including spin physics, lattice QCD, and RHIC physics through the nurturing of a new generation of young physicists.

The RBRC has both a theory and experimental component. The RBRC Theory Group and the RBRC Experimental Group consists of a total of 25-30 researchers. Positions include the following: full time RBRC Fellow, half-time RHIC Physics Fellow, and full-time, post-doctoral Research Associate. The RHIC Physics Fellows hold joint appointments with RBRC and other institutions and have tenure track positions at their respective universities or BNL. To date, RBRC has $\sim 50$ graduates of which 14 theorists and 6 experimenters have attained tenure positions at major institutions worldwide.

Beginning in 2001 a new RIKEN Spin Program (RSP) category was implemented at RBRC. These appointments are joint positions of RBRC and RIKEN and include the following positions in theory and experiment: RSP Researchers, RSP Research Associates, and Young Researchers, who are mentored by senior RBRC Scientists. A number of RIKEN Jr. Research Associates and Visiting Scientists also contribute to the physics program at the Center.

RBRC has an active workshop program on strong interaction physics with each workshop focused on a specific physics problem. In most cases all the talks are made available on the RBRC website. In addition, highlights to each speaker's presentation are collected to form proceedings which can therefore be made available within a short time after the workshop. To date there are ninety seven proceeding volumes available.

A 10 teraflops RBRC QCDOC computer funded by RIKEN, Japan, was unveiled at a dedication ceremony at BNL on May 26, 2005. This supercomputer was designed and built by individuals from Columbia University, IBM, BNL, RBRC, and the University of Edinburgh, with the U.S. D.O.E. Office of Science providing infrastructure support at BNL. Physics results were reported at the RBRC QCDOC Symposium following the dedication. QCDSP, a 0.6 teraflops parallel processor, dedicated to lattice QCD, was begun at the Center on February 19, 1998, was completed on August 28, 1998, and was decommissioned in 2006. It was awarded the Gordon Bell Prize for price performance in 1998.

N. P. Samios, Director

March 2010

*Work performed under the auspices of U.S.D.O.E. Contract No. DE-AC02-98CH10886. 



\section{CONTENTS}

Preface to the Series

Introduction

First Measurement of $\mathrm{W}+/ \mathrm{W}$ - Boson Production at STAR in Polarized pp Collisions at RHIC Jan Balewski

First Observations at PHENIX of W Production From Polarized pp Collisions at RHIC David Kawall

ResBos and RhicBos: $\mathrm{Q}_{\mathrm{T}}$ Resummation for (un)polarized EW Boson Production Pavel Nadolski

Precision Determination of MW from Observables at Hadron Colliders Alessandro Vicini

W and $Z$ Physics from HERA

David South

Measurement of W Boson Mass at D0

Junjie $Z h u$

Measurement of the $\mathrm{W}$ Boson Mass at CDF

Ashutosh Kotwal

Monte Carlo Modelling Issues for W Measurements Jan Stark

Theoretical Issues in Monte Carlo Modelling for W/Z Production

Jan Winter

W Boson Physics and Global Analysis of PDFs

C. -P. Yuan

Prospects and Future of the STAR W Program

Collisions at RHIC

Joe Seele

Prospects of the Forward W Measurement in Polarized pp Collisions at the RHIC-PHENIX

Experiment

Yoshi Fukao

$\mathrm{A}_{\mathrm{N}}$ of W Production in Polarized pp Collisions

Zhong-Bo Kang

Precision Measurement of the W Mass and New Physics

Ulrich Baur

W Measurements and Prospects at ATLAS

Kevin Black

Current Status and Prospects for W and Z Cross Section Measurements at CMS

Carsten Magass

The Status of NNLO Tools for W/Z Production at Hadron Colliders

Frank Petriello

List of Registered Participants

Agenda

Additional RIKEN BNL Research Center Proceeding Volumes

Contact Information 



\section{Introduction}

A two-day workshop on "The Physics of W and Z Bosons" was held at the RIKEN BNL Research Center at Brookhaven National Laboratory on June 24-25, 2010.

With the recent release of the first measurement of $W$ bosons in proton-proton collisions at RHIC and the first observation of W events at the LHC, the workshop was a timely opportunity to bring together experts from both the high energy particle and nuclear physics communities to share their ideas and expertise on the physics of $\mathrm{W}$ and $\mathrm{Z}$ bosons, with the aim of fully exploring the potential of the $\mathrm{W} / \mathrm{Z}$ physics programs at RHIC and the LHC.

The focus was on the production and measurement of $W / Z$ bosons in both polarized and unpolarized proton-proton collisions, and the role of $\mathrm{W} / \mathrm{Z}$ production in probing the parton flavor and helicity structure of the colliding proton and in the search for new physics. There were lively discussions about the potential and future prospects of $\mathrm{W} / \mathrm{Z}$ programs at RHIC, Tevatron, and the LHC.

Organizers: S. Dawson, K. Okada, P. Patwa, J. Qiu and B. Surrow 


\section{First Measurement of $\mathbf{W}+/ \mathbf{W}-$ Boson Production at STAR in Polarized pp Collisions at RHIC \\ Jan Balewski (For the STAR Collaboration) \\ Massachusetts Institute of Technology \\ balewski@mit.edu}

The STAR experiment has acquired its first set of $W$-boson events from collisions of longitudinally polarized protons at $\sqrt{s}=500 \mathrm{GeV}$. The STAR Electromagnetic Calorimeter triggered on electrons/positrons from the weak decay of the $W$ and provided the energy of the lepton, while the STAR Time Projection Chamber allowed reconstruction of the lepton track and its charge sign. The QCD physics background was suppressed by isolation cuts around a candidate lepton track as well as vetoing on transverse energy opposite in azimuth. In the standard model leadingorder $W^{ \pm}$production is through $u+\bar{d} \rightarrow W^{+}$and $d+\bar{u} \rightarrow W^{-}$. These interactions are ideal tools to study the spin-flavor structure of the proton, because the spin-dependent $W$ production cross section $\Delta \sigma=\sigma(\vec{p} p)-\sigma(\overleftarrow{p} p)$ depends strongly on the polarization of the quark and anti-quark in the proton, with $\vec{p}(\overleftarrow{p})$ representing a proton with its spin aligned with (against) its momentum. We will present the status of the STAR measurement of $A_{L}=\Delta \sigma /(\sigma(\vec{p} p)+\sigma(\grave{p} p))$ for mid-rapidity charge separated $W^{+}$and $W^{-}$. 


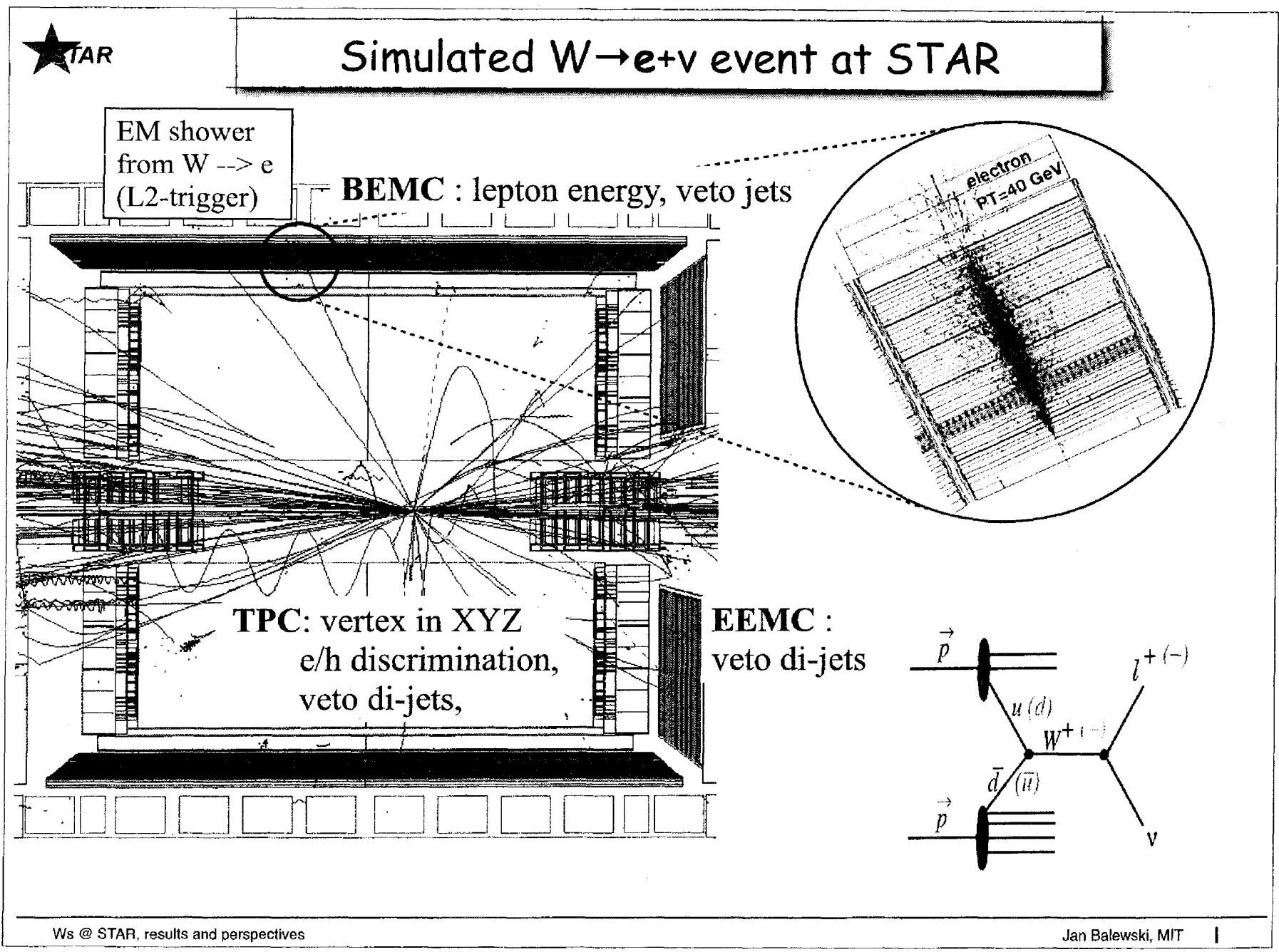




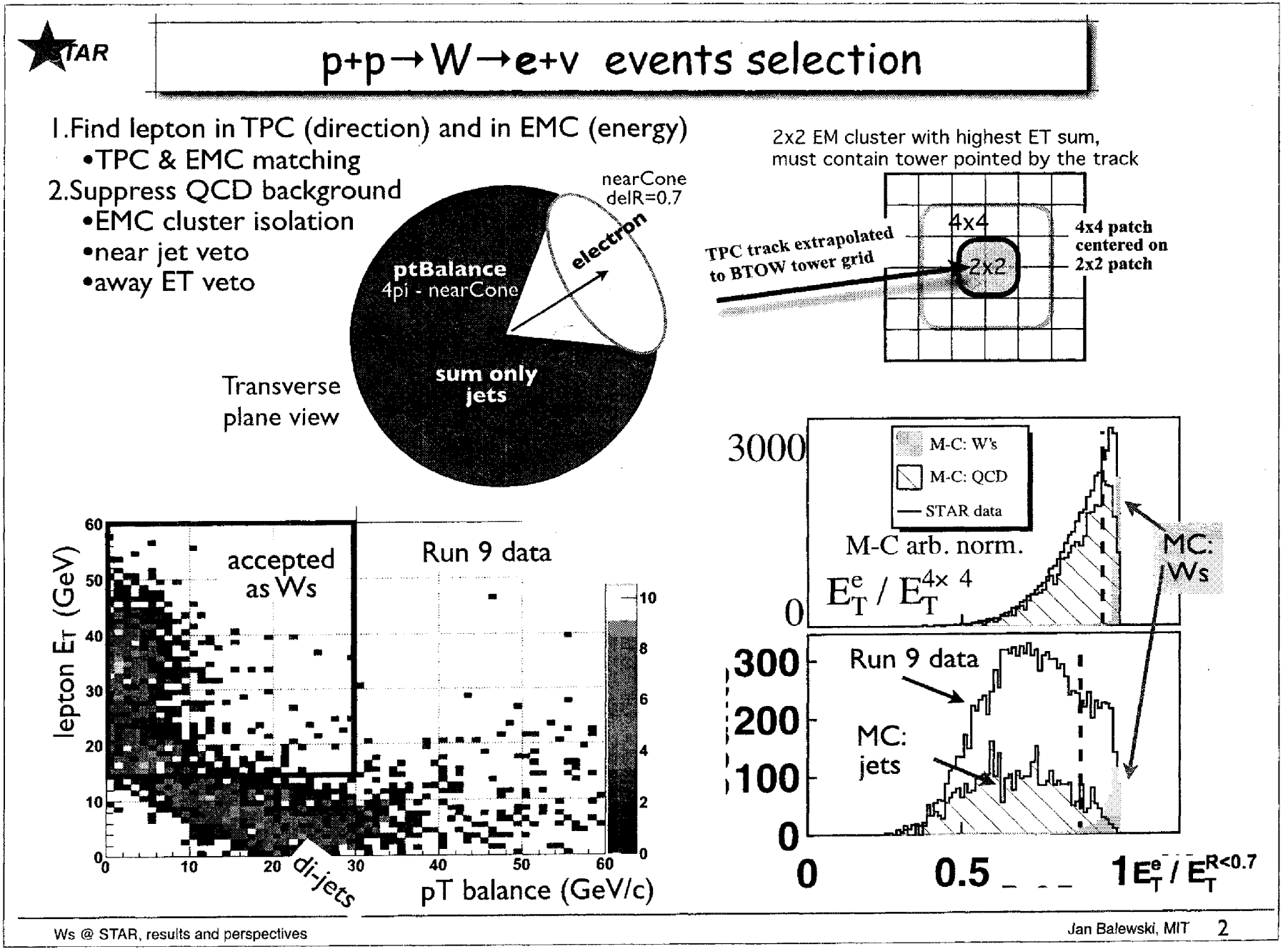




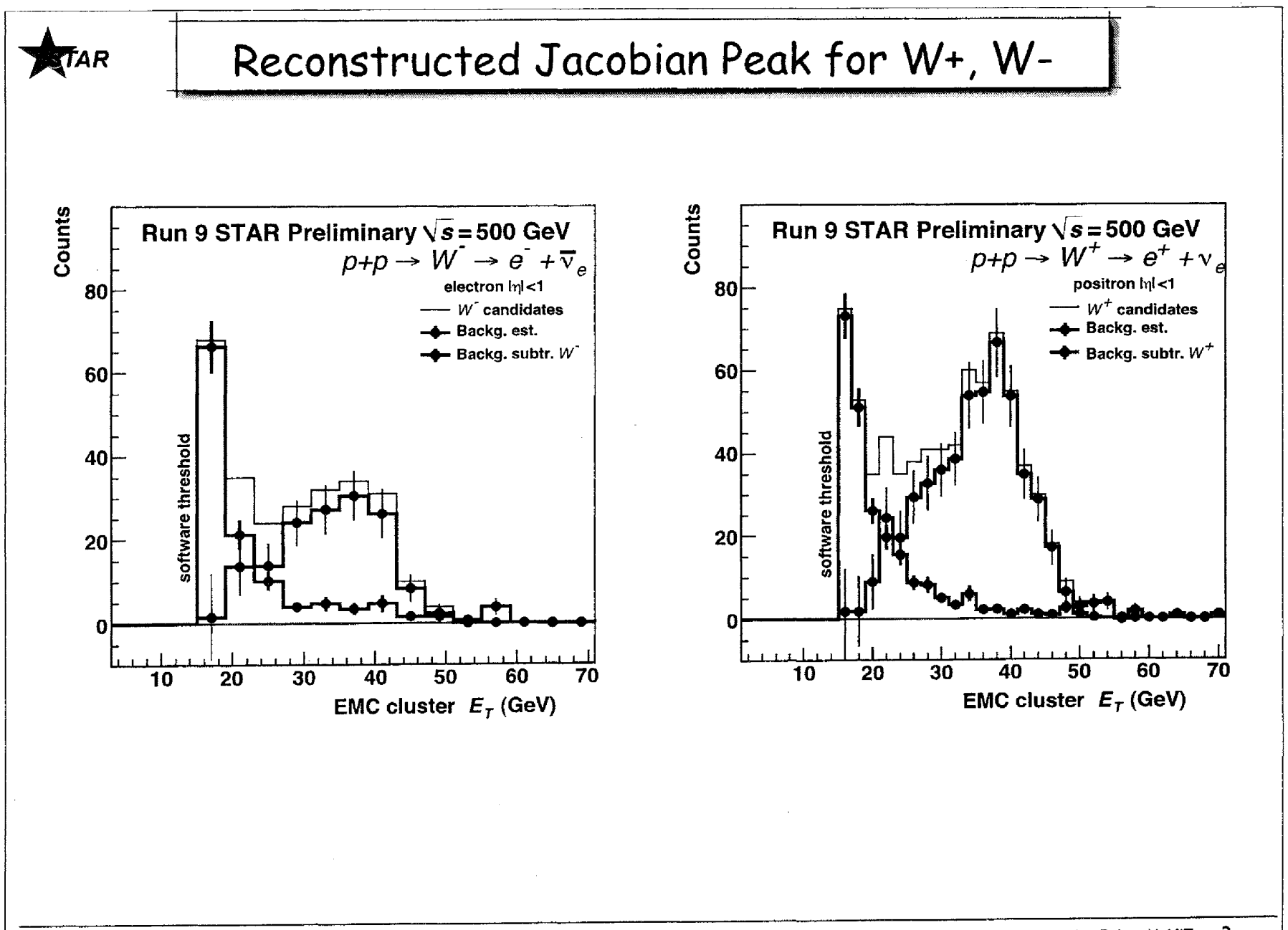




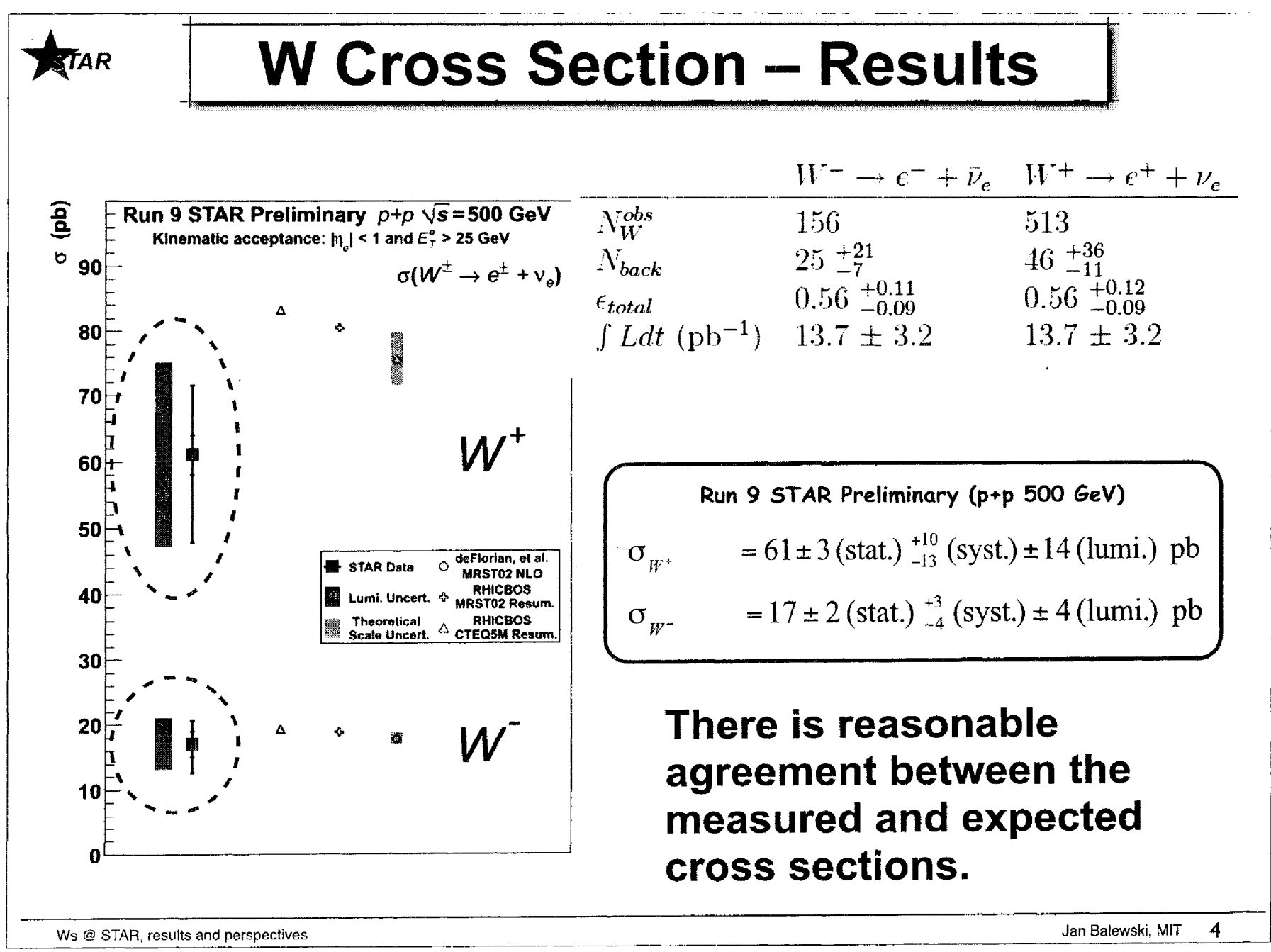




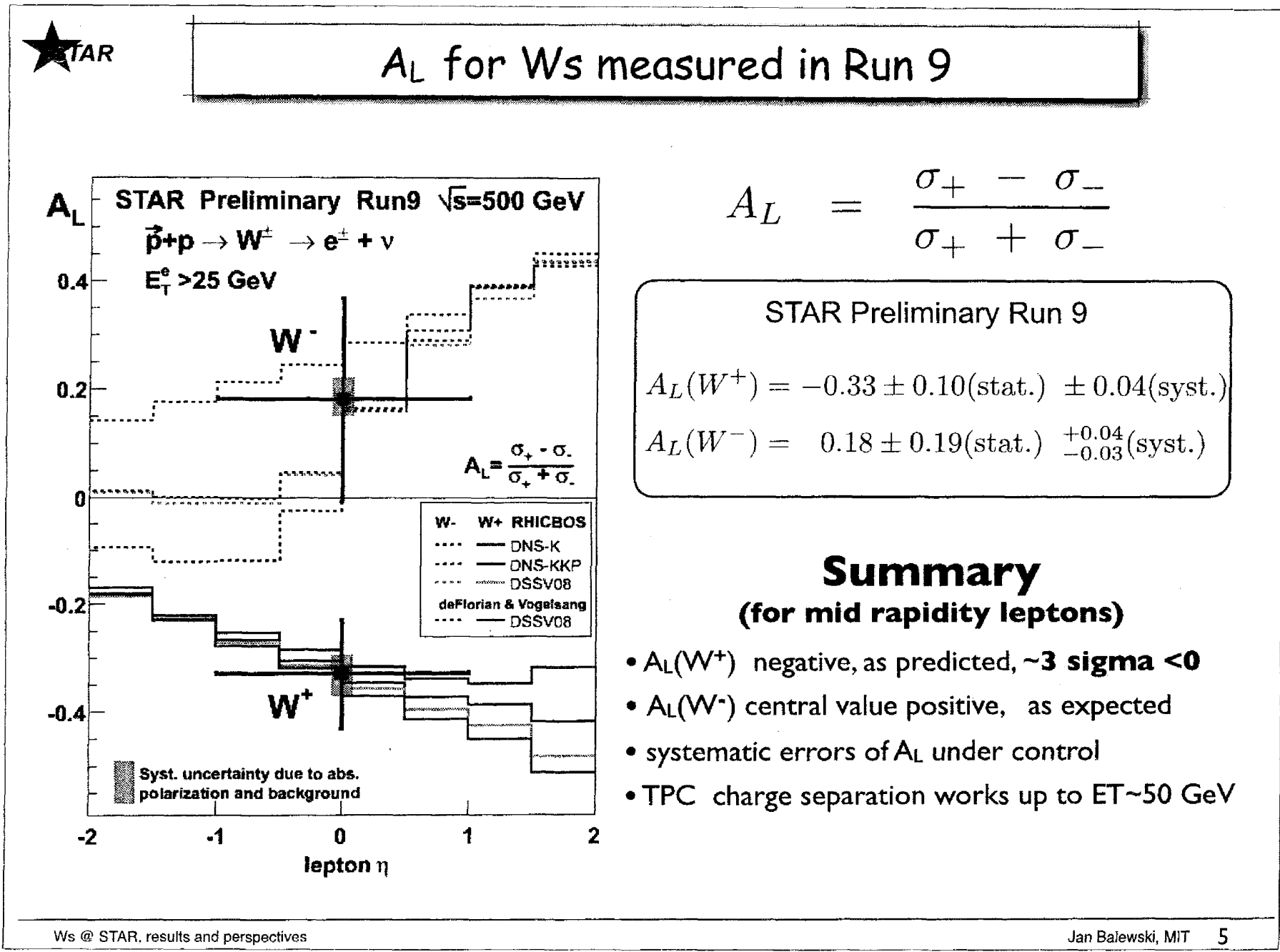


First Observations at PHENIX of $W$ Production from Polarized $p p$ Collisions at RHIC

Dave Kawall, RIKEN-BNL Research Center and University of Massachusetts Amherst on behalf of the PHENIX Collaboration

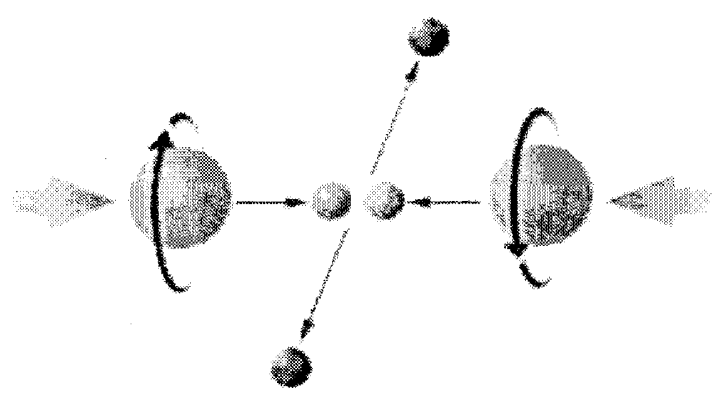

The evidence for $p p \rightarrow W+X \rightarrow e+X^{\prime}$ in polarized $p p$ collisions at $\sqrt{s}=500 \mathrm{GeV}$ in the PHENIX detector at RHIC is presented. The physics importance, analysis strategy, and preliminary results on the single longitudinal, parity-violating spin asymmetry $A_{L}^{W^{+}}$are also presented. 


\section{Motivation for Spin Physics with $W$ s at RHIC}

- Key measurement of spin program : flavor separated, polarized PDFs $\Delta \bar{u}(x)$ and $\Delta \bar{d}(x)$

- Semi-inclusive polarized DIS experiments (SMC, HERMES, COMPASS) have made such measurements

- STAR and PHENIX can do it exploiting maximal-parity violation in $W$ production in polarized $p p$ collisions Measurements made at high scale $\left(M_{W}^{2}>6000 \mathrm{GeV}^{2}\right)$

No uncertainty from fragmentation (couplings of $W$ well known), no higher twist effects

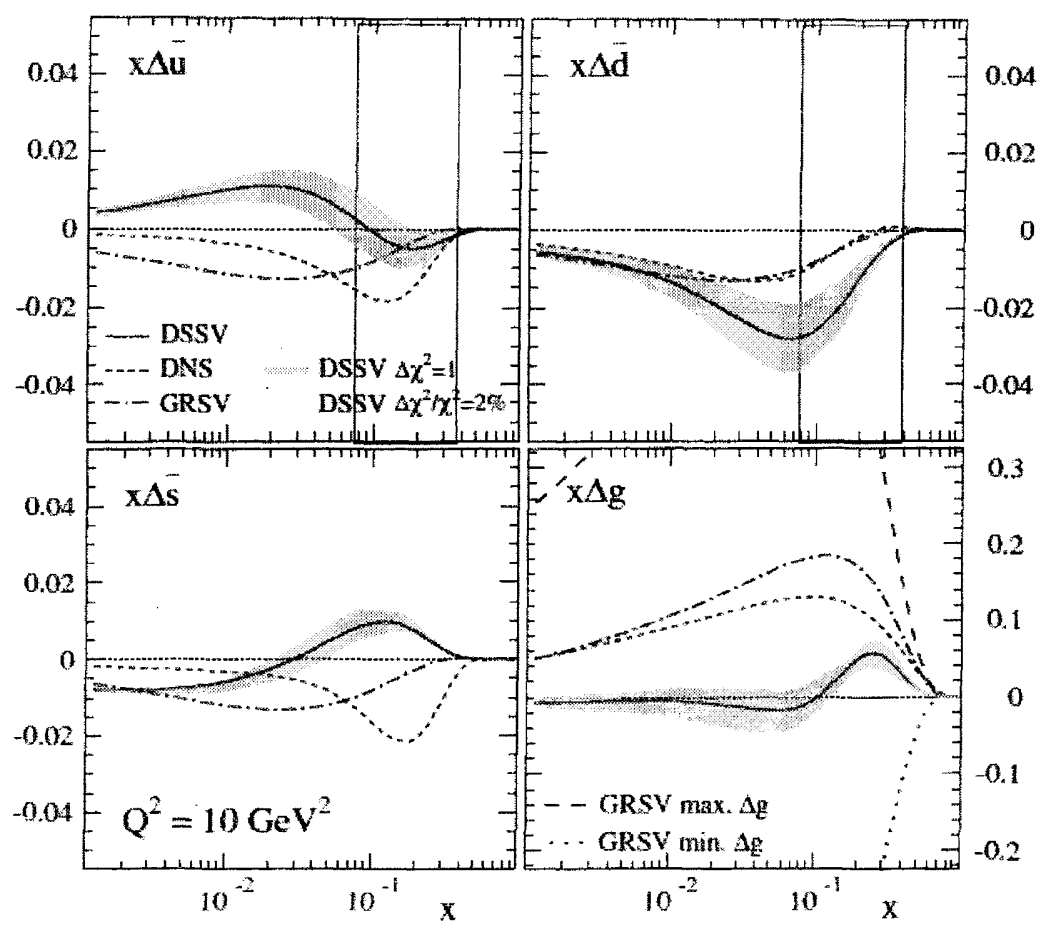

- Unpol. PDFs known to about $10 \%$

- Theoretical uncertainties small (NLO+resummation)

- Robust extraction of $\Delta \bar{u}(x)$ and $\Delta \bar{d}(x)$

* Can also measure ratio $\bar{u}(x) / \bar{d}(x)$

$\leftarrow$ D. de Florian, R. Sassot, M. Stratmann, and W. Vogelsang, Phys. Rev. Lett. 101, 072001 (2008) (At $Q^{2}=10 \mathrm{GeV}^{2}$ ) 


\section{PHENIX Central Arm Spectrometers}

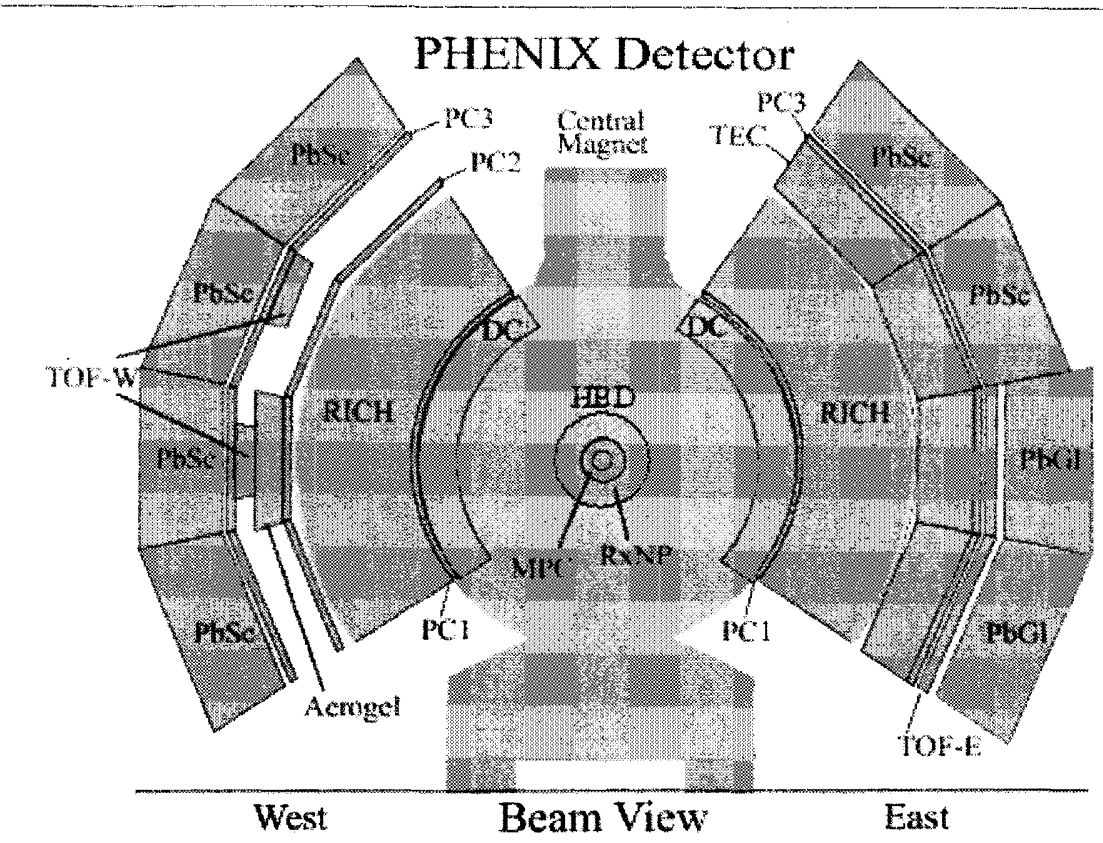

- Electromagnetic calorimeter (EMCal finely segmented :

$\Delta \phi \times \Delta \eta \approx 0.01 \times 0.01$

- Calibrated with $M_{\gamma \gamma}$ of $\pi^{0}$ at high $p_{T}$

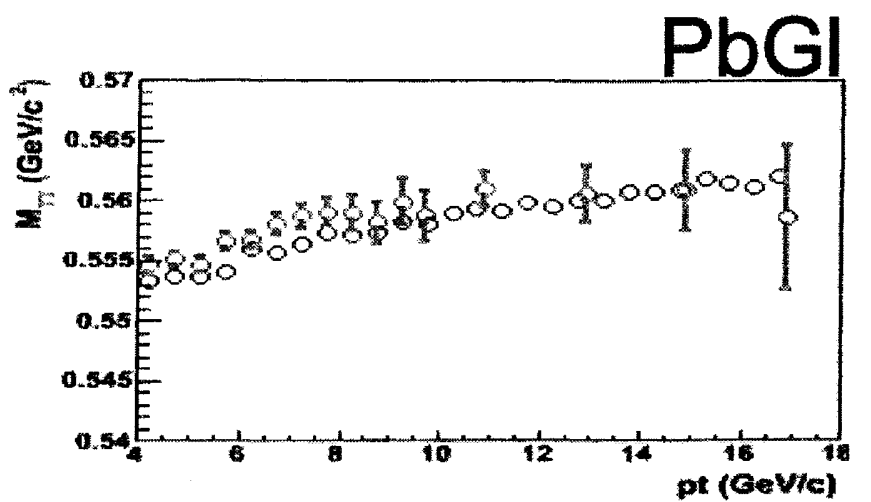

$\Rightarrow$ Focus on $\overrightarrow{p p} \Rightarrow W^{ \pm}+X \Rightarrow e^{ \pm}+X^{\prime}$

- Detect high $E e^{ \pm}$in central arms of PHENIX

- Acceptance of each arm : rapidity $|\eta|<0.35$ $(70<\theta<110), \Delta \phi=\pi / 2$

- Tracking: Charged tracks measured $\mathrm{i}$ Drift Chamber (DC) and Pad Cham ber(PC1)

- Vertex cut : $|z|<30 \mathrm{~cm}$

- $\int \vec{B} \cdot d \vec{l}=0.78$ Tesla-meters 

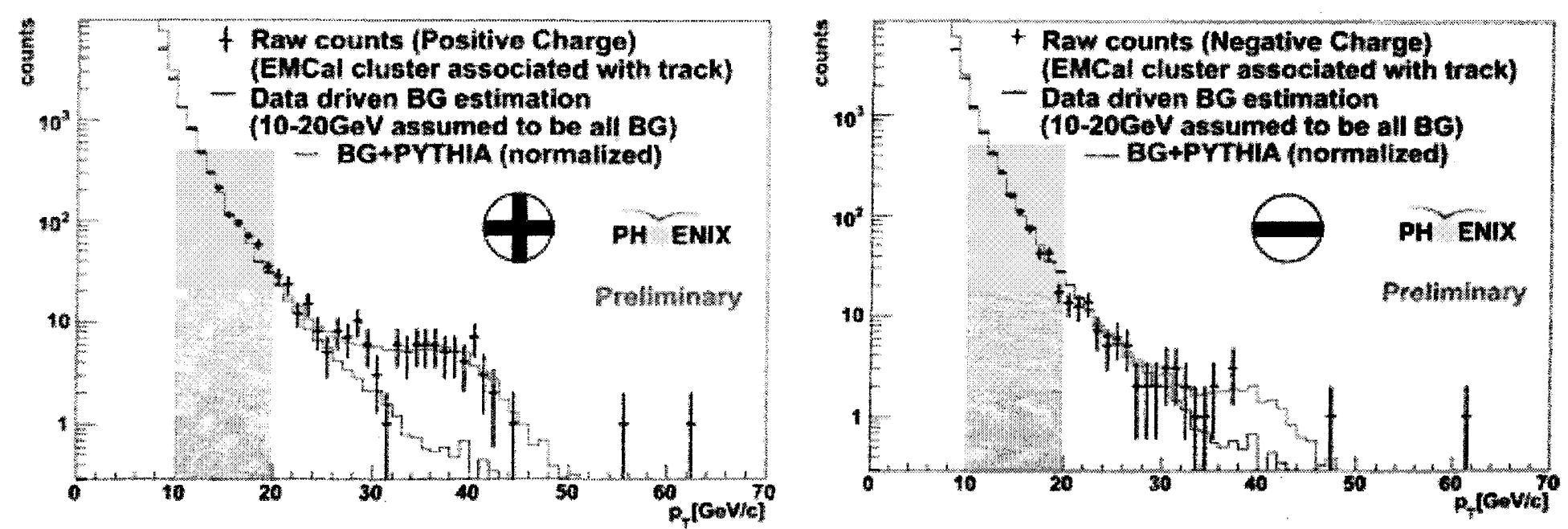

To determine background under signal region (30-50 GeV) :

- Take measured $\pi^{0}+\gamma$ spectrum $\times$ conversion prob + accidental matching track $\otimes$ acceptance

- Add charged hadrons (NLO) $\otimes$ detector response (GEANT) $+e^{ \pm}$from FONLL $c / b$ decays

- Normalize $h^{ \pm}$component so total background matches data in range 10-20 GeV

- Black histogram : background estimate; largest component from $\pi^{0}+\gamma, h^{ \pm}$slightly less 


$$
\text { Parity-Violating Single Spin Asymmetry } A_{L}\left(\vec{p} p \rightarrow W^{+} \rightarrow e^{+}\right)
$$

- Preliminary result, using $P_{B}=0.38 \pm 0.04$ and $P_{Y}=0.40 \pm 0.04 \quad(\delta P / P=9.2 \%)$

- Raw asymmetry in background region $(12-20 \mathrm{GeV} / \mathrm{c})$ consistent with $0: \epsilon_{\mathrm{raw}}^{\mathrm{Bkgd}}=0.035 \pm 0.047$

- Raw asymmetry in signal region (30-50 GeV/c) inconsistent with $0: \epsilon_{\text {raw }}^{\text {Signal }}=-0.29 \pm 0.11$

- $A_{L}=\frac{1}{P} \times \epsilon_{\text {raw }} \times D$, correct for dilution of $A_{L}$ by $Z$ and $Q C D$ background $(D=1.11 \pm 0.04)$

$$
A_{L}\left(\overrightarrow{p p} \rightarrow W^{+} \rightarrow e^{+}\right)=-0.83 \pm 0.31
$$

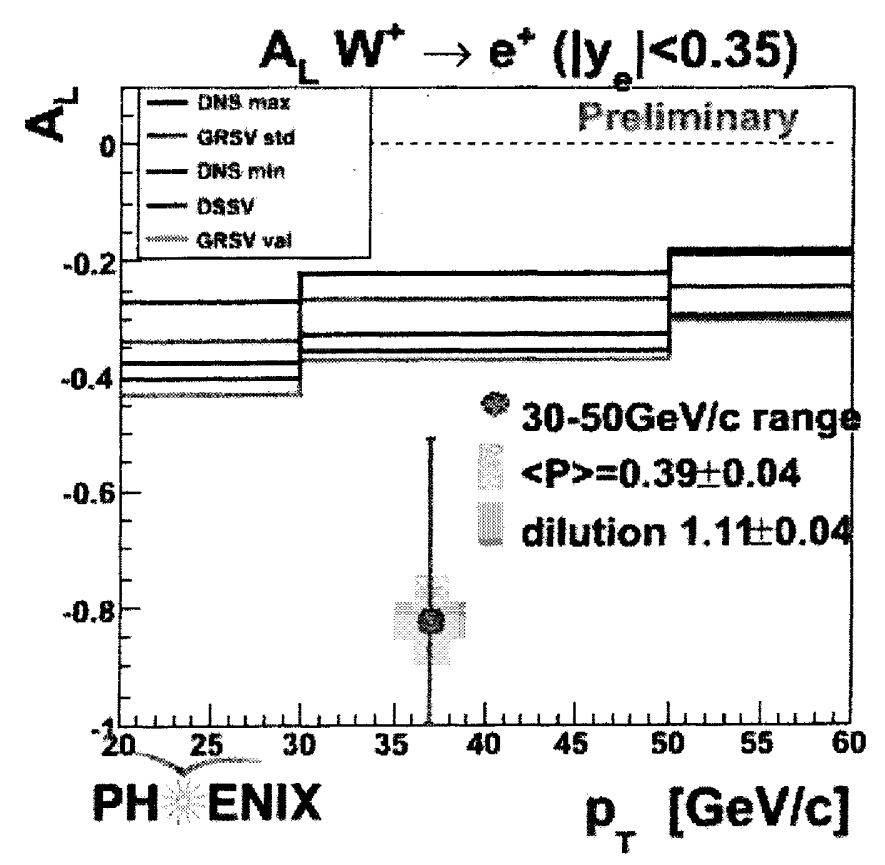


- Developed analysis techniques to isolate $W \rightarrow e$ signal above backgrounds

- Clear evidence for $W^{ \pm} \rightarrow e^{ \pm}$at $|\eta|<0.35$ in PHENIX central arms

- Preliminary determination of single-spin parity-violating asymmetry : $A_{L}^{W}\left(\vec{p} p \rightarrow W^{+} \rightarrow e^{+}\right)=-0.83 \pm 0.31$

- Analysis underway for cross-section estimates, final $A_{L}^{W^{ \pm}}$determinations

- Upgrades will help refine analysis, add acceptance and new physics channels :

- Si Barrel vertex detectors in PHENIX central arms

Muon arms : RPCs + muon trigger upgrade : $W \rightarrow \mu$ signal $1.2<\left|\eta_{\mu}\right|<2.2$

- C-AD getting closer to design luminosity at $\sqrt{500} \mathrm{GeV}, \approx 40 \%$ polarization

- Will need $300+\mathrm{pb}^{-1}$ integrated luminosity, $60 \%$ polarization to meet goals of program 


\section{ResBos and RhicBos}

$Q_{T}$ resummation for (un)polarized EW boson production

Pavel Nadolsky

Southern Methodist University

June 24,2010 


\title{
Objectives of the talk
}

\author{
An overview of physics in
}

- ResBos: Resummation for electroweak Bosons and their decays in unpolarized $p p$ or $p \bar{p}$ collisions

- RhicBos = ResBos adapted to compute longitudinal single-spin and double-spin asymmetries in leptonic decays of $\gamma^{*}, W, Z$ in $p p$ collisions

- Single-spin asymmetries in hadronic decays of $W$ bosons - a useful measurement complementary to the leptonic mode

Interruptions and questions are welcome! 


\section{Today's focus is on...}

- unpolarized parton distributions:

$$
f_{a / p}(x, Q) \equiv f_{a / p}^{+/+}(x, Q)+f_{a / p}^{-/+}(x, Q)
$$

- longitudinally polarized parton distributions:

$$
\Delta f_{a / p}(x, Q) \equiv f_{a / p}^{+/+}(x, Q)-f_{a / p}^{-/+}(x, Q)
$$

- unpolarized cross sections:

$$
\sigma=\frac{1}{2}\left[\sigma\left(p^{\rightarrow} p\right)+\sigma\left(p^{\leftarrow} p\right)\right]
$$

- single-spin cross sections ( $\neq 0$ if $V-A$ interaction):

$$
\Delta_{L} \sigma=\frac{1}{2}\left[\sigma\left(p^{\rightarrow} p\right)-\sigma\left(p^{\leftarrow} p\right)\right]
$$

- single-spin asymmetry as a function of $W$ boson rapidity

$$
A_{L}(y) \equiv \frac{d \Delta_{L} \sigma / d y}{d \sigma / d y}
$$
$y$ : 


\section{Two classes of subprocesses with $W$ bosons}

1: Resonant (s-channel) $W$ boson production

- dominant parity-violating process at $Q \approx M_{W}$

- Leptonic decays: $\operatorname{Br}\left(W \rightarrow e \nu_{e}\right) \approx 10.8 \%$ RhicBos

- Hadronic decays: $\operatorname{Br}(W \rightarrow$ hadrons $) \approx 67 \%$

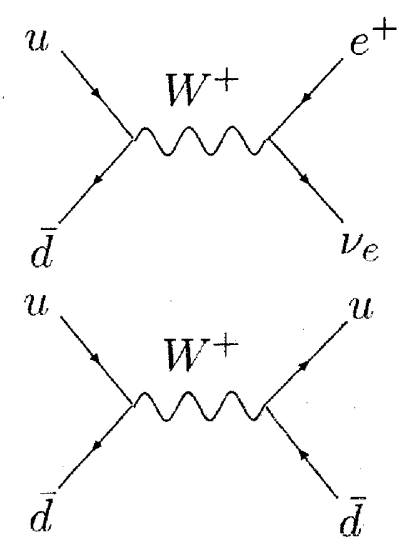

2: Non-resonant scattering into a dijet final state, mediated by $\gamma^{*}, W, Z$, and $g$, and interference terms
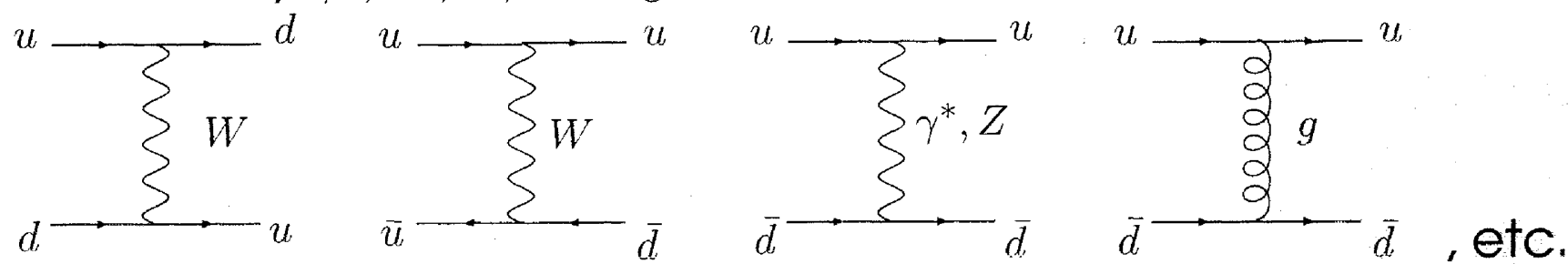


\section{Related publications}

\section{On-shell $W$ boson production}

1. C. Bourrely, J. Soffer, PLB 314, 132 (1993); Nucl.Phys. B423, 329 (1994)

2. A. Weber, Nucl. Phys. B 403, 545 (1993)

3. P. Nadolsky, hep-ph/9503419

4. T. Gehrmann, Nucl. Phys. B534, 21(1998)

5. M. Gluck, A. Hartl, and E. Reya, Eur. Phys. J. C19, 77 (2001)

\section{Leptonic decay mode}

1. B. Kamal, Phys. Rev. D57, 6663 (1998)

2. P. Nadolsky and C.-P. Yuan, Nucl. Phys. B666, 3 and 31 (2003)

\section{Dijet mode}

1. H. Haber and G. Kane, Nucl. Phys. B146,109 (1978)

2. F. Paige, T. L. Trueman, T. Tudron, Phys. Rev. D 19, 935 (1979)

3. C. Bourrely, J. P. Guillet, and J. Soffer, Nucl. Phys. B361, 72 (1991)

4. S. Arnold, A. Metz, V. Vogelsang, arXiv:0807.3688; S. Arnold, K. Goeke, A. Metz, P. Schweitzer, W. Vogelsang, Eur.Phys.J.ST 162 (2008) 


\section{Data-driven search for resonant $W \rightarrow$ jet + jet contributions}

$A_{L}(y)$ is most accessible in the signal region:

$$
Q=M_{W} \pm 10-15 \mathrm{GeV}, p_{T j} \gtrsim 25 \mathrm{GeV},\left|y_{1 j}-y_{2 j}\right| \lesssim 1
$$

The measurement can be based on the following strategy:

1. Discard events with gluon-like jets (wide, large multiplicity) to the best of one's ability

2. Precisely measure the smooth background outside of the signal region

3. Use this measurement to predict and subtract the background inside the signal region

4. Look for a large $A_{L}(y)$ at $y>+1$

(driven by a large $\Delta u(x) / u(x)$ at $x \rightarrow 1$ )

5. Measure moderate $A_{L}(y)$ at $y<-1$

to constrain $\Delta \bar{d}(x) / \bar{d}(x)$ at $x<0.1$ 


\title{
Precision determination of MW from observables at hadron colliders
}

\author{
Alessandro Vicini \\ Dipartimento di Fisica, Università degli Studi di Milano and INFN, Sezione di Milano, \\ Via Celoria 16, 20133 Milano (Italy)
}

\begin{abstract}
The measurement of MW will probably reach the $15 \mathrm{MeV}$ level at the Tevatron. The measurement of this pseudo-observable heavily involves theoretical ingredients. In order to attempt an estimate of the final theoretical systematic uncertainty, one needs a classification of the impact of different classes of radiative corrections in terms of shifts of the final value of $\mathrm{MW}$.

Once known higher order effects have been estimated and possibly included in the data analysis, it will be possible, by comparing the predictions of different codes, to study the remaining sources of theoretical uncertainty and to obtain a final theoretical systematic error on MW.

Fixed order calculations provide the first basic estimates of the relevant cross sections, but a realistic simulation shows which effects survive after e.g. the convolution with multiple gluon/photon emission and with the smearing of lepton momenta or the leptonphoton recombination.

In this talk I will describe a simple procedure to perform a template fit of theoretical distributions, treated as pseudo-data, deriving a classification of the impact of $M W$ of different classes of radiative corrections. This procedure will be applied to study: i) change of EW input scheme, use of factorized expressions, EW higher orders; ii) QCD corrections by different codes; iii) combination of $\mathrm{QCD}+\mathrm{EW}$ corrections; iv) PDF uncertainties.
\end{abstract}


The template fitting procedure

A distribution computed with a given set of radiative corrections and with a given value $\mathrm{MW}_{0}$ is treated as a set of pseudo-data. The templates are prepared in Born approximation, using 100 values of $\mathrm{MW}_{i}$ Each template is compared to the pseudo-data and a distance is measured.

$$
\chi_{i}^{2}=\sum_{j=1}^{N_{b i n s, s}} \frac{\left(O_{j}^{\text {data }}-O_{j}^{\text {templ }=i}\right)^{2}}{\left(\sigma_{j}^{\text {data }}\right)^{2}} \quad i=1, \ldots, N_{\text {templ }}
$$

The template that minimizes the distance is considered as the preferred one and the value of $\mathrm{MW}$, used to generate it, is the measured MW.

The difference $M W-M W_{0}$ represents the shift induced on the measurement of the $\mathrm{W}$ mass by including that specific set of radiative corrections

The distributions used in the evaluation of $\chi_{i}^{2}$ in general do not have the same normalization. It is also possible to compare distributions that have been normalized to their respective cross-sections, to appreciate the role of the shape differences.

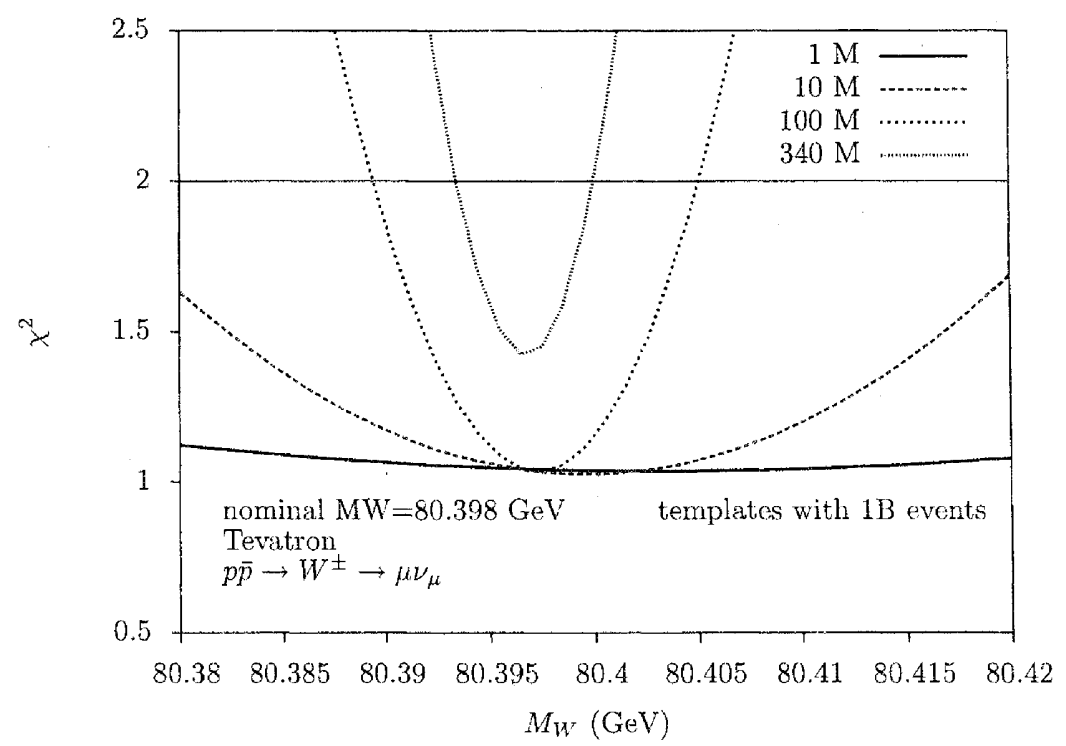

Figure 1: Pseudodata in Born approximation, fitted with Born templates. The nominal MW value used to generated the pseudodata is found from the fitting procedure, with increasing accuracy depending on the number of events in the pseudodata sample. The $\Delta \chi^{2}=1$ rule, valid in this exercise, determines the uncertainty in the fitting procedure associated to the extraction of a preferred value of MW. 
Effect of higher order corrections in the $\alpha(0)$ input scheme

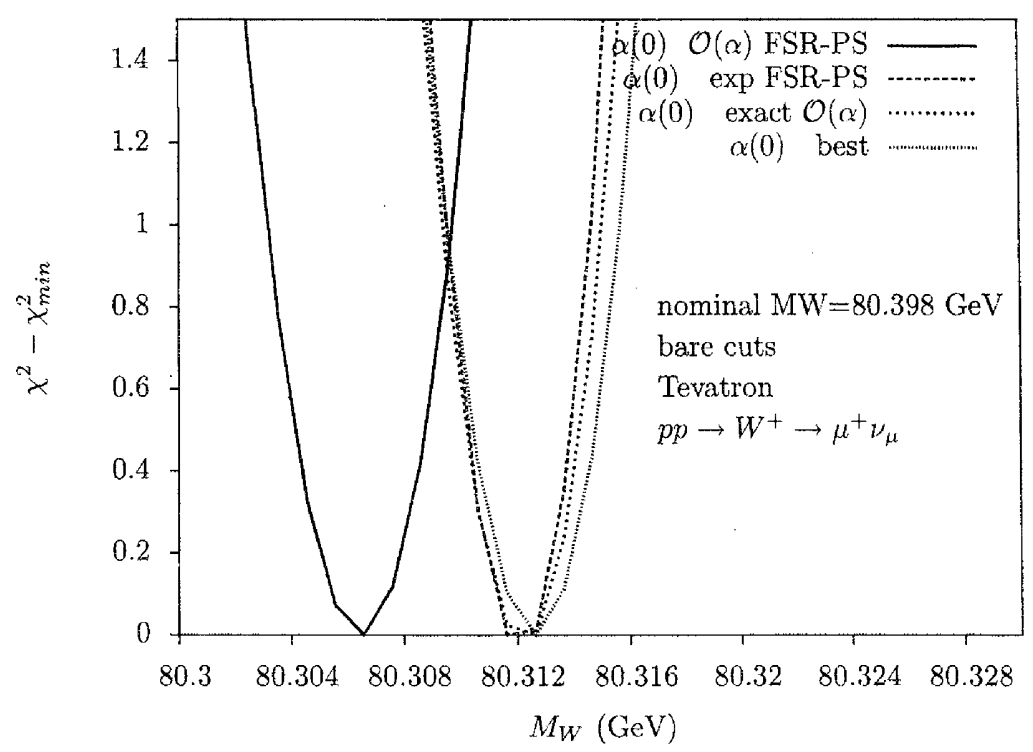

Figure 2: Impact of different higher order corrections, all evaluated in the $\alpha(0)$ input scheme, on the extraction of a preferred MW value.

The templates used to evaluate the effects in fig. 2 have been prepared using HORACE, Born approximation, $\alpha(0)$ input scheme, 10 billions of events, $80.248 \leq M W \leq 80.348$ GeV. 


\section{Effect of different EW input scheme choices}

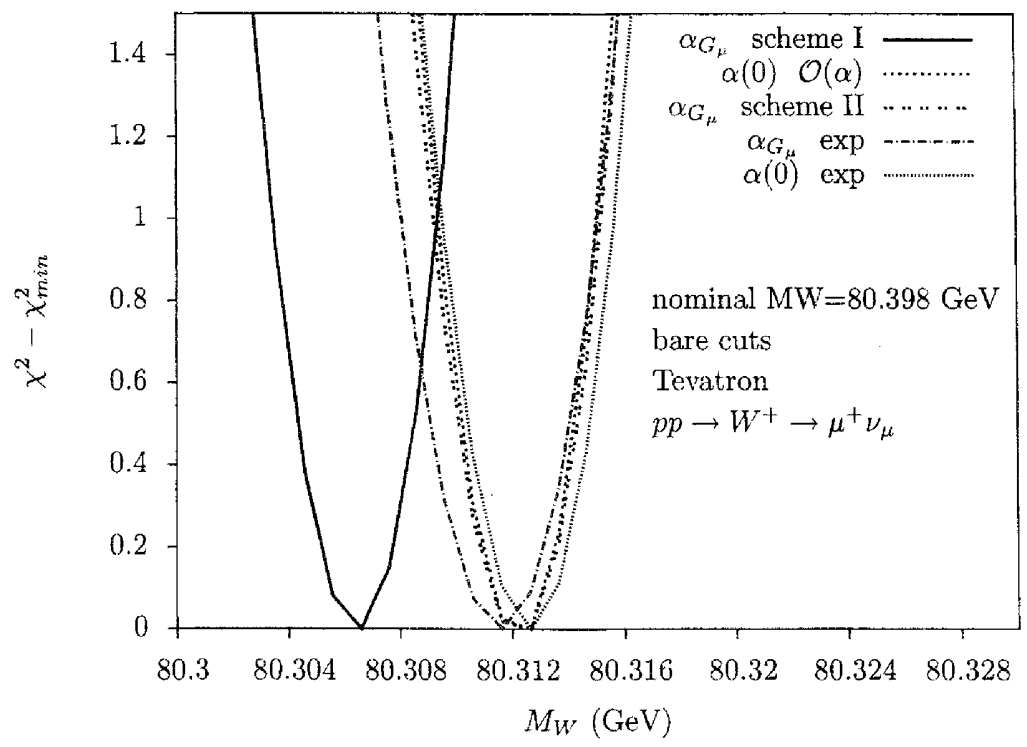

Figure 3: Impact of different EW input scheme choices on the extraction of a preferred MW value, in different perturbative approximations.

The templates used to evaluate the effects in fig. 3 have been prepared using HORACE, Born approximation, $\alpha(0)$ input scheme, 10 billions of events, $80.248 \leq M W \leq 80.348$ $\mathrm{GeV}$. 


\section{Effect of different methods to include multiple gluon radiation}

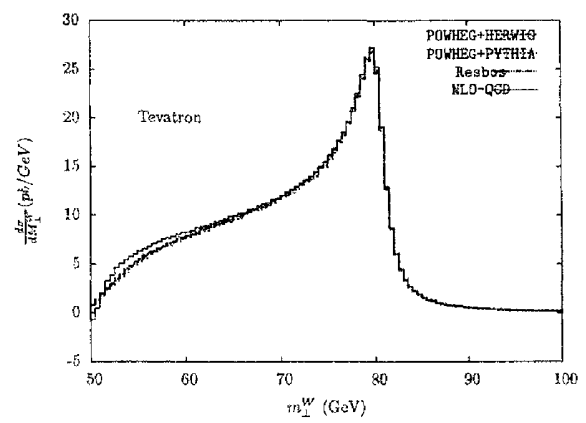

Figure 4: Transverse mass distribution obtained with different code that share NLO-QCD accuracy but include multiple gluon radiation with different approaches: analytical resummation (Resbos) or QCD Parton Shower with different ordering algorithms (HERWIG vs PYTHIA)

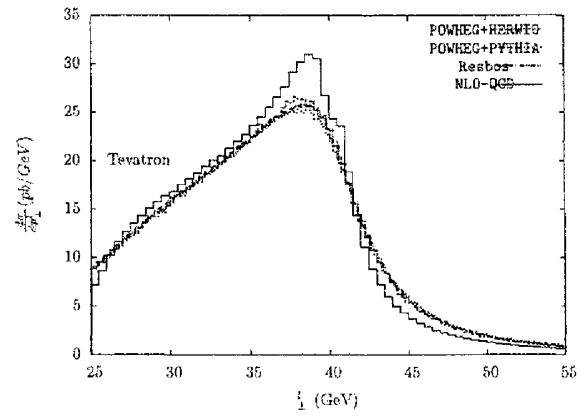

Figure 5: Same as in fig.4, for the transverse lepton distribution.

The templates used to evaluate the effects in fig. 4,5 have been prepared using Resbos, best approximation, $\alpha(0)$ input scheme, 1 billion of events, $80.348 \leq M W \leq 80.448 \mathrm{GeV}$.

The effect of the corrections is very stable in the case of the transverse mass distribution $(\triangle M W=+18 \mathrm{MeV})$ for both POWHEG+HERWOG and POWHEG+PYTHIA. In the case of the lepton transverse momentum distribution, the fitting procedure is much more sensitive to the QCD details of the different approximations, both for their impact on the normalization and on the shape of the distribution. 


\section{Effect of the combination of EW and QCD corrections}

The QCD and EW corrections to different distributions can be combined, up to terms of $\mathcal{O}\left(\alpha \alpha_{s}\right)$ and of terms of $\mathcal{O}\left(\alpha_{s}^{2}\right)$, according to the following recipes.

$$
\begin{gathered}
{\left[\frac{d \sigma}{d \mathcal{O}}\right]_{Q C D \notin E W}=\left\{\frac{d \sigma}{d \mathcal{O}}\right\}_{Q C D}+\left\{\left[\frac{d \sigma}{d \mathcal{O}}\right]_{E W}-\left[\frac{d \sigma}{d \mathcal{O}}\right]_{B O r n}\right\}} \\
{\left[\frac{d \sigma}{d \mathcal{O}}\right]_{Q C D \otimes E W}=\left(1+\frac{\left[\frac{d \sigma}{d \mathcal{O}}\right]_{M C @ N L O}-\left[\frac{d \sigma}{d \mathcal{O}}\right]_{H E R W I G P S}}{\left[\frac{d \sigma}{d \mathcal{O}}\right]_{L O / N L O}}\right) \times\left\{\left[\frac{d \sigma}{d \mathcal{O}}\right]_{E W}\right\}_{H E R W I G P S}}
\end{gathered}
$$

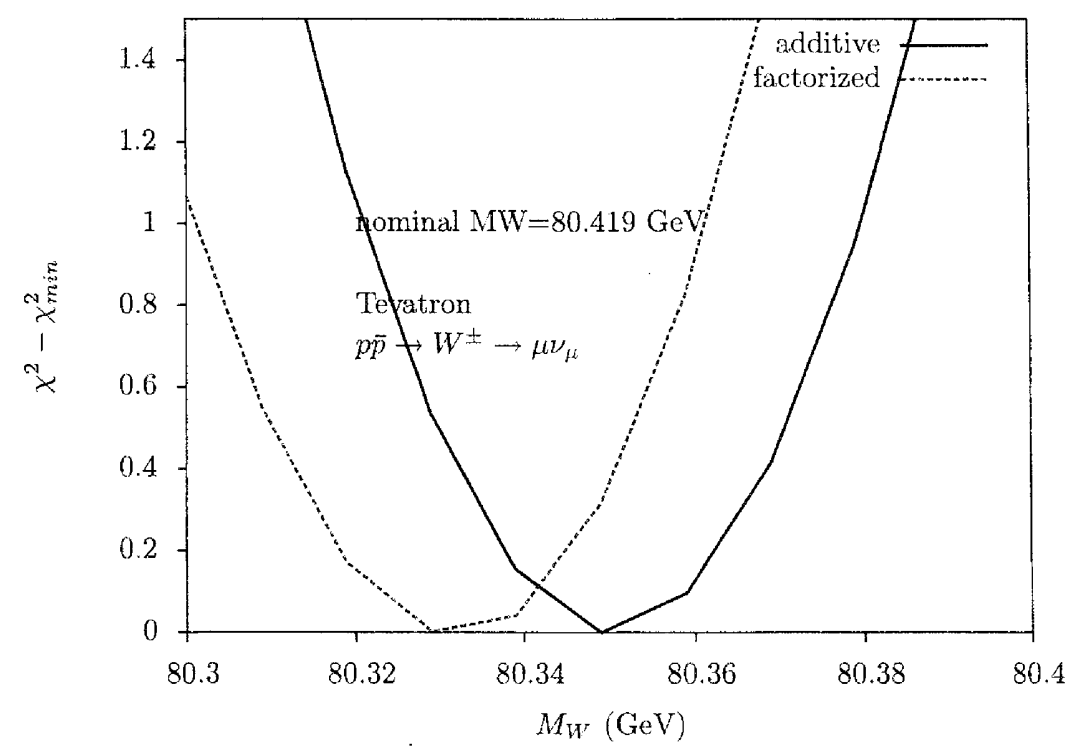

Figure 6: Preferred MW value obtained in the two approximations of eq.2 and of cq. 3

The templates used to evaluate the effects in fig. 6 have been prepared using Resbos, best approximation, $\alpha(0)$ input scheme, 1 billion of events, $80.219 \leq M W \leq 80.419 \mathrm{GeV}$, 


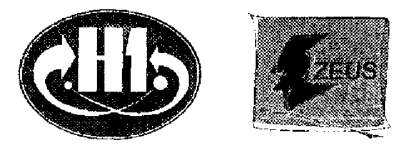

\title{
$W$ and $Z$ Physics from HERA
}

\section{David South (Technische Universität Dortmund)}

\author{
on behalf of the $\mathrm{H} 1$ and ZEUS Collaborations
}

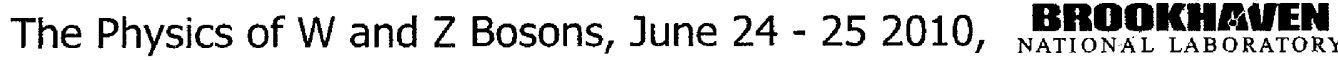

\begin{tabular}{|l|l|}
\hline & $\begin{array}{l}\text { Outline: } \\
\text { Introduction to HERA, H1 and ZEUS } \\
\text { High } \mathrm{Q}^{2} \text { Measurements of Neutral and Charge Current } \\
\text { Combined } \mathrm{H} 1 \text { and ZEUS measurements and QCD/EW fits } \\
\text { Rare processes at HERA involving W and Z Bosons } \\
\text { Summary }\end{array}$ \\
\hline
\end{tabular}




\section{Electroweak Physics at HERA}
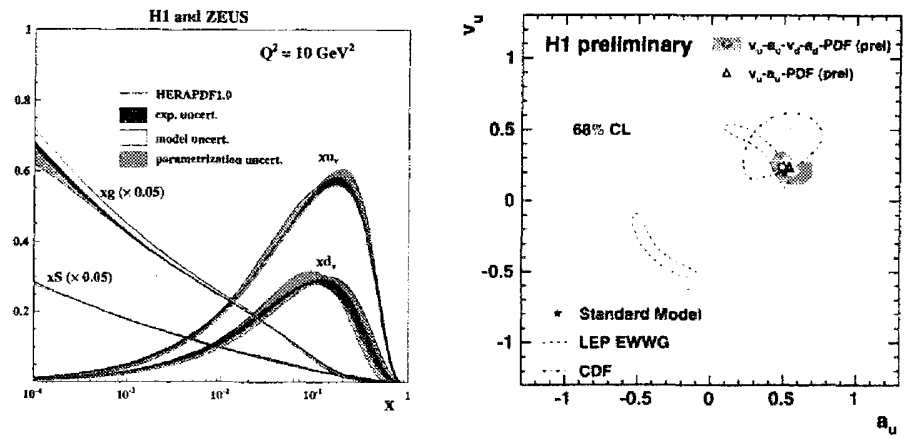

QCD and electroweak fits to the HERA data

Inclusive measurements electroweak effects at $\mathrm{Q}^{2} \sim \mathrm{M}_{\mathrm{W}, \mathrm{Z}}$
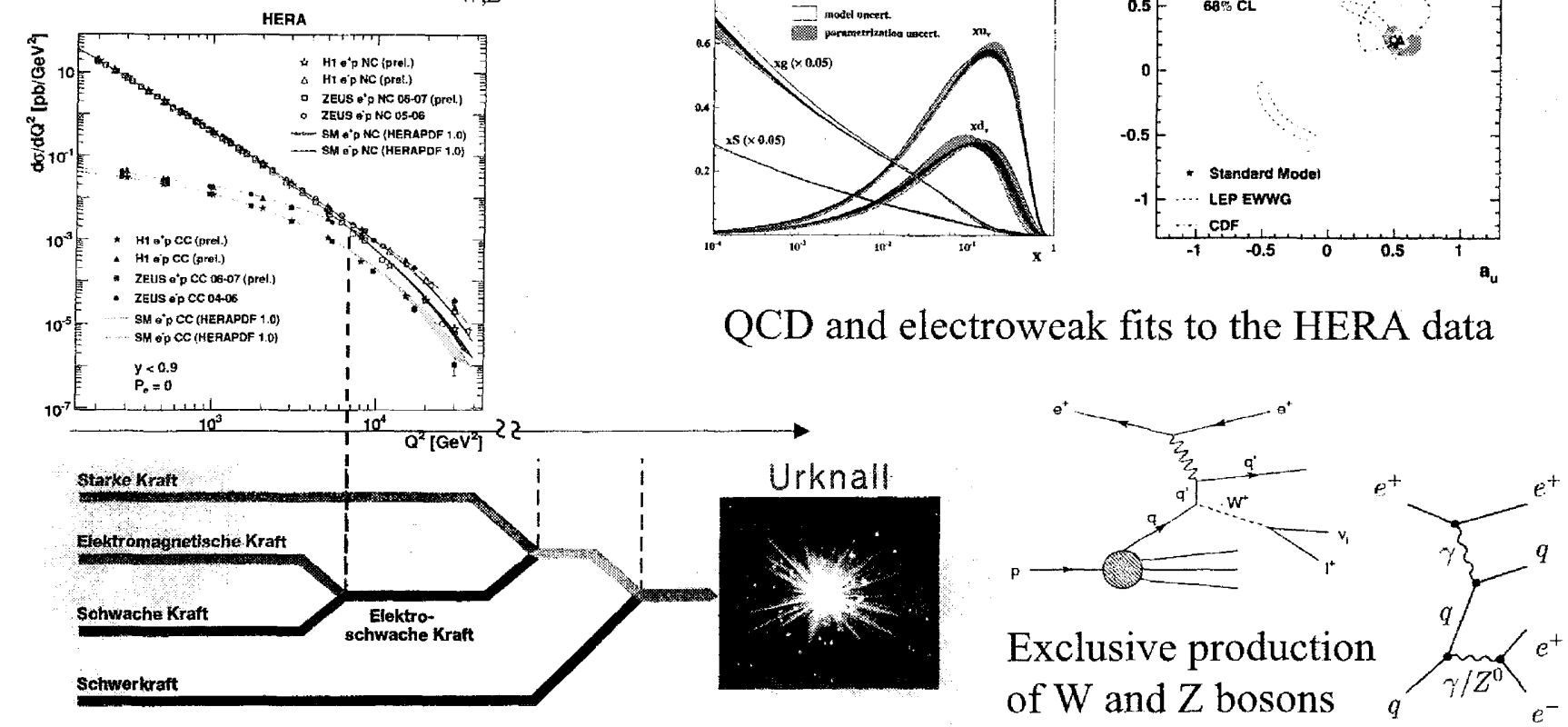

Measurements with $\mathrm{W}$ and $\mathrm{Z}$ bosons at HERA are within reach! 


\section{HERA II Polarisation Asymmetry in NC}

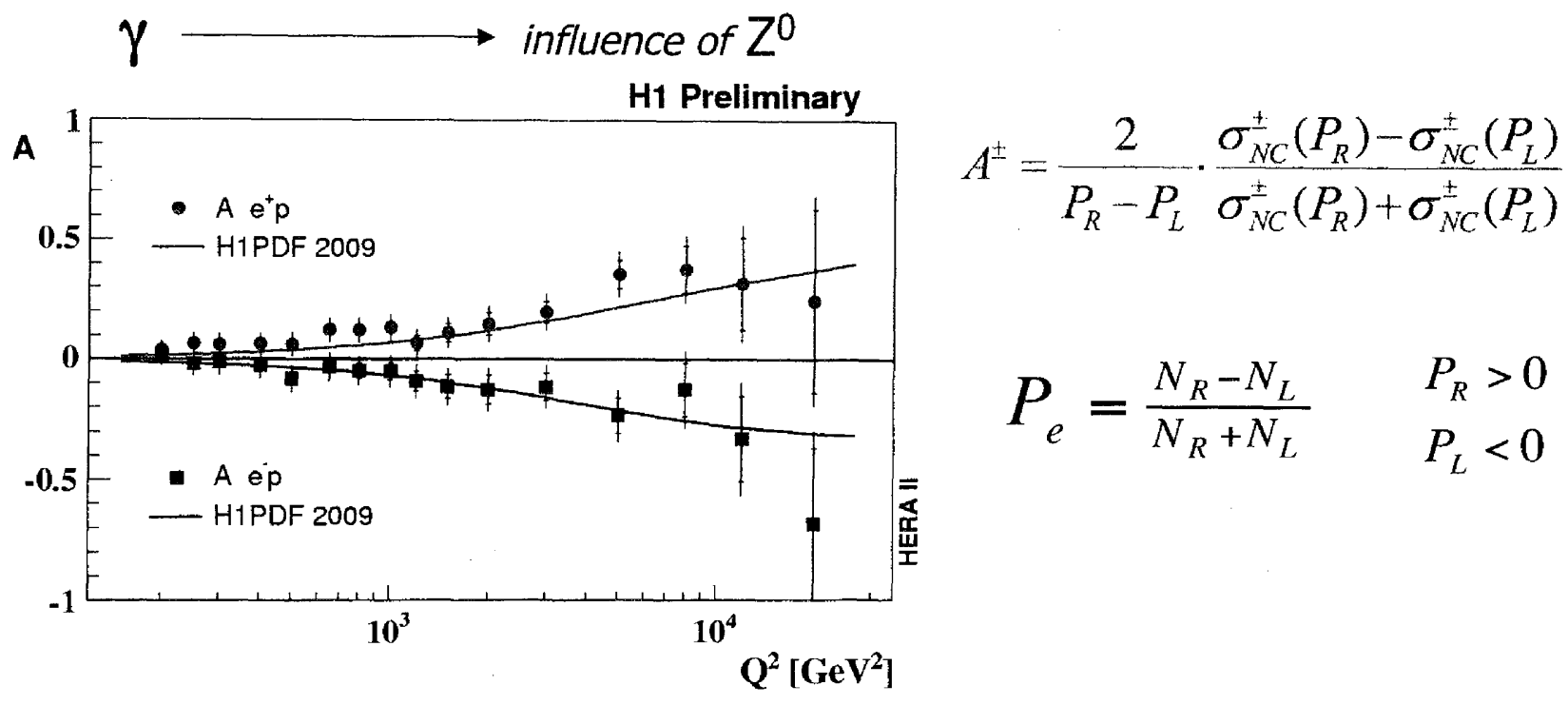

Form polarisation asymmetry from HERA II Neutral Current measurements

- clear observation of parity violation of NC electroweak exchange

Nicely illustrates the properties of the different polarisation and lepton charge data

Well described by the SM prediction 


\section{Charged Current Cross Section vs. Polarisation}
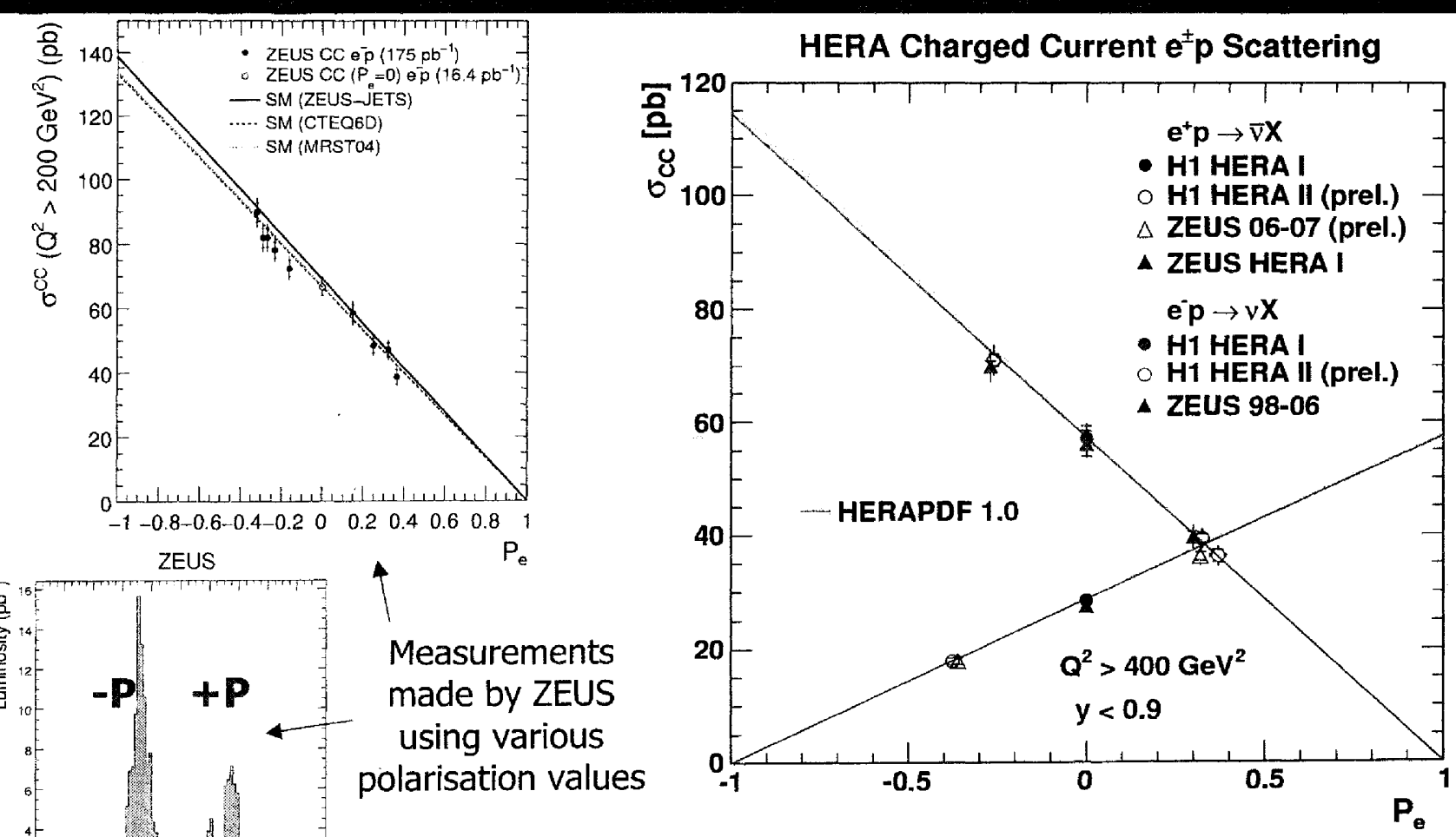

Data exhibit linear dependence of average polarisation and HERA I and II measurements agree with the SM prediction 


\section{Impact of HERA Data at the LHC}

$\mathrm{W}^{+}, \mathrm{Z}$ rapidities (at $14 \mathrm{TeV}$ !)

Prior to any HERA data
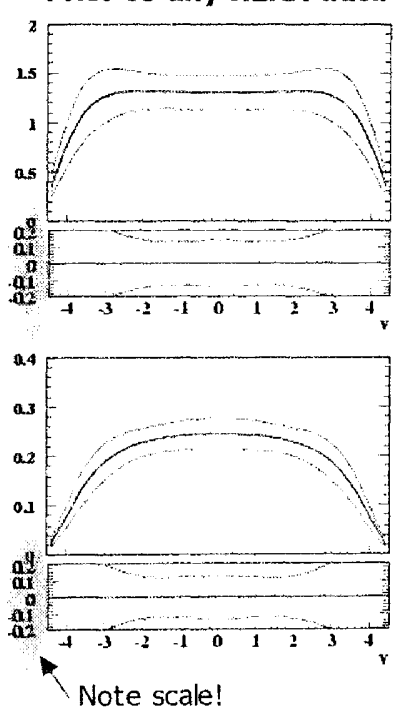

Note scale!
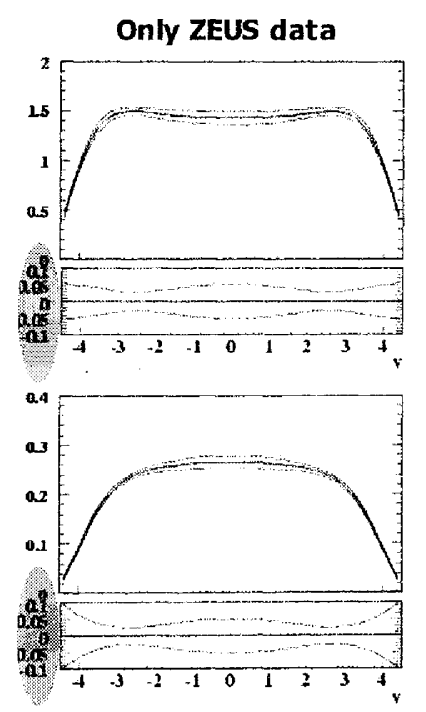

Experimental uncertainty at central rapidities using combined HERA data $1 \%$ http://www.desy.de/h1zeus/combined_results/benchmark/herapdf1.0.html

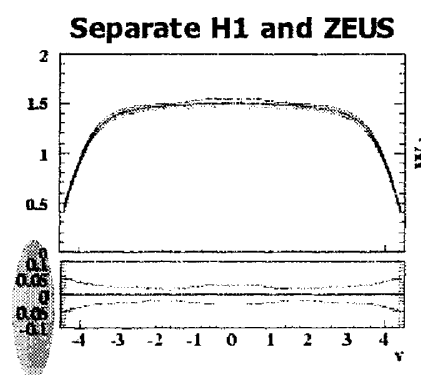

HERAPDF 1.0
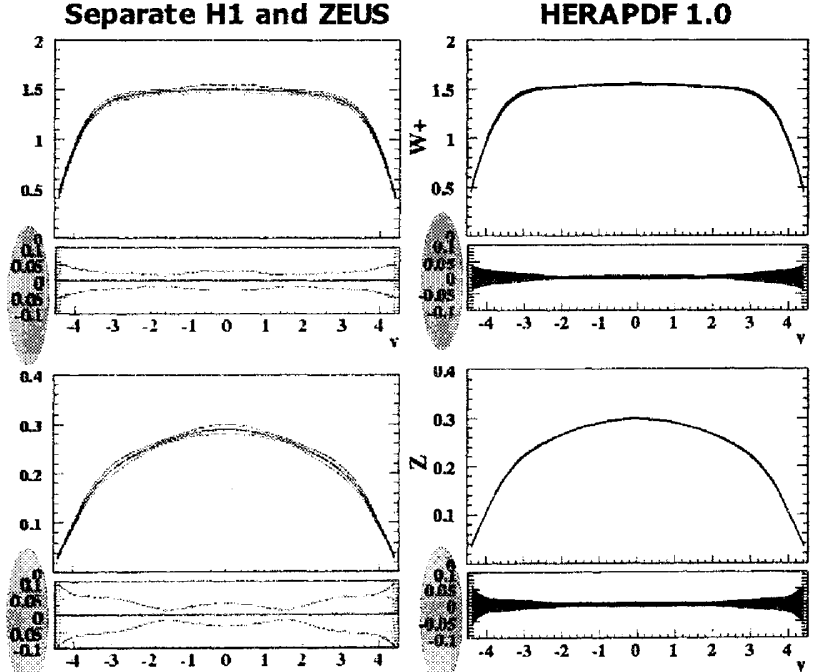

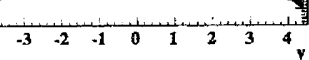

ed HERA data $1 \%$

after Voica Radescu, and Amanda Cooper-Sarkar

Impressive precision on the low $x$ sea and gluon of the HERAPDF 1.0 is relevant for $W, Z$ production at the LHC

Inclusion of HERA data shows the tremendous improvement on the predictions for $\mathrm{W}$ and $\mathrm{Z}$ production at the central rapidity 


\section{H1+ZEUS Isolated Leptons: Results}
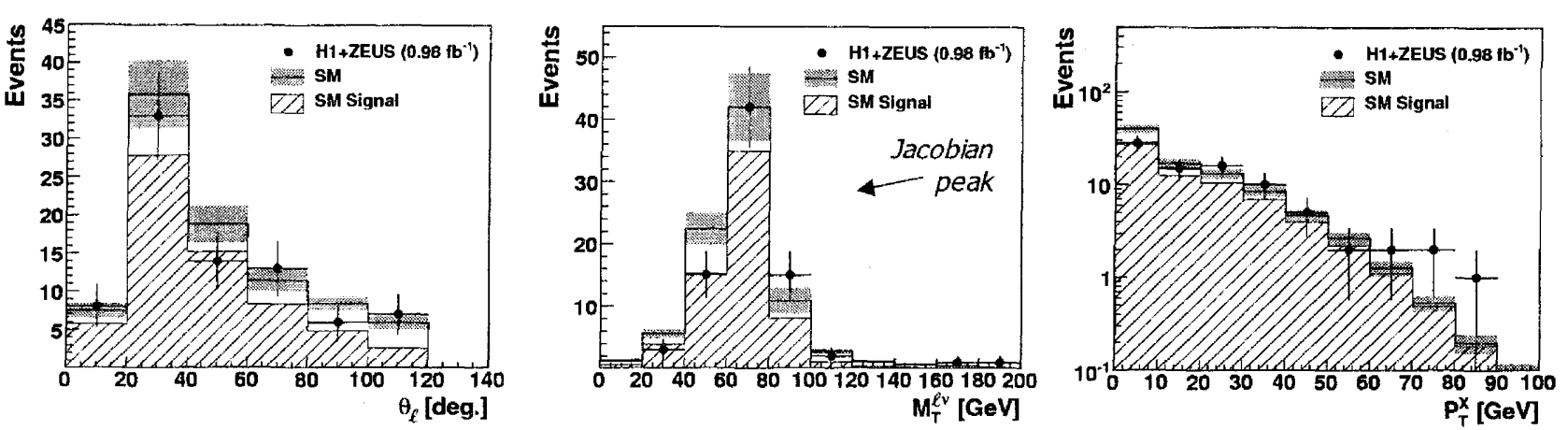

\begin{tabular}{|c|c|c|c|c|c|c|c|c|c|}
\hline \multicolumn{2}{|c|}{$\begin{array}{l}\text { H1+ZEUS } \\
1994-2007 e^{ \pm} p\end{array}$} & $0.98 \mathrm{fb}^{-1}$ & Data & \multicolumn{3}{|c|}{$\begin{array}{c}\text { SM } \\
\text { Expectation }\end{array}$} & $\begin{array}{c}\text { SM } \\
\text { Signal }\end{array}$ & $\begin{array}{l}\text { Other SM } \\
\text { Processes }\end{array}$ & \multirow{3}{*}{$\begin{array}{l}\text { Good overall } \\
\text { agreement } \\
\text { with the } \\
\text { Standard Model }\end{array}$} \\
\hline \multirow[t]{2}{*}{ Electron } & & Total & 61 & & & & $48.3 \pm 7.4$ & $20.9 \pm 3.2$ & \\
\hline & & $>20$ Ger & 16 & 10.0 & & 1.7 & $10.0 \pm 1.6$ & $3.1 \pm 0.7$ & \\
\hline \multirow[t]{2}{*}{ Muon } & & & & & & 2.7 & $16.4 \pm 2.6$ & $2.2 \pm 0.5$ & \multirow{4}{*}{$\begin{array}{l}\text { SM expectation } \\
\text { dominated W } \\
\text { production } \\
\rightarrow \text { Cross section }\end{array}$} \\
\hline & & $>25 \mathrm{GeV}$ & 13 & 11.0 & & 1.6 & $\pm \quad 1.6$ & $1.2 \pm 0.3$ & \\
\hline \multirow[t]{2}{*}{ Combined } & & Total & 81 & 87.8 & \pm & & $64.7 \pm 9.9$ & $23.1 \pm 3.3$ & \\
\hline & & $>25 \mathrm{GeV}$ & 29 & 24.0 & & 3.2 & \pm 3.1 & $4.3 \pm 0.8$ & \\
\hline
\end{tabular}

Measured: $1.06 \pm 0.16$ (stat.) \pm 0.07 (sys.) pb SM: $1.26 \pm 0.19 \mathrm{pb}$ from EPVEC NLO

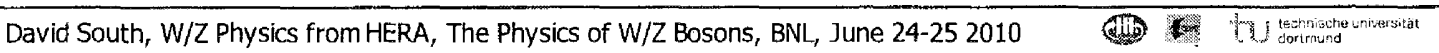




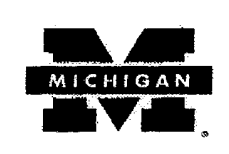

Dø

\title{
Measurement of $\mathrm{W}$ boson mass at $\mathrm{D} \varnothing$
}

\author{
Junjie Zhu \\ University of Michigan
}

The Physics of $\mathrm{W}$ and $\mathrm{Z}$ Bosons

June 24, 2010 


\section{W boson mass}

$$
M_{W}^{2}=\frac{\pi \alpha}{\sqrt{2} G_{F}} \frac{1}{\sin ^{2} \theta_{W}} \frac{1}{(1-(\Delta \nabla))} \quad \Delta r \sim \mathbf{3} \%
$$

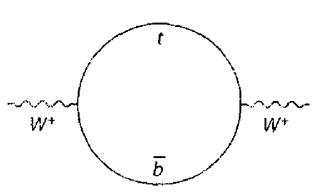

$\Delta r \propto \mathrm{M}_{\mathrm{t}}^{2}$

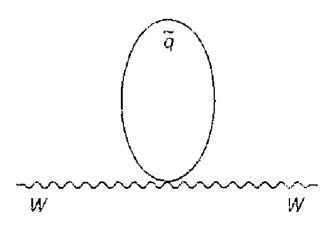

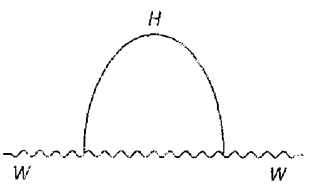

$\Delta r \propto \log \mathrm{M}_{\mathrm{H}}$

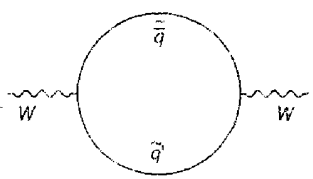

$\mathrm{M}_{\mathrm{W}}$ can be increased by up to $250 \mathrm{MeV}$ in MSSM

A precise measurement of $\mathrm{M}_{\mathrm{W}}$ can be used to make indirect constraints on $\mathrm{M}_{\mathrm{H}}$ and possible new physics 


\section{Measurement strategy}
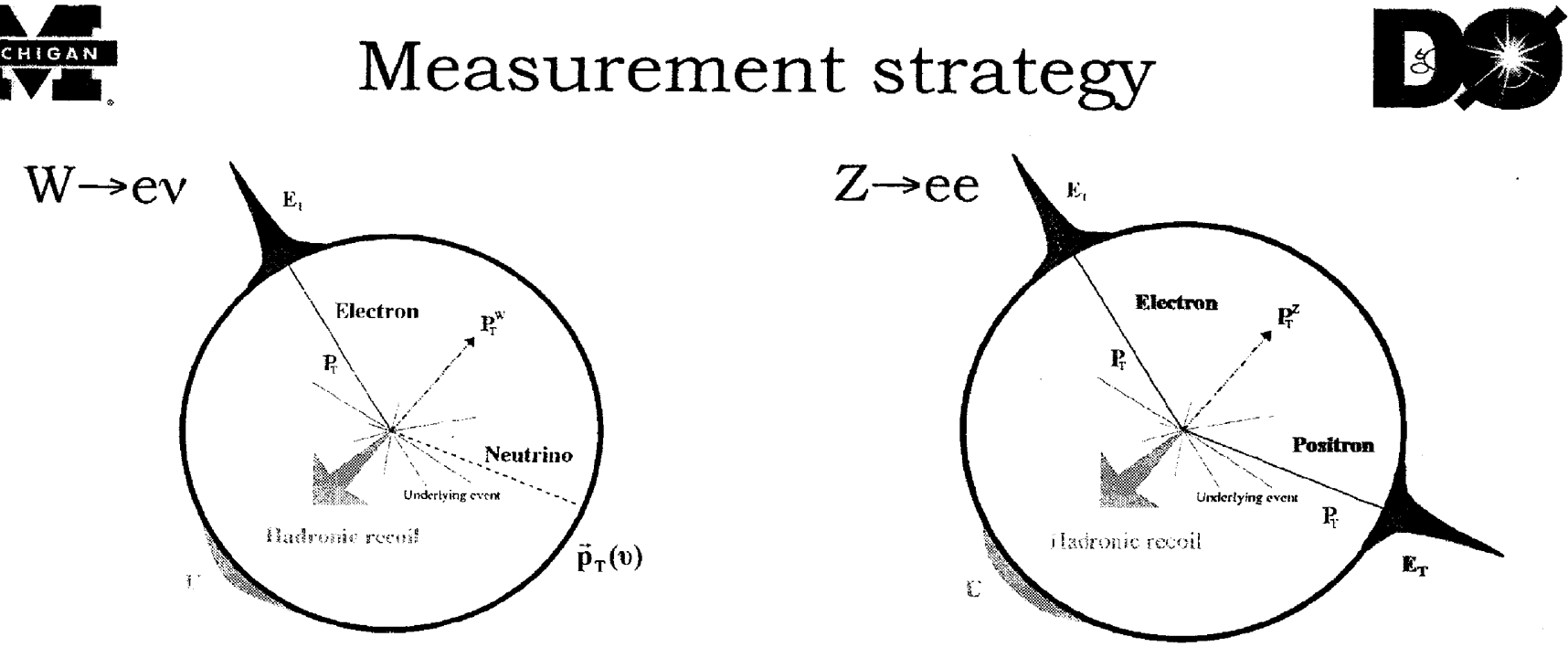

- Three observables: $\mathrm{p}_{\mathrm{T}}(\mathrm{e}), \mathrm{p}_{\mathrm{T}}(v)$ (inferred from missing transverse energy), transverse mass $\mathrm{M}_{\mathrm{T}}{ }^{2}=\left(\mathrm{E}_{\mathrm{Te}}+\mathrm{E}_{\mathrm{T} v}\right)^{2}-\left|\overrightarrow{\mathrm{p}}_{\mathrm{Te}}+\overrightarrow{\mathrm{p}}_{\mathrm{T} v}\right|^{2}$

- Develop a parameterized MC simulation with parameters determined from the collider data (mainly $Z \rightarrow$ ee events)

- Generate MC templates with different input $\mathrm{M}_{\mathrm{W}}$, compare with data distributions and extract $M_{W}$

- $Z \rightarrow$ ee events are used to set the absolute electron energy scale, so we are effectively measuring $\mathrm{M}_{\mathrm{W}} / \mathrm{M}_{Z}$ 


\section{Calibration results}
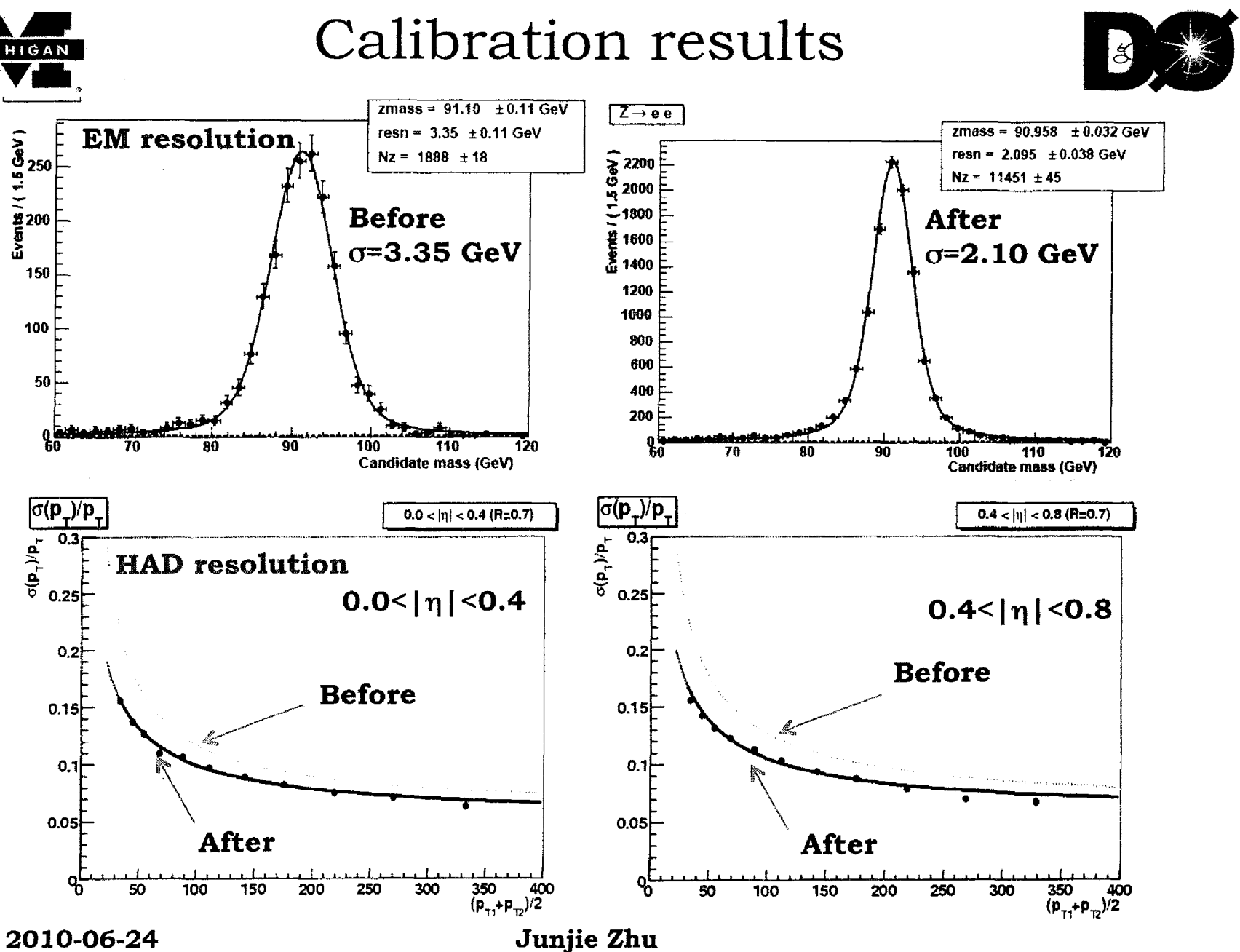

2010-06-24

Junjie Zhu 


\section{Mass fits}

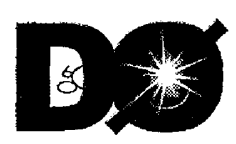

$Z$ invariant mass $\left(M_{e e}\right), 18 k$

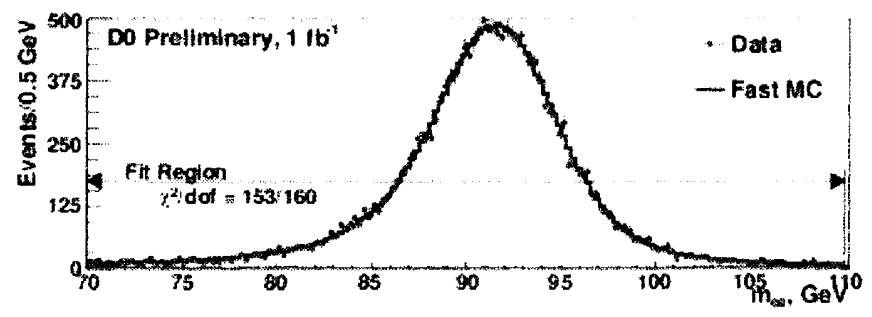

W transverse mass $\left(M_{T}\right), 500 k$
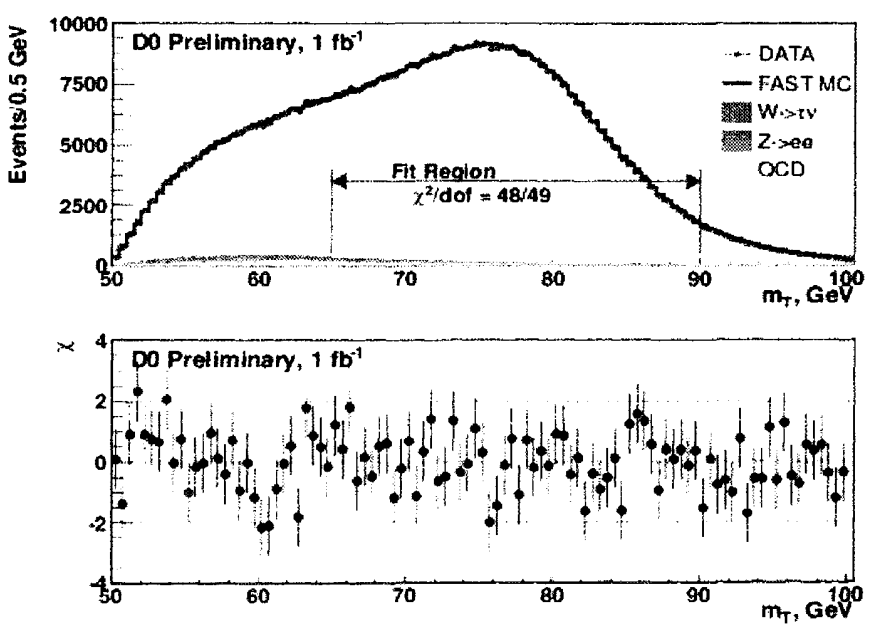

$M_{w}=80.401 \pm 0.023$ (stat) $\mathrm{GeV}$

(WA $M_{z}=91.188 \pm 0.002 \mathrm{GeV}$ )

PRL 103, 141801 (2009)

2010-06-24

Junjie Zhu 


\section{Uncertainties}

$=$ $\sigma\left(m_{W}\right) \mathrm{MeV}$

Source $\quad m_{T} \quad p_{T}^{e} \quad \not_{T}$

Electron energy calibration

Electron resolution model

Electron energy offset

Electron energy loss model

Recoil model

Electron efficiencies

Backgrounds

\begin{tabular}{|ccc|}
\hline 34 & 34 & 34 \\
\hline 2 & 2 & 3
\end{tabular}

Experimental Subtotal

PDF

QED

Boson $p_{T}$

$4 \quad 6 \quad 7$

Production Subtotal

\begin{tabular}{|ccc|}
4 & 4 & 4 \\
\hline 6 & 12 & 20 \\
\hline
\end{tabular}

Total Systematic

Statistical

$\begin{array}{lll}5 & 6 & 5\end{array}$

Total

\begin{tabular}{|ccc|}
\hline 35 & 37 & 41 \\
\hline 9 & 11 & 14 \\
\hline 7 & 7 & 9 \\
\hline 2 & 5 & 2 \\
\hline 12 & 14 & 17 \\
\hline 37 & 40 & 44 \\
\hline 23 & 27 & 23 \\
\hline 44 & 48 & 50 \\
\hline
\end{tabular}

2010-06-24

Junjie Zhu 


\section{Measurement of the W Boson Mass at CDF \\ Ashutosh Kotwal \\ Duke University}

We present a techniques used for precise measurements of the $\mathrm{W}$ boson mass at the CDF experiment at Fermilab. We present the results and the prospects for future improvements at Fermilab and the LHC. 


\section{Outline of CDF Analysis}

Energy scale measurements drive the $W$ mass measurement

- Tracker Calibration

- alignment of the central drift chamber (COT with $\sim 2400$ cells) using cosmic rays

- COT momentum scale and tracker non-linearity constrained using $\mathrm{J} / \Psi \rightarrow \mu \mu$ and $\gamma \rightarrow \mu \mu$ mass fits

- Confirmed using $\mathrm{Z} \rightarrow \mu \mu$ mass fit

- EM Calorimeter Calibration

- COT momentum scale transferred to EM calorimeter using a fit to the peak of the $\mathrm{E} / \mathrm{p}$ spectrum, around $\mathrm{E} / \mathrm{p} \sim 1$

- Calorimeter energy scale confirmed using $Z \rightarrow$ ee mass fit

- Tracker and EM Calorimeter resolutions

- Hadronic recoil modelling

- Characterized using $\mathrm{p}_{\mathrm{T}}$-balance in $\mathrm{Z} \rightarrow l l$ events 


\section{Internal Alignment of COT}

- Use a clean sample of $\sim 200 k$ cosmic rays for cell-by-cell internal alignment

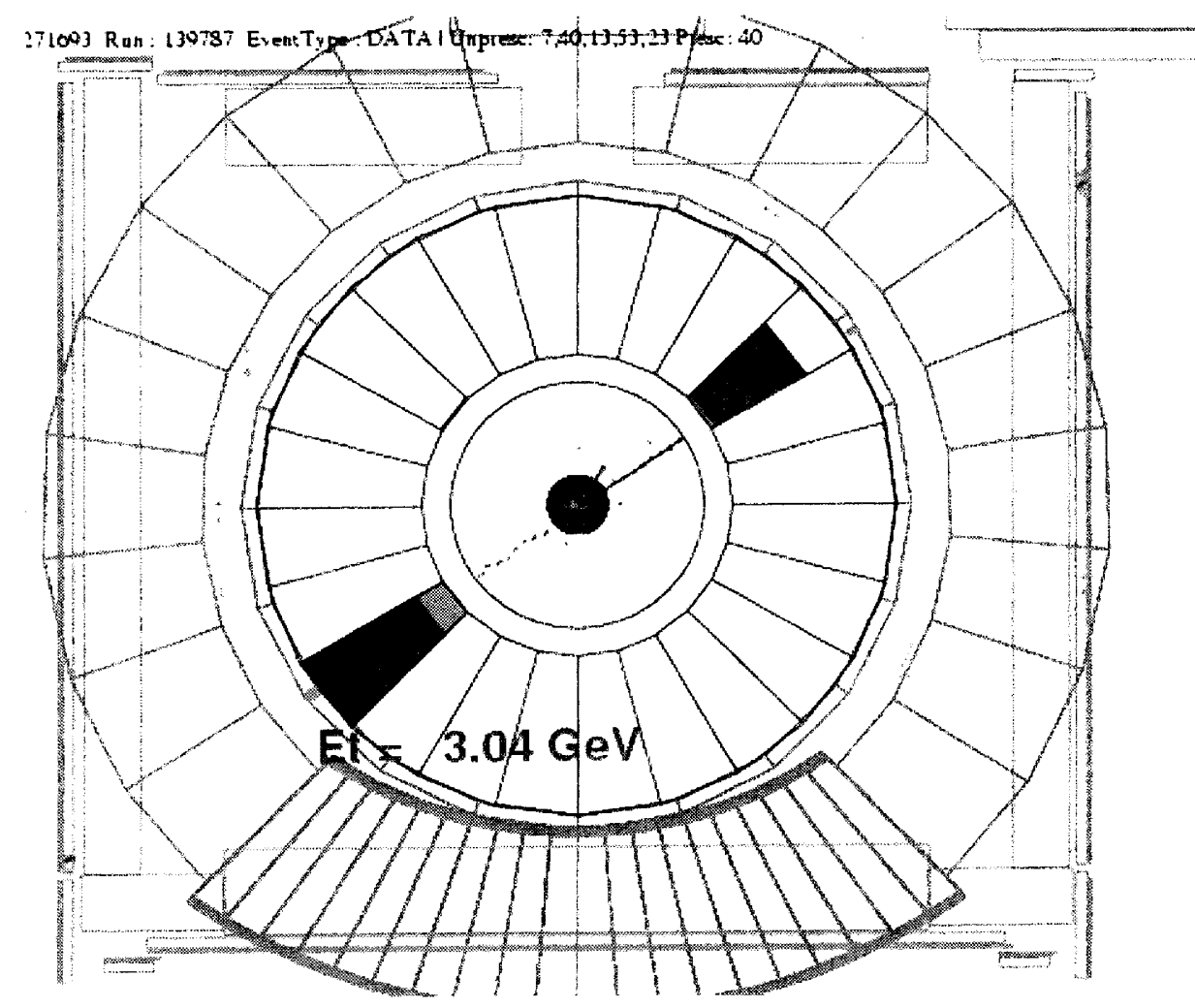

- Fit COT hits on both sides simultaneously to a single helix (A.Kotwal, H. Gerberich and C. Hays, NIMA 506, $110(2003))$

- Time of incidence is a floated parameter

- Same technique being used on ATLAS and CMS 


\section{Tracking Momentum Calibration}

- Set using $\mathrm{J} / \Psi \rightarrow \mu \mu$ and $\Upsilon \rightarrow \mu \mu$ resonances

- Consistent within total uncertainties

- Use $\mathrm{J} / \Psi$ to study and calibrate non-linear response of tracker

- Systematics-dominated, improved detector modelling required
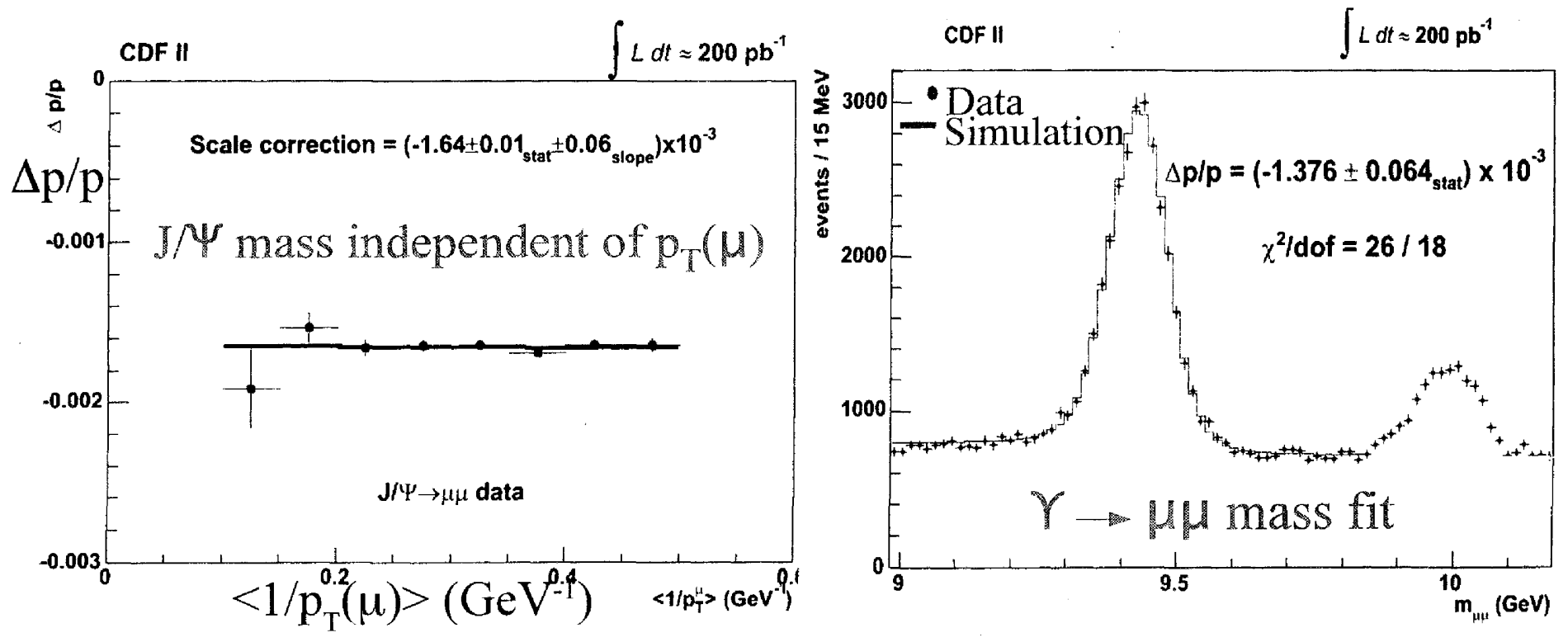


\section{$Z \rightarrow l l$ Mass Cross-checks}

- $\mathrm{Z}$ boson mass fits consistent with tracking and $\mathrm{E} / \mathrm{p}$-based calibrations

CDF II

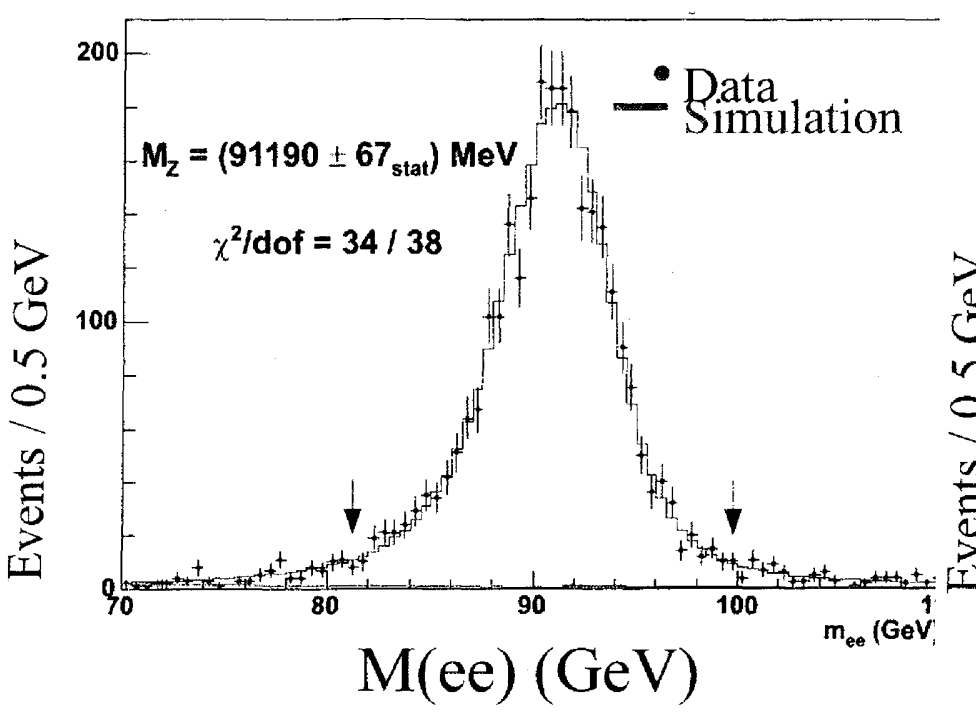

$L \sim 200 / \mathrm{pb}$

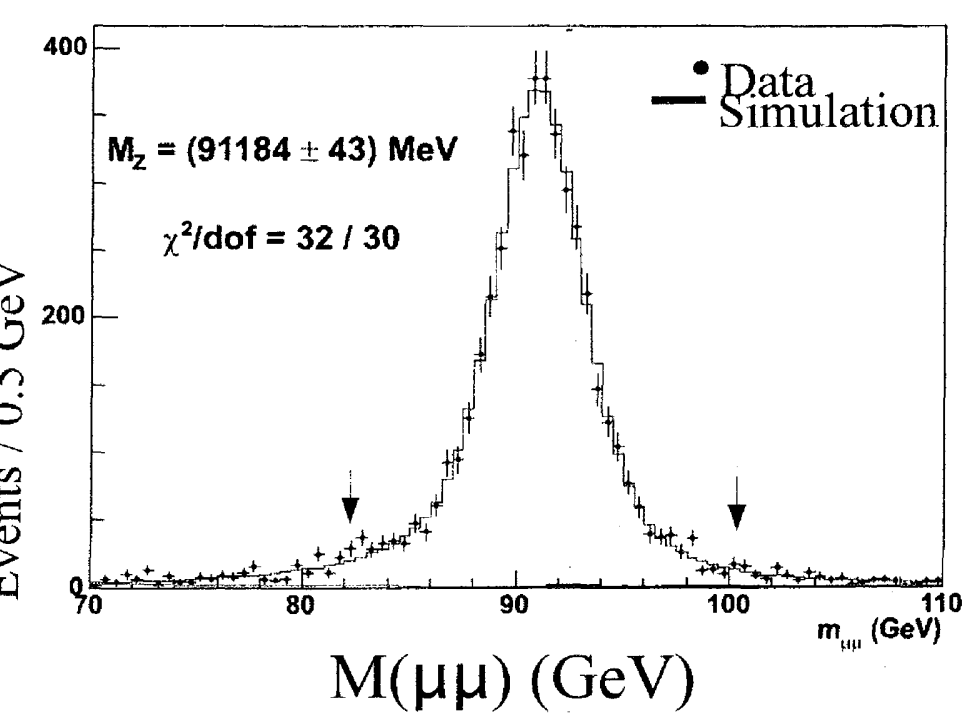




\section{Summary}

- The $W$ boson mass is a very interesting parameter to measure with increasing precision

- CDF Run $2 \mathrm{~W}$ mass result with $200 \mathrm{pb}^{-1}$ data:

$-\mathrm{M}_{\mathrm{W}}=80413 \pm 48 \mathrm{MeV}$

- D0 Run $2 \mathrm{~W}$ mass result with $1 \mathrm{fb}^{-1}$ data:

A

- $\mathrm{M}_{\mathrm{W}}=80401 \pm 43 \mathrm{MeV}$

- Most systematics limited by statistics of control samples

- CDF and D0 are both working on $\delta \mathrm{M}_{\mathrm{W}}<25 \mathrm{MeV}$ measurements from $\sim 2 \mathrm{fb}^{-1}(\mathrm{CDF})$ and $\sim 4 \mathrm{fb}^{-1}(\mathrm{D} 0)$

- Learning as we go: Tevatron $\rightarrow$ LHC may produce $\delta \mathrm{M}_{\mathrm{W}} \sim 5-10 \mathrm{MeV}$ 


\title{
Monte Carlo modelling issues for $\mathrm{W}$ measurements
}

\author{
Jan Stark \\ Laboratoire de Physique Subatomique et de Cosmologie \\ Grenoble, France
}

The physics of $W$ and $Z$ bosons

RIKEN BNL Research Centre Workshop, June 24-25, 2010
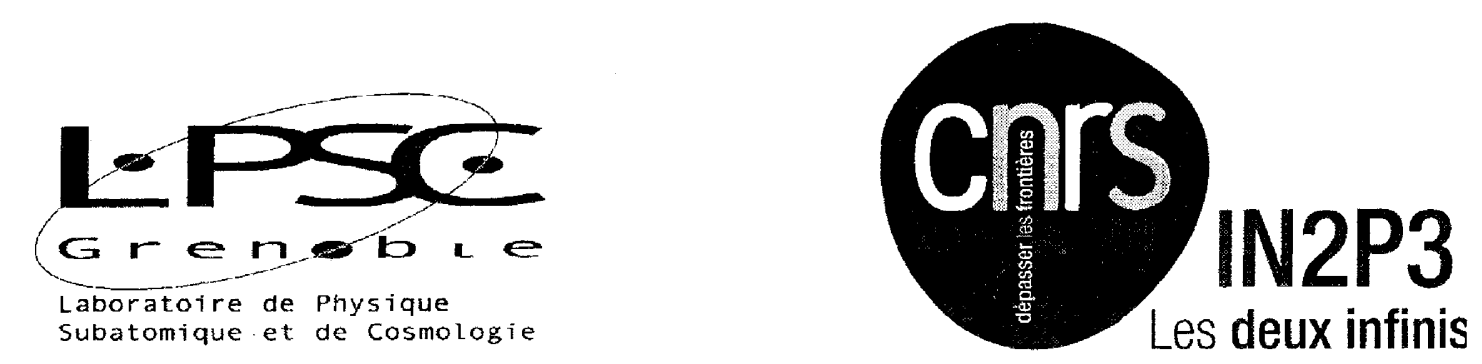


\section{Context}

Most of the comments in this talk are based on experience from the $D \varnothing 1 \mathrm{fb}^{-1}$ measurement of the $\mathrm{W}$ boson mass. A complete overview of this analysis was described in Junjie Zhu's talk earlier today. Here we provide more details on the use of simulations in this measurement.

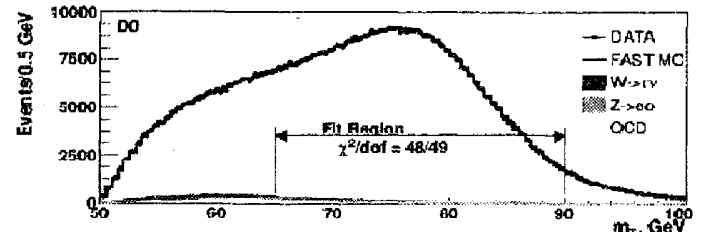

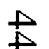
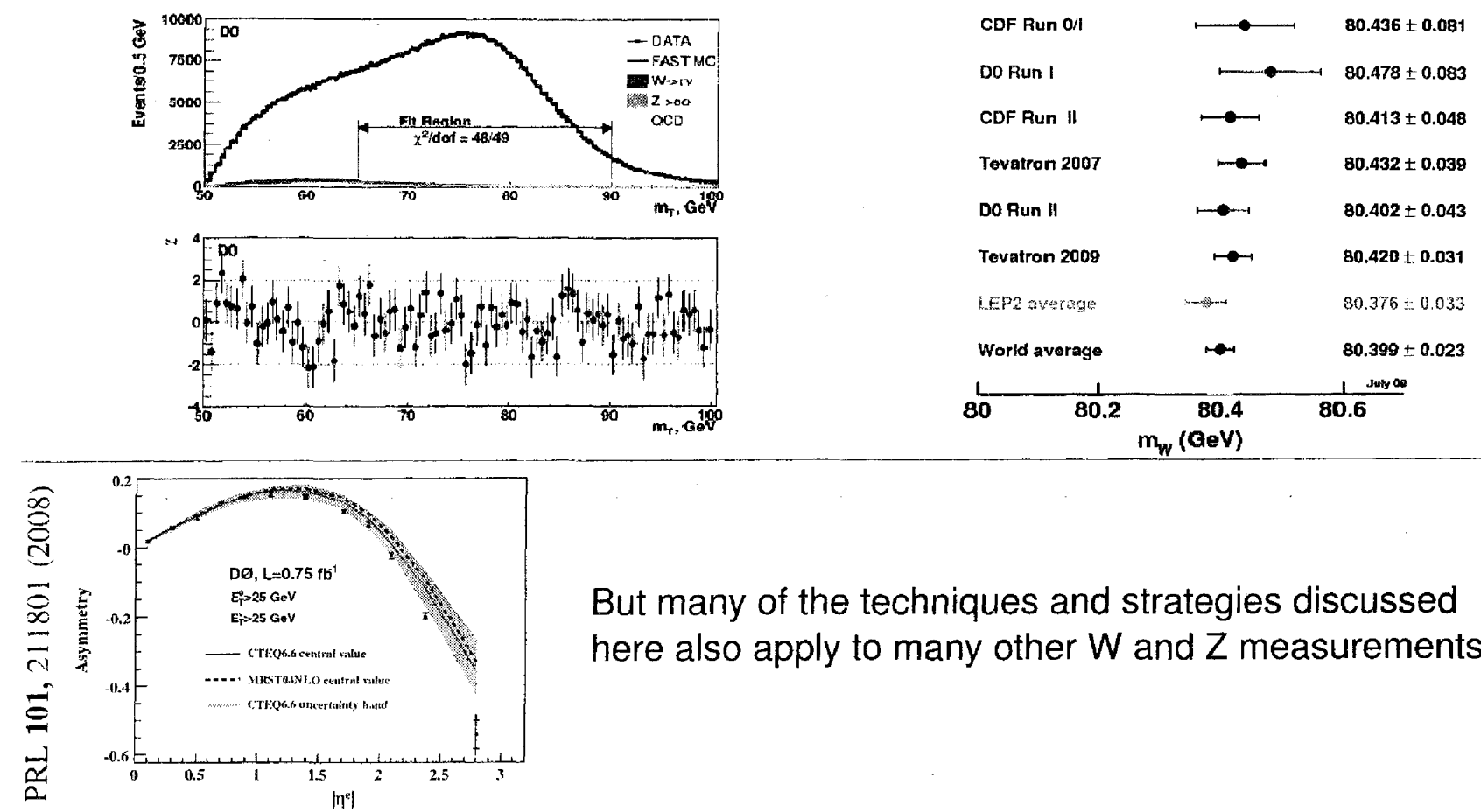

But many of the techniques and strategies discussed here also apply to many other $\mathrm{W}$ and $\mathrm{Z}$ measurements ...

Jan Stark

The physics of $W$ and $Z$ bosons, Brookhaven, June 24-25, 2010 


\section{Reminder: signature in the detector, requirements on precision}

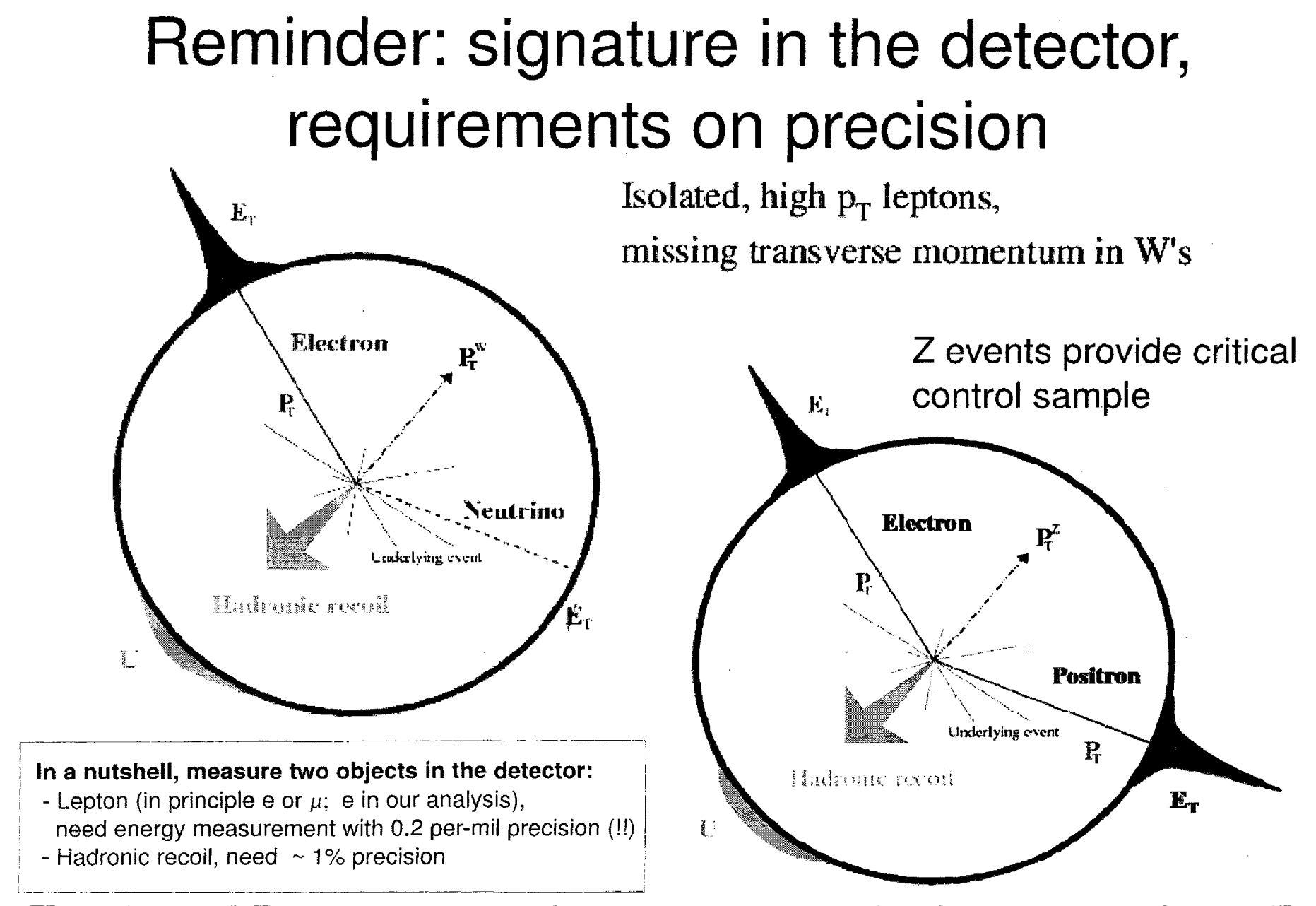




\section{Measurement strategy}

W mass is extracted from transverse mass, transverse momentum and transverse missing momentum:

Need Monte Carlo simulation to predict shapes of these observables for given mass hypothesis

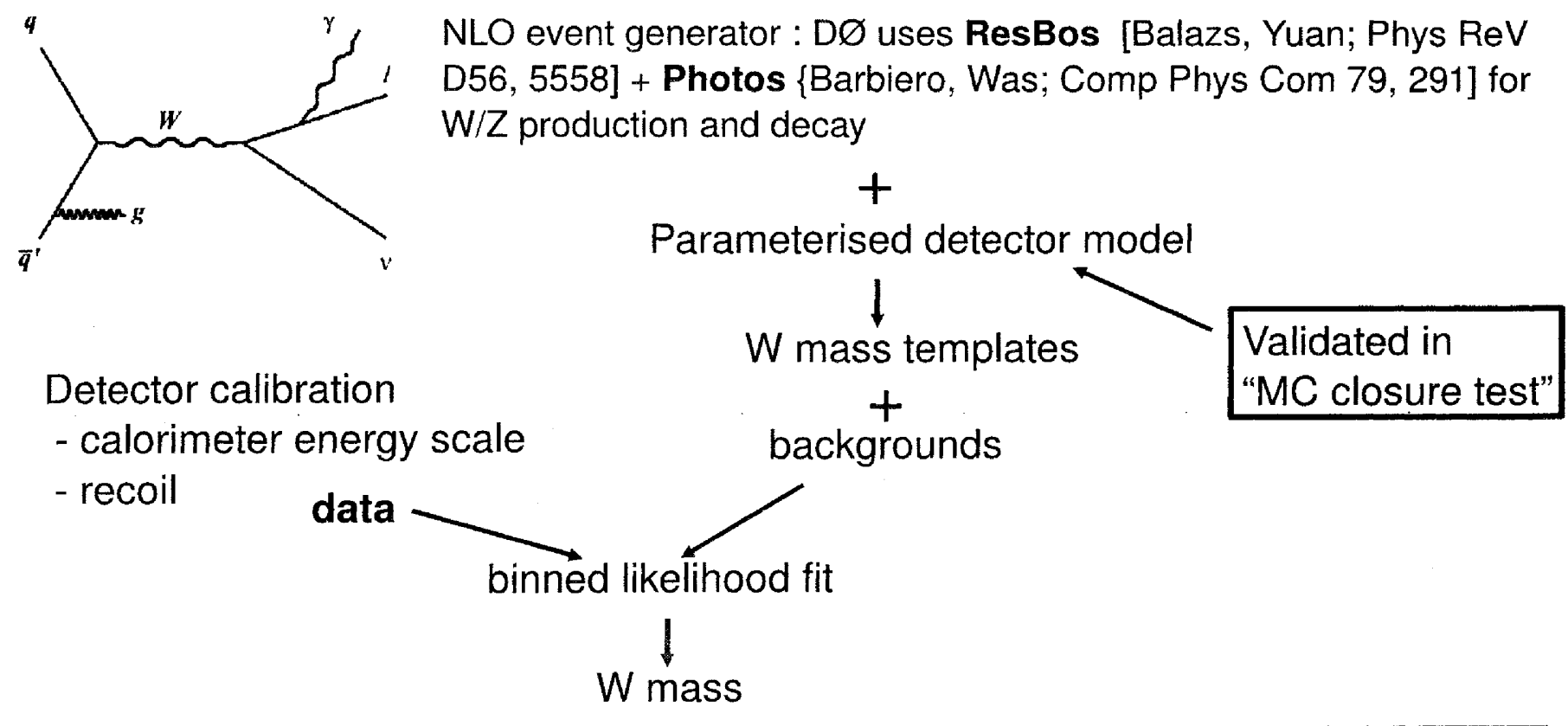




\section{"First principles" vs. "parameterised" simulations}

We all like "first principles" simulations, i.e. simulations where everything is based on a formal theory that predicts everything.

Examples: $\quad$ - A gauge theory used to simulate some $e^{+} e^{-} \rightarrow X$ collision.

- A simulation based on the known laws of the interactions between high-energy particles and matter, as well as a model of the $D \varnothing$ detector geometry is used to predict the electron energy response in $D \varnothing$.

But what to do when the "first principles" cannot be made precise/complete enough ?

Examples: - Tricky mathematical issues in QCD description of $\mathrm{p}^{+} \mathrm{p}^{+/-} \rightarrow \mathrm{X}$.

- Response to hadrons not simulated quite right in detector simulation.

$-\ldots$

Here "parameterised" simulations can be very powerful, because they have simple "knobs" that we can turn to adjust things.

Examples: - Non-perturbative form factors to be determined from collider data.

- Simple parameterisation of hadron energy response, to be fit to control sample from collider data.

In practice, the trick is to combine the two approaches. In the $D \varnothing \mathrm{m}(\mathrm{W})$ measurement we have a parameterised simulation with many parameterisations derived from first-principles simulations. 


\section{Model of W production and decay}

\begin{tabular}{c|ccl} 
Tool & Process & QCD & EW \\
\hline RESBOS & $W, Z$ & NLO & - \\
\hline WGRAD & $W$ & LO & complete $\mathcal{O}(\alpha)$, Matrix Element $\leq 1$ photon \\
ZGRAD & $Z$ & LO & complete $\mathcal{O}(\alpha)$, Matrix Element: $\leq 1$ photon \\
\hline PHOTOS & & & QED FSR $\leq 2$ photons
\end{tabular}

Our main generator is "ResBos+Photos". The NLO QCD in ResBos allows us to get a reasonable description of the $\mathrm{p}_{\mathrm{T}}$ of the vector bosons. The two leading EWK effects are the first FSR photon and the second FSR photon. Photos gives us a reasonable model for both.

We use W/ZGRAD to get a feeling for the effect of the full EWK corrections.

The final "QED" uncertainty we quote is $7 / 7 / 9 \mathrm{MeV}\left(\mathrm{m}_{\mathrm{T}}, \mathrm{p}_{\mathrm{T}}, \mathrm{MET}\right)$. This is the sum of different effects; the two main ones are:

- Effect of full EWK corrections, from comparison of W/ZGRAD in "FSR only" and in "full EWK" modes (5/5/5 MeV).

- Very simple estimate of "quality of FSR model", from comparison of W/ZGRAD in FSR-only mode vs Photos (5/5/5 MeV).

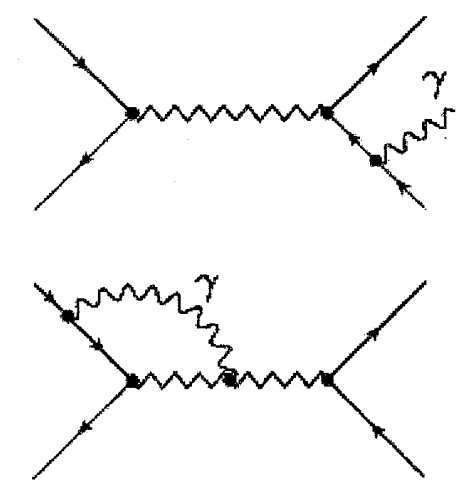




\section{Theoretical issues in Monte Carlo modelling for $W / Z$ production}

[ Physics of W and Z bosons - workshop @ RIKEN BNL research center ]

$$
\begin{gathered}
\text { Jan Winter }{ }^{a} \\
\text { - Fermilab - }
\end{gathered}
$$

I give an overview of how vector boson production is

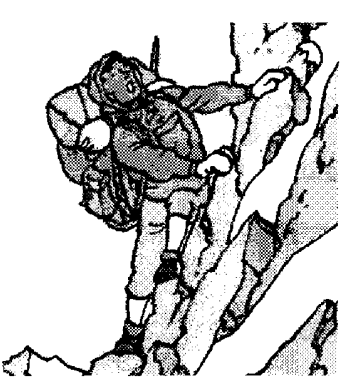
processed in Monte Carlo event generators. In a high-

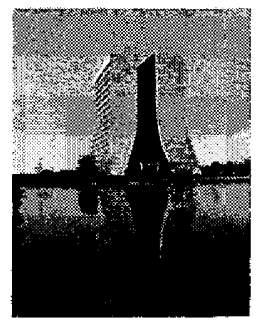
energy hadron collider environment this is always affected by $Q C D$ radiation. Parton showers can capture the leading effects of soft and collinear emissions, but fail to sufficiently describe hard jets associated with the vector boson. One therefore has to improve parton-shower approaches to gain a good understanding of $\mathrm{V}+\mathrm{n}$ jets - a major background to all new physics searches. I briefly review tree-level matrix-element plus partonshower merging and NLO calculations as means to predict $V+n$ jet production. I compare both types of calculations and discuss their results.

\footnotetext{
${ }^{a}$ Sherpa authors: J. Archibald, T. Gleisberg, S. Höche, H. Hoeth, F. Krauss, M. Schönherr, F. Siegert,
}

S. Schumann, J. Winter and K. Zapp

http://www.sherpa-mc.de/ 


\section{Monte Carlo modelling of a (high- $p_{T}$ ) event}

$\rightarrow$ Factorization approach: divide jet simulation into different phases

$\rightarrow$ Perturbative Phases: [parton jets]

\& Hard process/interaction (hard jet production) exact matrix elements $|\mathcal{M}|^{2}$

- QCD bremsstrahlung (soft/coll multiple emissions) initial- and final-state parton showering

c Multiple/Secondary interactions modelling the underlying event

$\rightarrow$ Non-perturbative Phases: [jet confinement-particle jets]

4 Hadronizañon phenomenological models to convert partons into primary hadrons

- Hadron decays phase-space or effective models to decay unstable into stable hadrons as observed in detectors

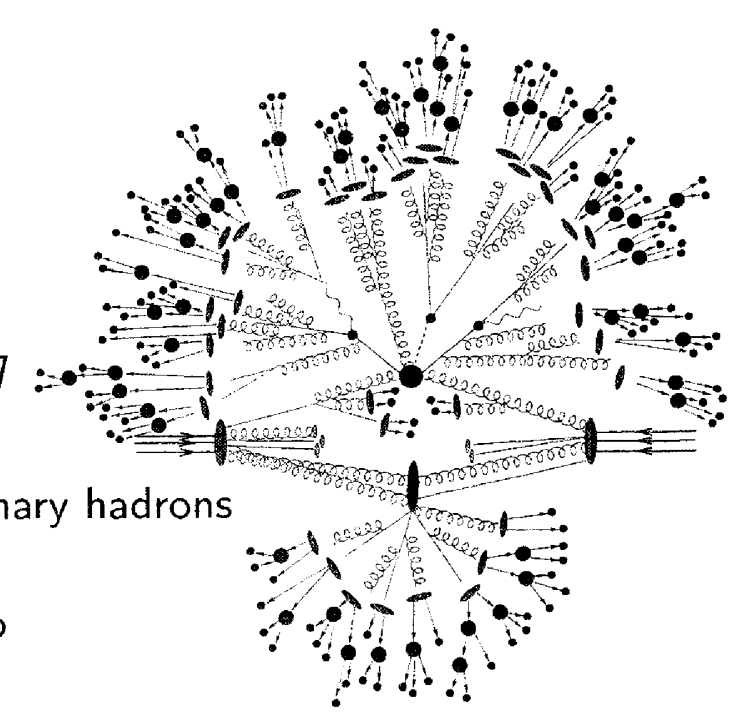

7 predictions at hadron level - comparable to experimental data if corrected for detector effects 


\section{Comparison with CDF data: $\mathrm{W}+$ jets production}

[T. AALTONEN ET AL., PRD 77 (2008) 011108]

- Monte Carlos need to be validated and tuned against most recent Tevatron data.

- Sherpa vs1.1.3 predictions normalized to total inclusive cross section. Two choices of PDFs.

- Tree-level ME+PS can reproduce $W+>=n$ jet xsecs to $20 \%$ after applying overall $K$ factor.
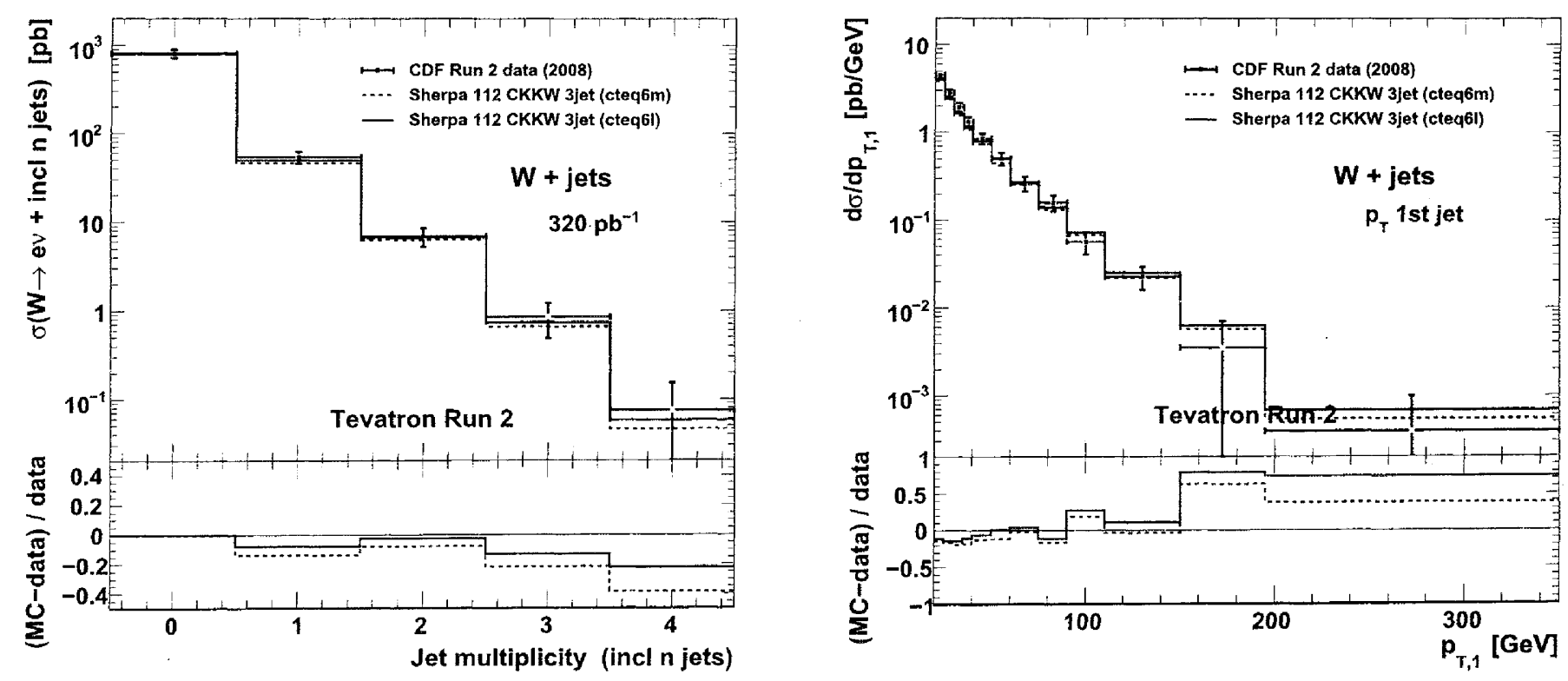


\section{Comparison with CDF data: $\mathbf{Z}+$ jets production}

$$
\text { ME\&TS :: COMIX + CSS }
$$

[T. AALTONEN ET AL., PRL 100 (2008) 102001]

\& Sherpa vs1.1 [CKKW] (left) compared with Sherpa vs1.2 [ME \&TS] (right).

\& Examples of jet observables: new approach better describes the data.

\& Sherpa predictions multiplied by constant $K$ factor, normalized to first-jet bin xsec.
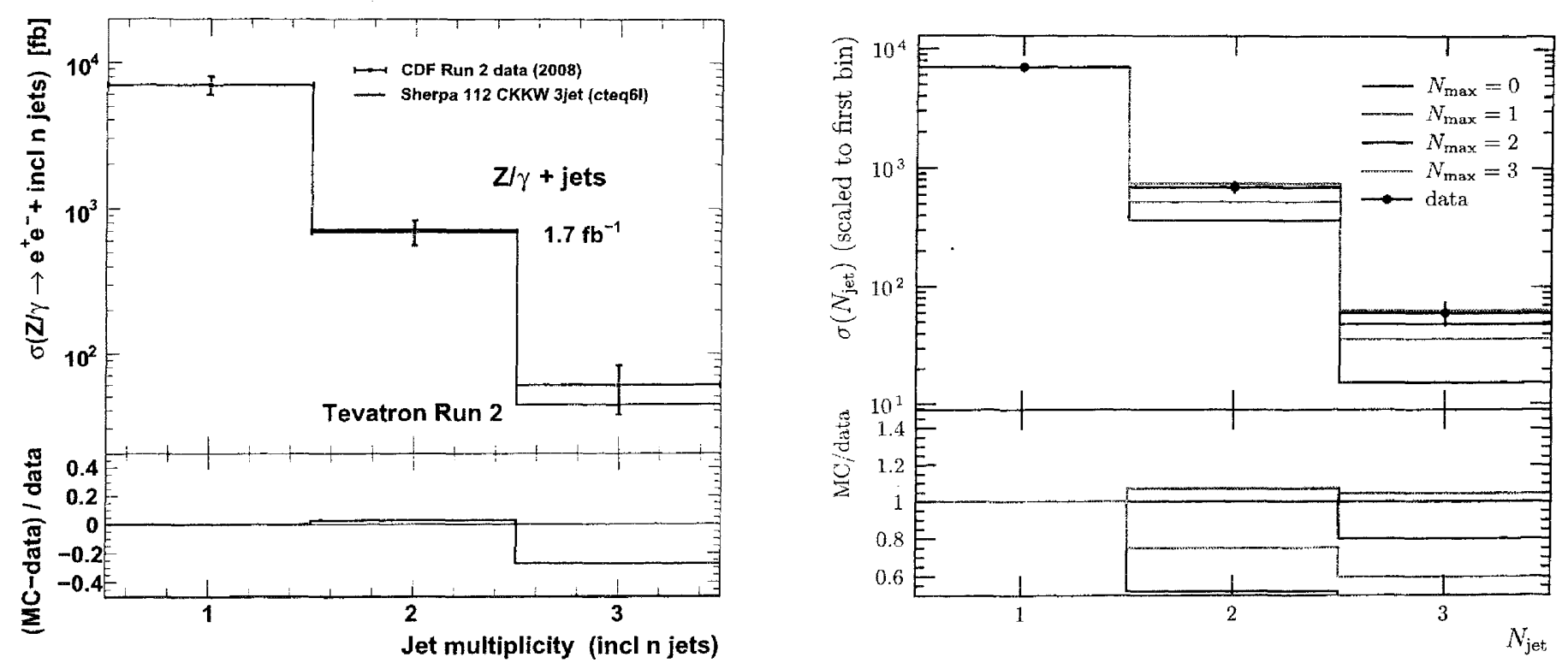


\title{
Z+jets production @ Tevatron Run2 energies
}

\author{
ME\&TS :: COMIX + CSS \\ [HÖCHE, KRAUSS, SCHUMANN, SIEGERT, JHEP 05 (2009) 053]
}

\& Merging systematics of total cross section (LO) has improved: $\quad \Delta \sigma_{\text {tot }} / \sigma_{\text {tot }}< \pm 3 \%$

1 Differential $k_{T}$ jet rates in $Q_{\text {cut }}=Q_{\text {jet }}$ variation @ hadron level. Note $N_{\max }=5$.

c $Q_{\text {cut }}$ variation now within $\pm 10 \%$. Note $\mu_{\mathrm{F}}^{2}=M_{e e}^{2}$ and $66 \mathrm{GeV}<M_{e e}<116 \mathrm{GeV}$.
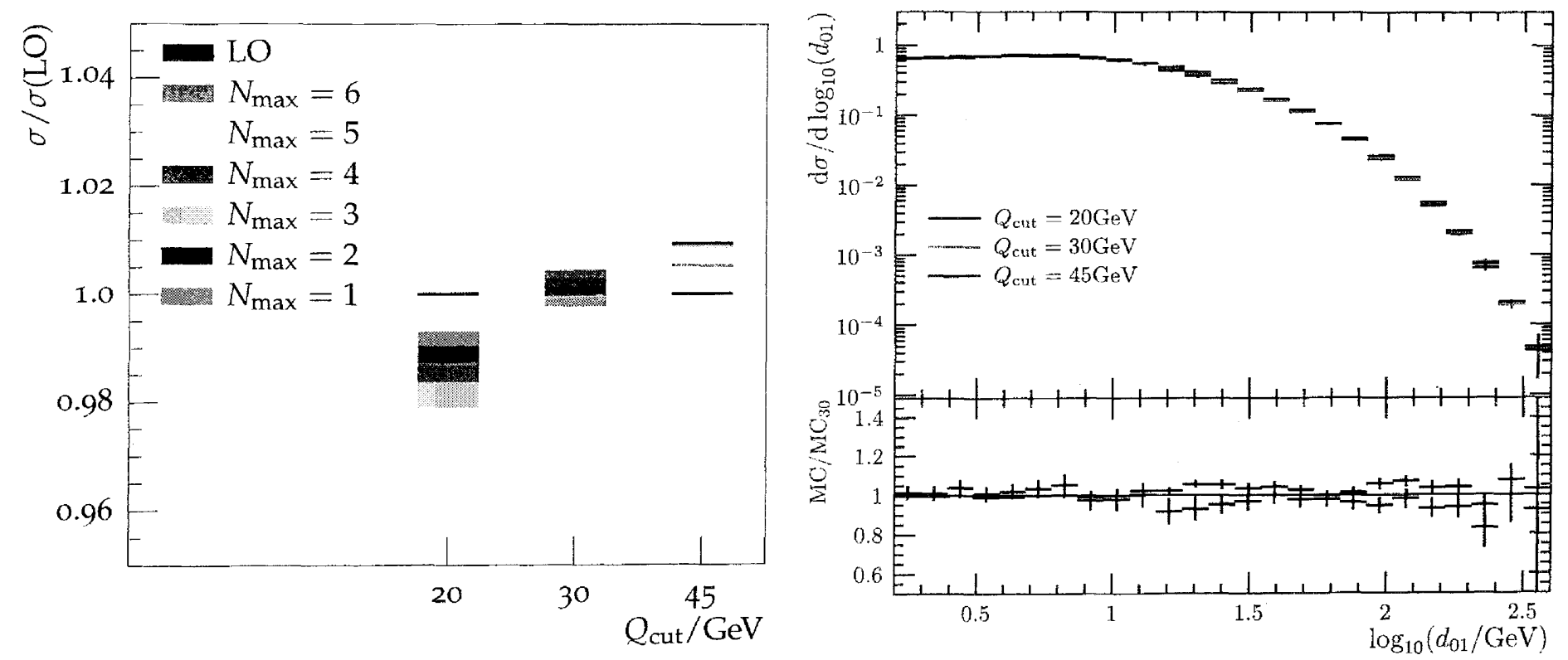


\section{Recent comparison of LHC predictions for W+3jets}

[HÖCHE, HUSTON, MAITRE, WINTER, ZANDERIGHI; LH09 PROCEED.: ARXIV:1003.1241]

- between BlackHat [Berger et al.], Rocket [Ellis, Melnikov, Zanderighi] and Sherpa [Gleisberg et al.]

- rather different scale choices at NLO yield > 20\% deviations ... impact on BSM searches!

- SHERPA's ME\&TS merging in good agreement with NLO once rescaled to NLO xsec
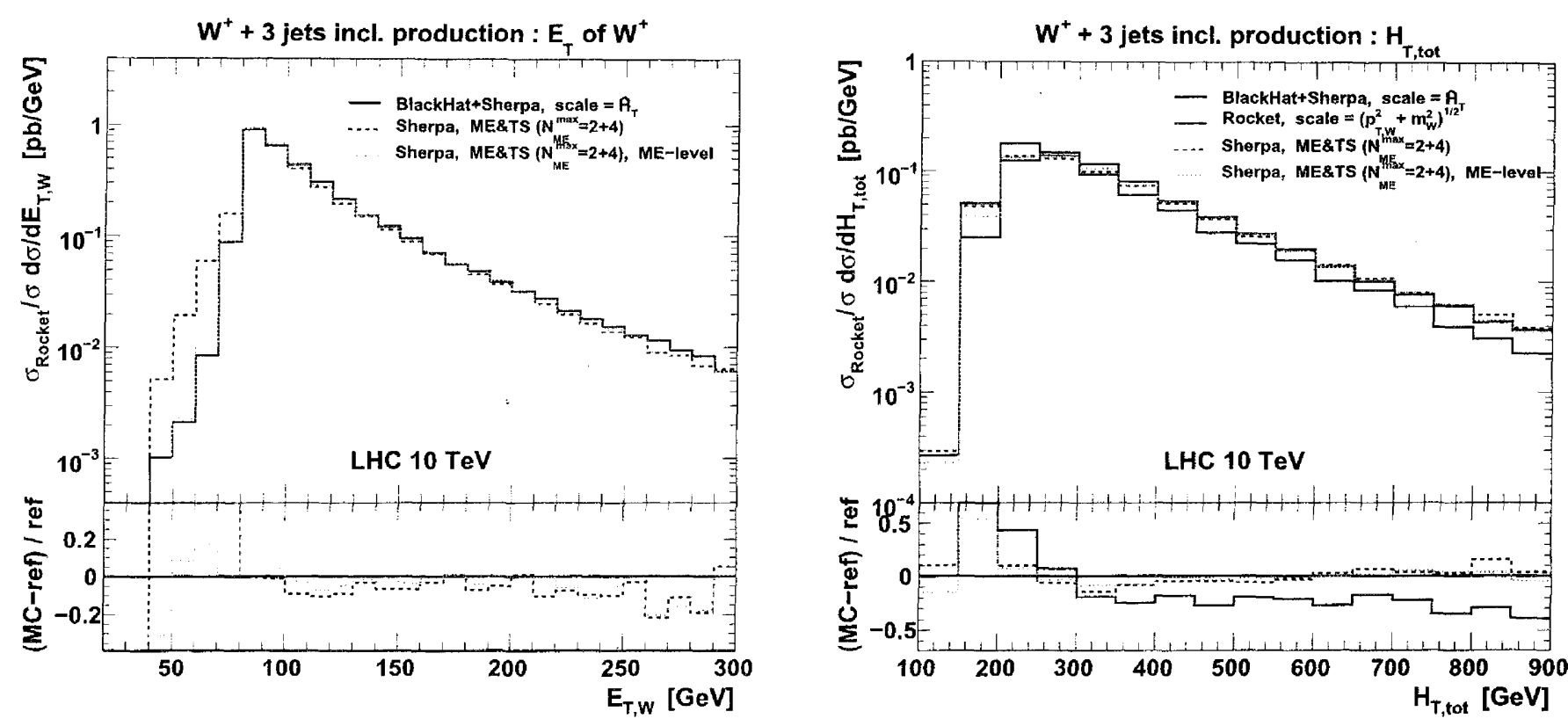


\title{
W Boson Physics
} and

Global Analysis of PDFs

\author{
C.-P. Yuan \\ Michigan State University
}

June24,2010@BNL

Workshop on

The Physics of $\mathrm{W}$ and $\mathrm{Z}$ Bosons 


\title{
Precision Electroweak Physics at Hadron Colliders
}

\author{
Physics of \\ Drell-Yan, $W$ and $Z$ Bosons
}




\section{$W$-boson physics}

(1) W-boson production and decay at hadron collider

(2) How to measure W-boson mass and width?

(3) High order radiative corrections:

QCD (NLO, NNLO, Resummation)

EW (QED-like, NLO)

(4) ResBos and ResBos-A 


\section{$\mathrm{W}$-boson production at hadron colliders}
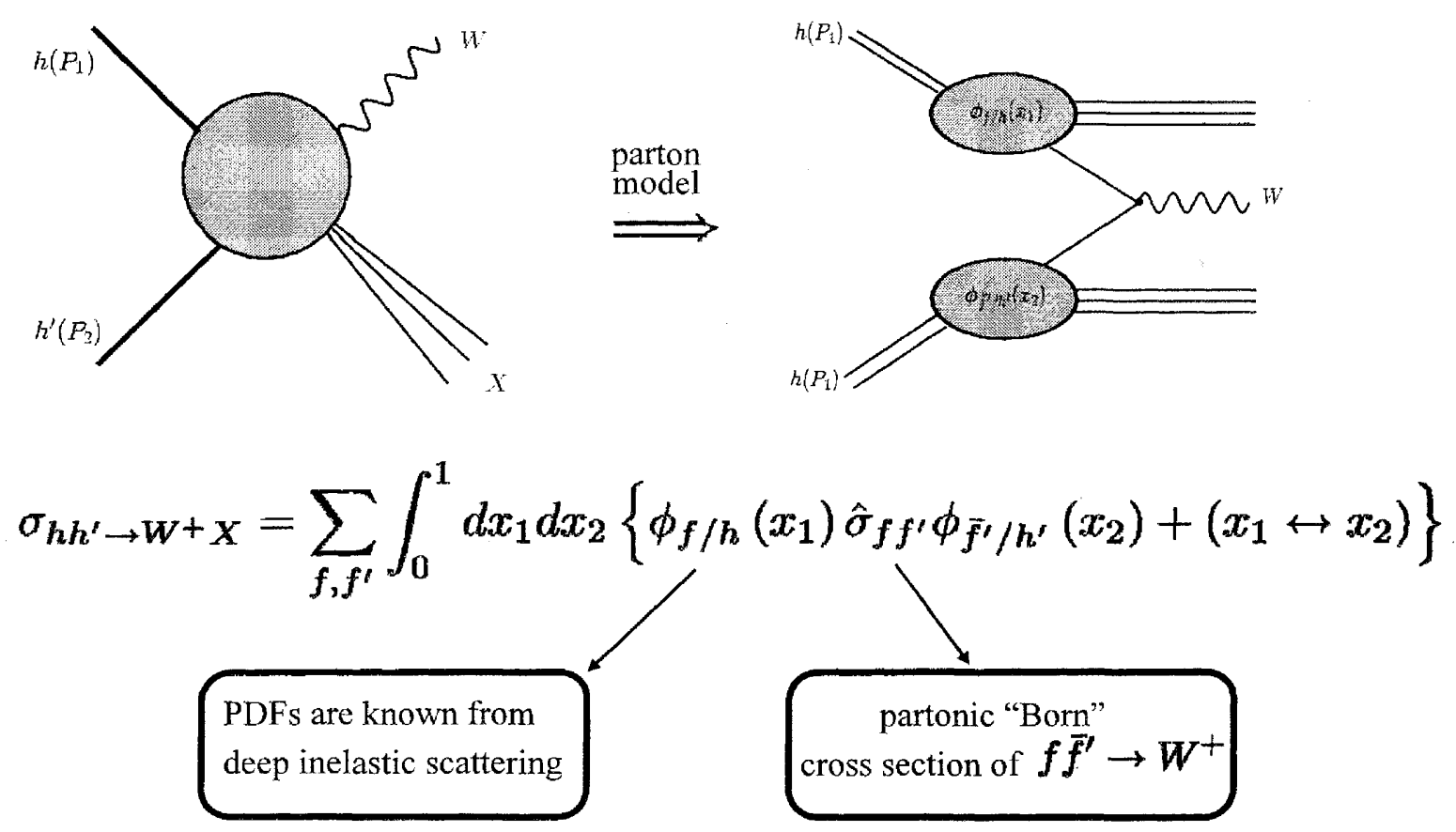


\section{$\mathrm{W}$-boson production at hadron colliders}

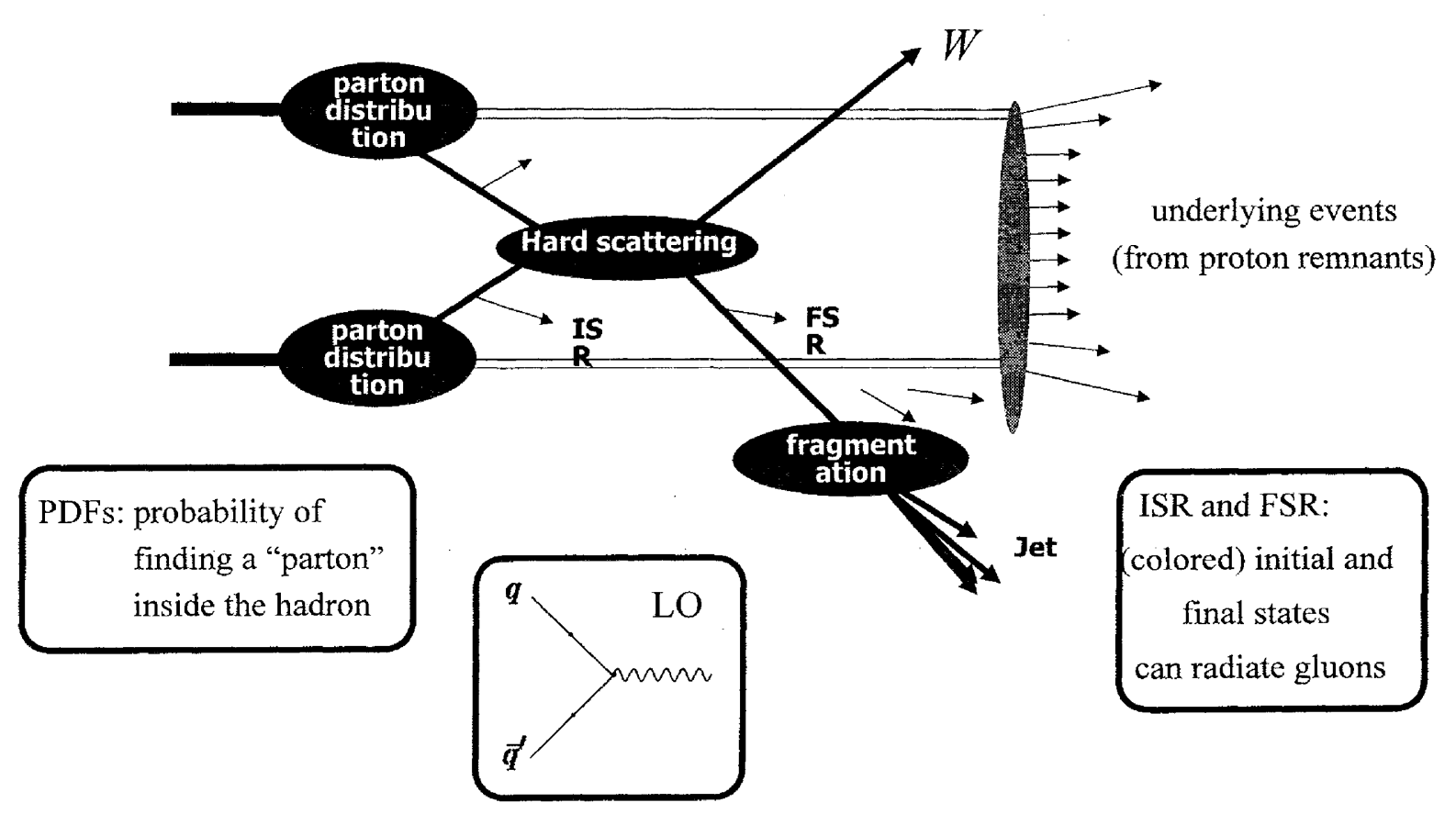




\section{Fixed order pQCD prediction}

$$
\begin{gathered}
\sigma=\frac{1}{2 S} \int \frac{d \xi_{A}}{\xi_{A}} \frac{d \xi_{B}}{\xi_{B}} f_{i / A}\left(\xi_{A}, \mu\right) f_{i / B}\left(\xi_{B}, \mu\right) \cdot d \hat{\sigma} \\
d \hat{\sigma}=\underbrace{|M|^{2}}(2 \pi)^{4} \delta^{(4)}(q-k-l) \frac{d^{3} q}{(2 \pi)^{3} 2 q_{0}} \\
\frac{\mathrm{d} \sigma}{\mathrm{d} q_{T}^{2} \mathrm{~d} y \mathrm{~d} Q^{2}}=\frac{1}{S} \int \frac{\mathrm{d} \xi_{A}}{\xi_{A}} \frac{\mathrm{d} \xi_{B}}{\xi_{B}} f_{i / A}\left(\xi_{A}, \mu\right) f_{i / B}\left(\xi_{B}, \mu\right) \\
\cdot\left(\frac{\pi^{2}}{Q^{2}}\right) \cdot \frac{|M|^{2}}{\mid} \cdot \delta\left(1-\frac{x_{A}}{\xi_{A}}\right) \cdot \delta\left(1-\frac{x_{B}}{\xi_{B}}\right) \\
\cdot \delta\left(q_{T}^{2}\right) \cdot \delta\left(Q^{2}-M_{W}^{2}\right) \\
Q \equiv \sqrt{Q^{2}}=\sqrt{q^{2}}, \mu=Q=M_{F}, x_{A}=\frac{Q}{\sqrt{S}} e^{y}, x_{B}=\frac{Q}{\sqrt{S}} e^{-y}
\end{gathered}
$$$$
s=\left(p_{A}+p_{B}\right)^{2}
$$$$
k=\xi_{A} p_{A}
$$$$
l=\xi_{B} p_{B}
$$ 


\title{
Prospects and Future of the STAR W Program
}

\author{
Joe Seele (MIT) for the \\ StAR Collaboration
}

The STAR experiment is planning a number of upgrades and measurements that will constrain the polarized anti-quark distributions in a polarized proton. The Forward GEM Tracker will add charged particle tracking in the forward rapidity allowing for charge sign identification and background rejection in the forward rapidity region. 


\section{"Predictions" for $A_{L}$ 's}

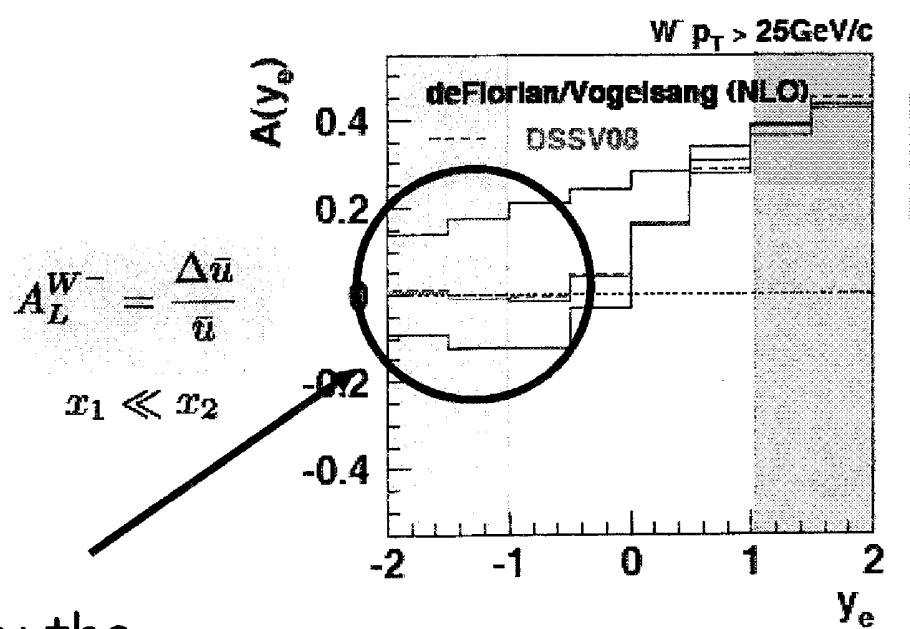

$$
A_{L}^{W^{-}}=-\frac{\Delta d}{d}
$$$$
x_{1} \gg x_{2}
$$

$$
\begin{gathered}
A_{L}^{W^{-}}=\frac{1}{2}\left(\frac{\Delta \bar{u}}{\bar{u}}-\frac{\Delta d}{d}\right) \\
x_{1}=x_{2}
\end{gathered}
$$

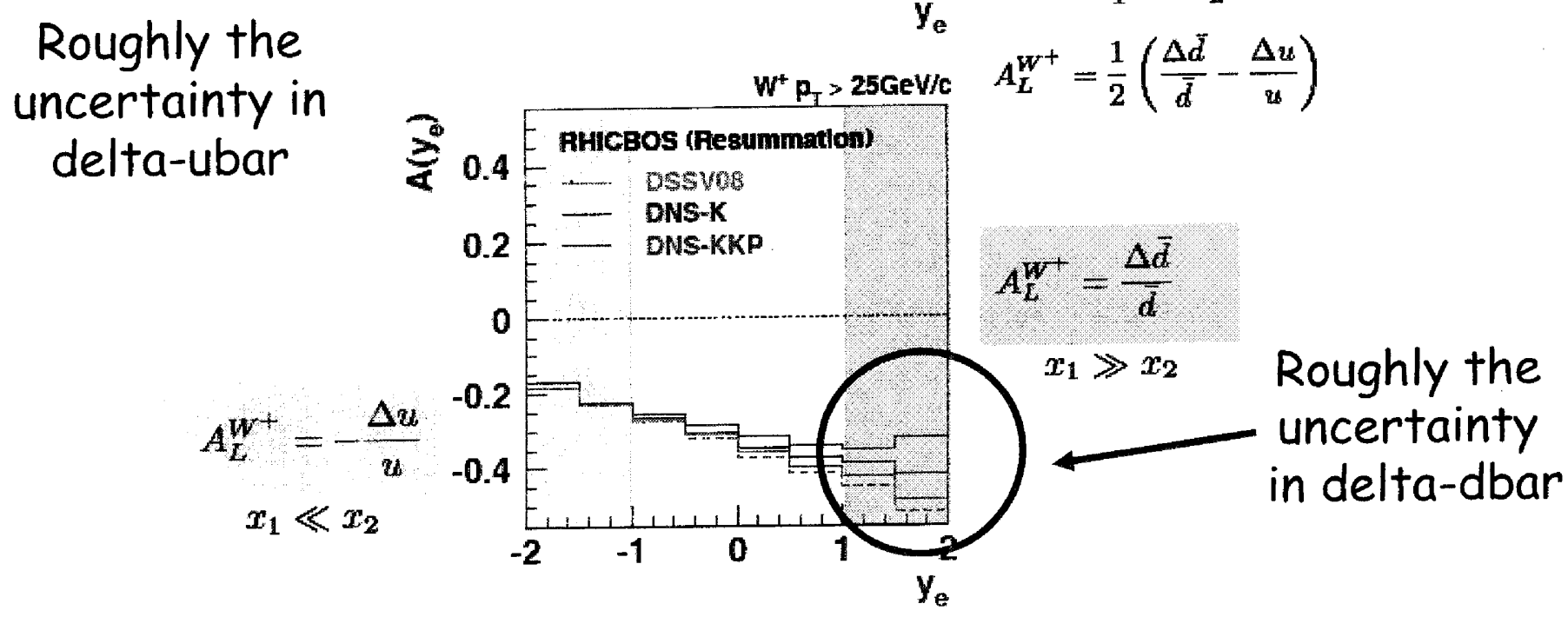




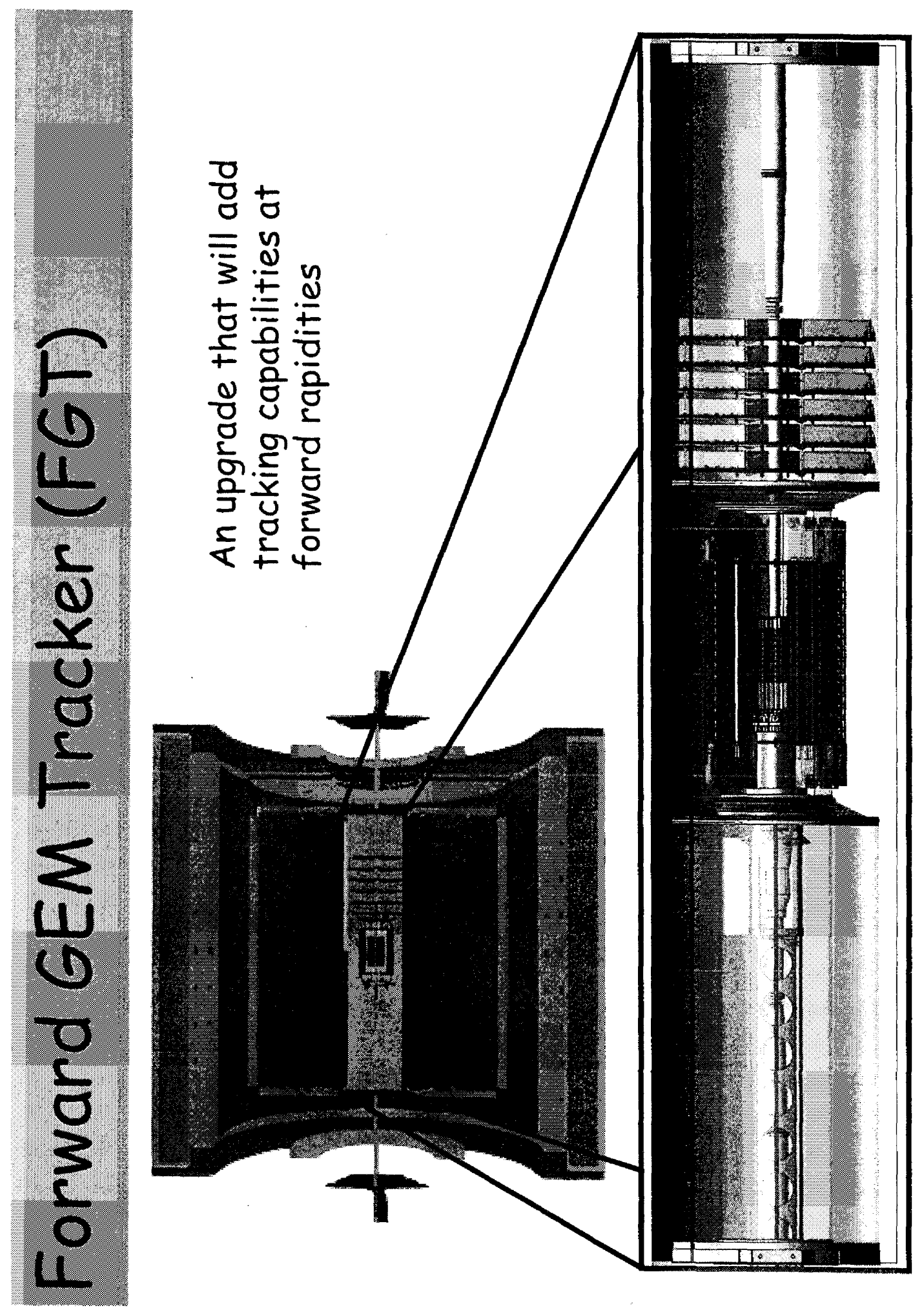




\section{GEM Foil Technology}

- High gain $\left(\sim 10^{6}\right)$

- Fast ( $(20$ ns FWHM)

- Low mass
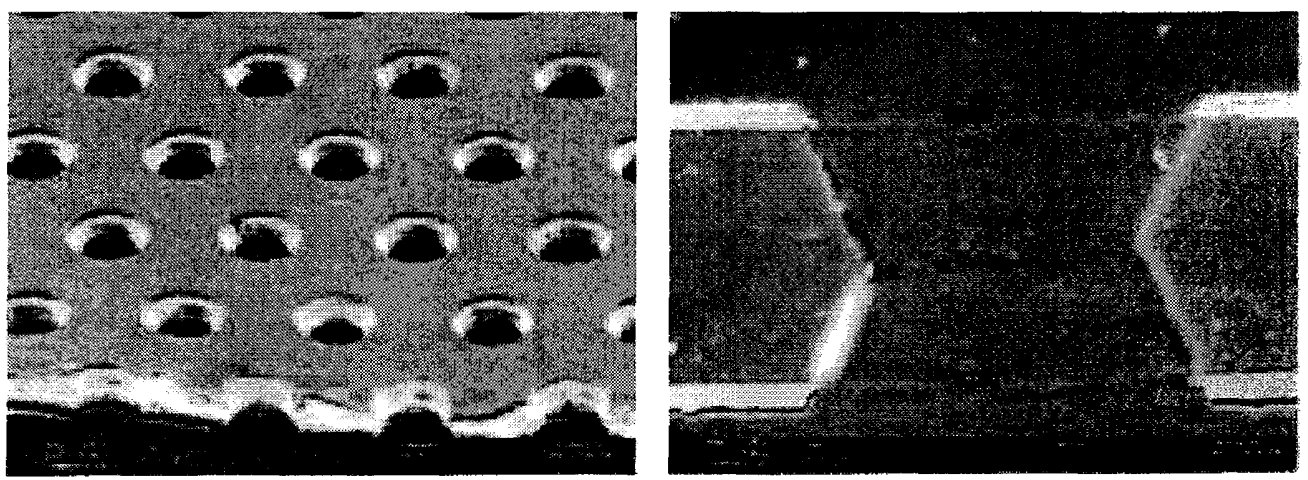

- Good spatial resolution

- Inexpensive

- Foils produced by CERN and Tech-etch

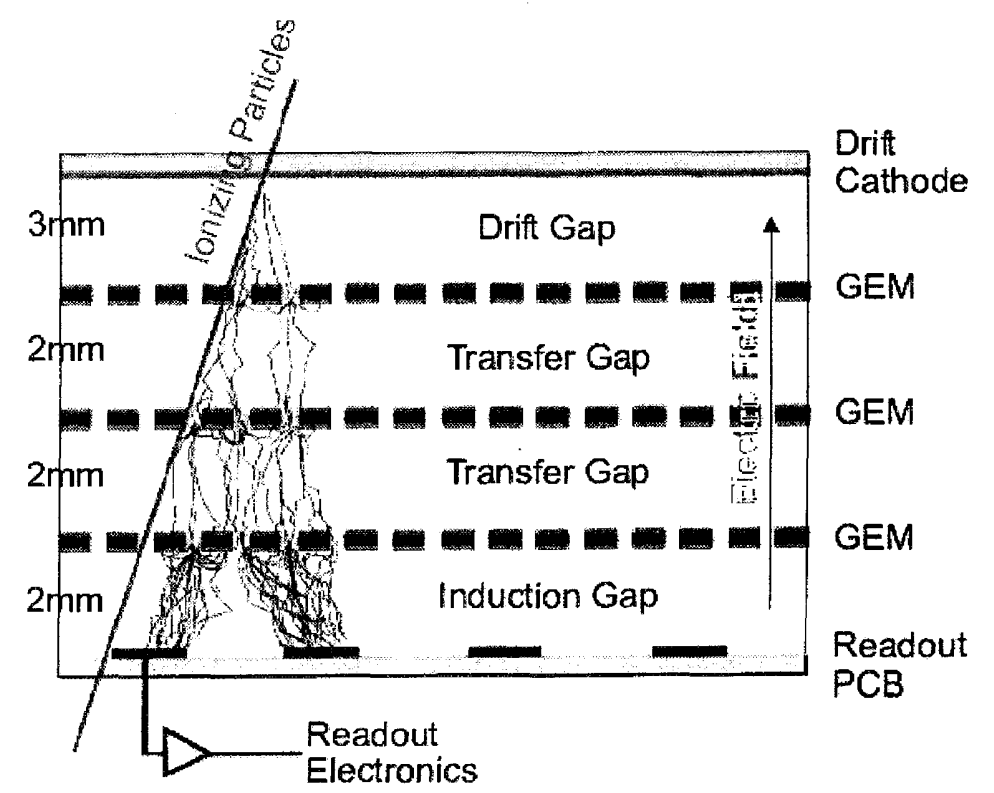




\section{Performance of FGT}

Charge sign reconstruction efficiency

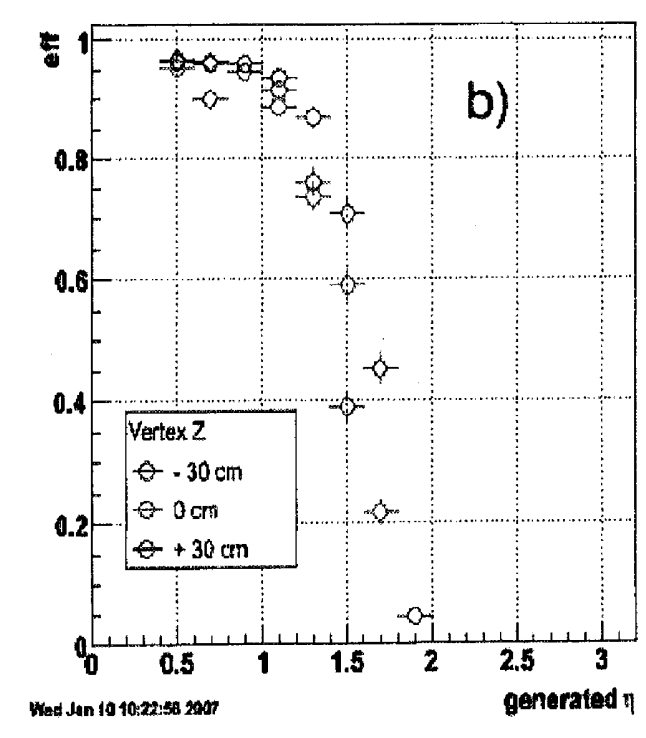

without FGT

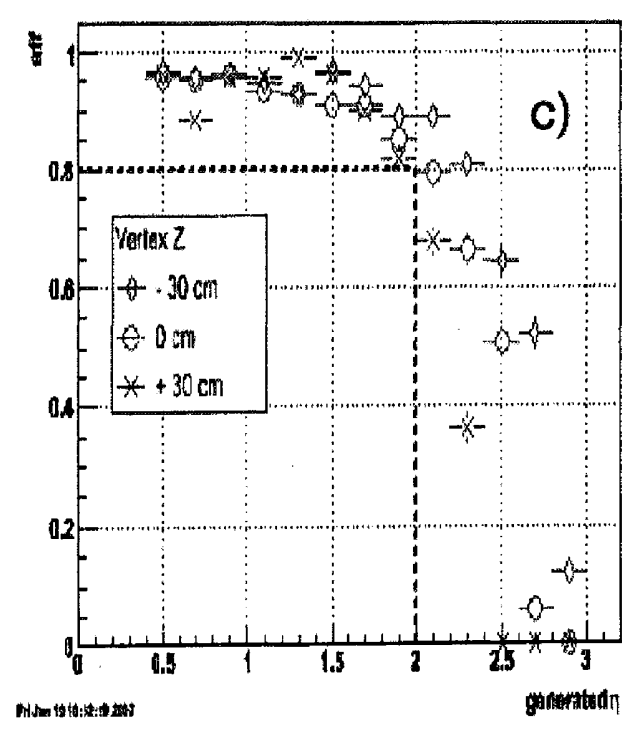

with FGT

The addition of the FGT will allow for charge sign identification at forward rapidities 


\section{Expected Data}

RHIC is planning to run 500 GeV polarized $p+p$ collisions in a multi-year effort to constrain the polarization of the anti-quarks in the proton

Calculations assume the demonstrated S/B (6 and 11) for the mid-rapidity projections and S/B 1 for the forward and backward rapidity projections

leption $|\pi|<1: 2$ beams, eff=0.65 wi gMHz RF, Run9 QCD bekg, thicbos o' $W^{*}, W^{\prime}=82,19$ ph

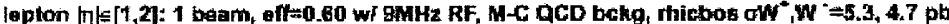

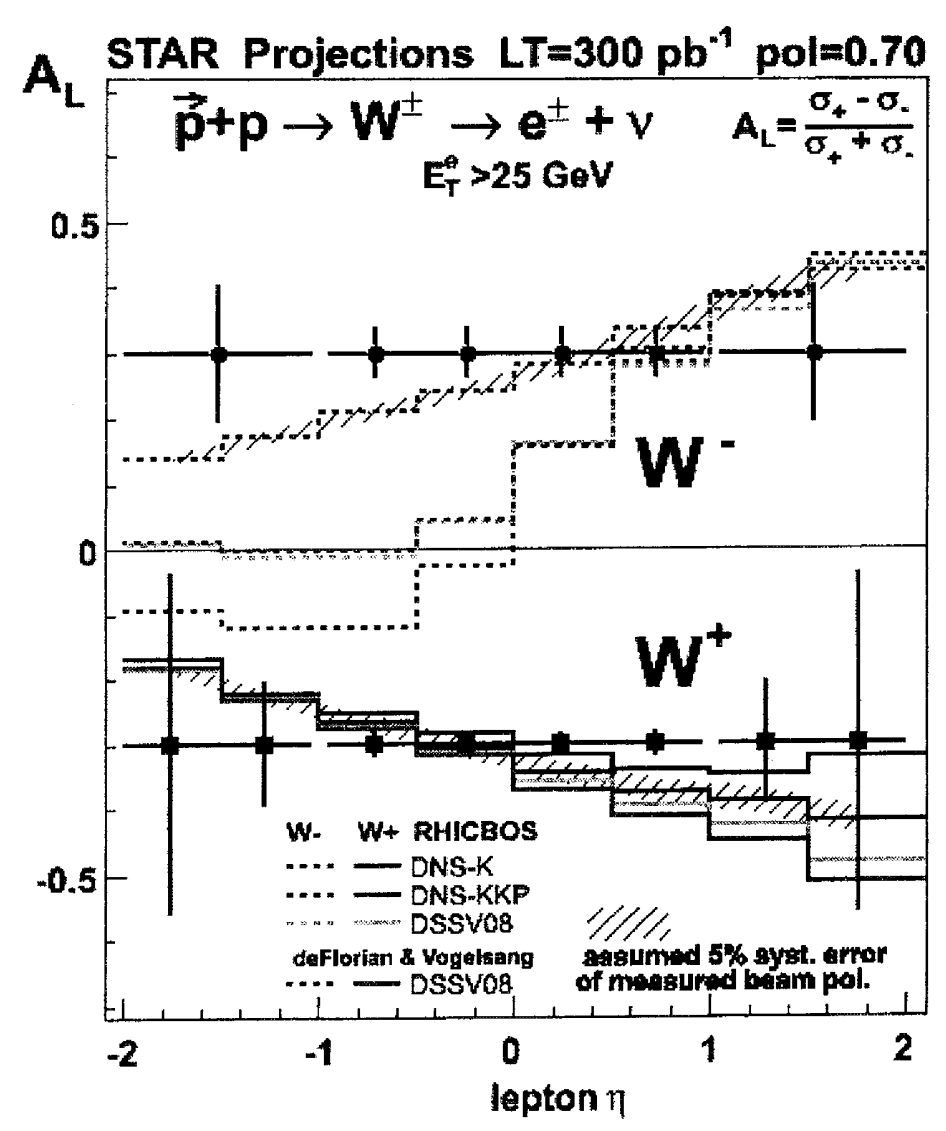




\title{
Prospects of the Forward W Measurement in Polarized pp Collisions at the RHIC-PHENIX Experiment
}

\author{
RIKEN Nishina Center for Accelerator-Based Science, \\ Wako, Saitama 351-0198, Japan \\ Fukao, Yoshinori
}

RHIC-PHENIX experiment aim to measure $A L$ in $W \rightarrow m$ process and impose significant constraint on polarized anti-quark PDF with $500 \mathrm{GeV}$ polarized pp collisions. One of the major upgrade work is the development of new W trigger system, which consists of MuTRG and RPC. We completed MUTRG and RPC3 North installation and will finish RPC3 South installation during 2010 shutdown period. The commissioning of the $W$ trigger system was performed with beam and cosmic ray, and final performance evaluation of full-chain trigger system is ongoing. In addition to the trigger development, new hadron absorber, which provide powerful background rejection, is in manufacture and will be installed in 2010 . Offline analysis and simulation for $W$ signal extraction are also in progress towards coming physics run in 2011 and future. 


\section{W Trigger System}

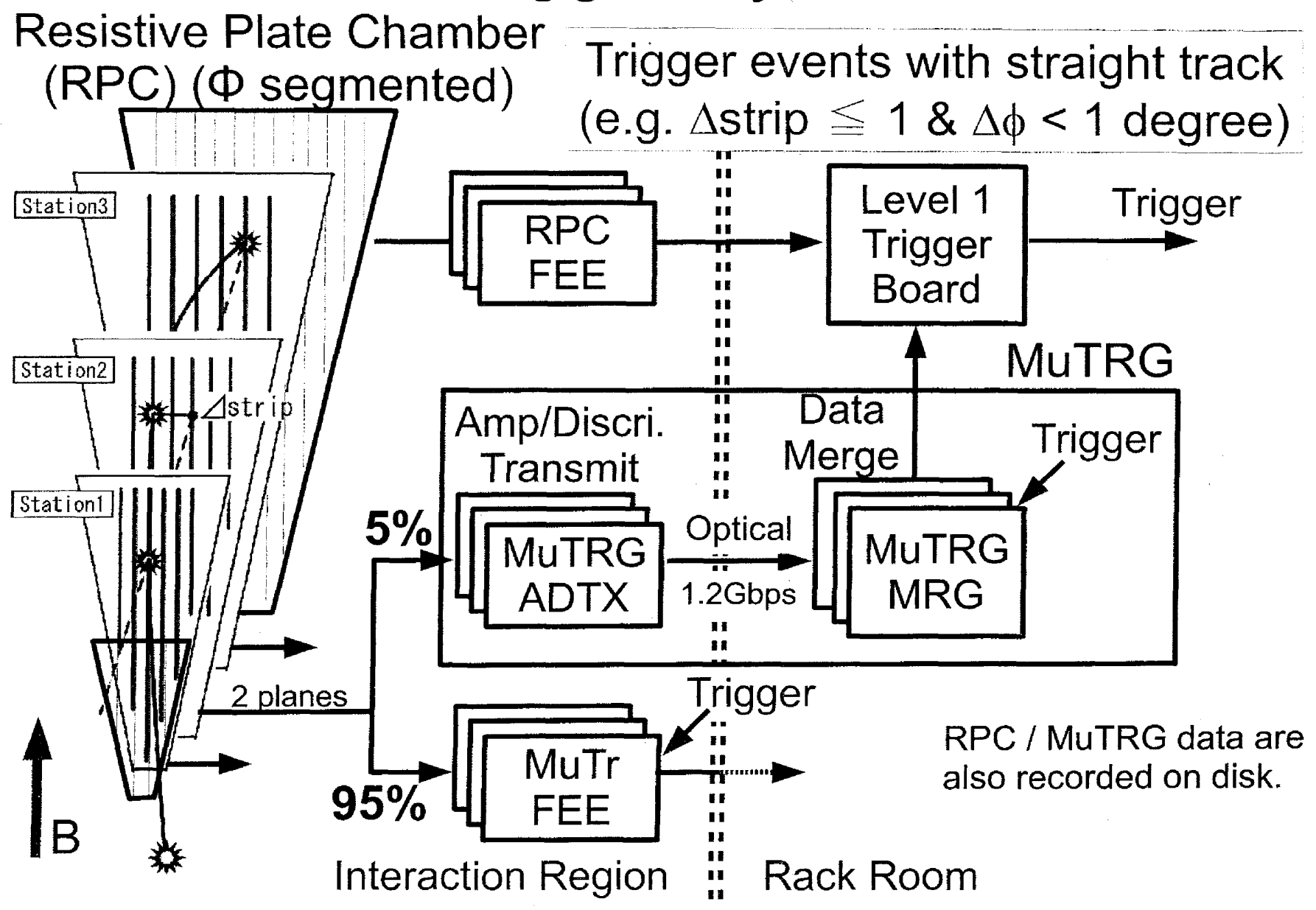




\section{Components of $\mathrm{W}$ trigger}

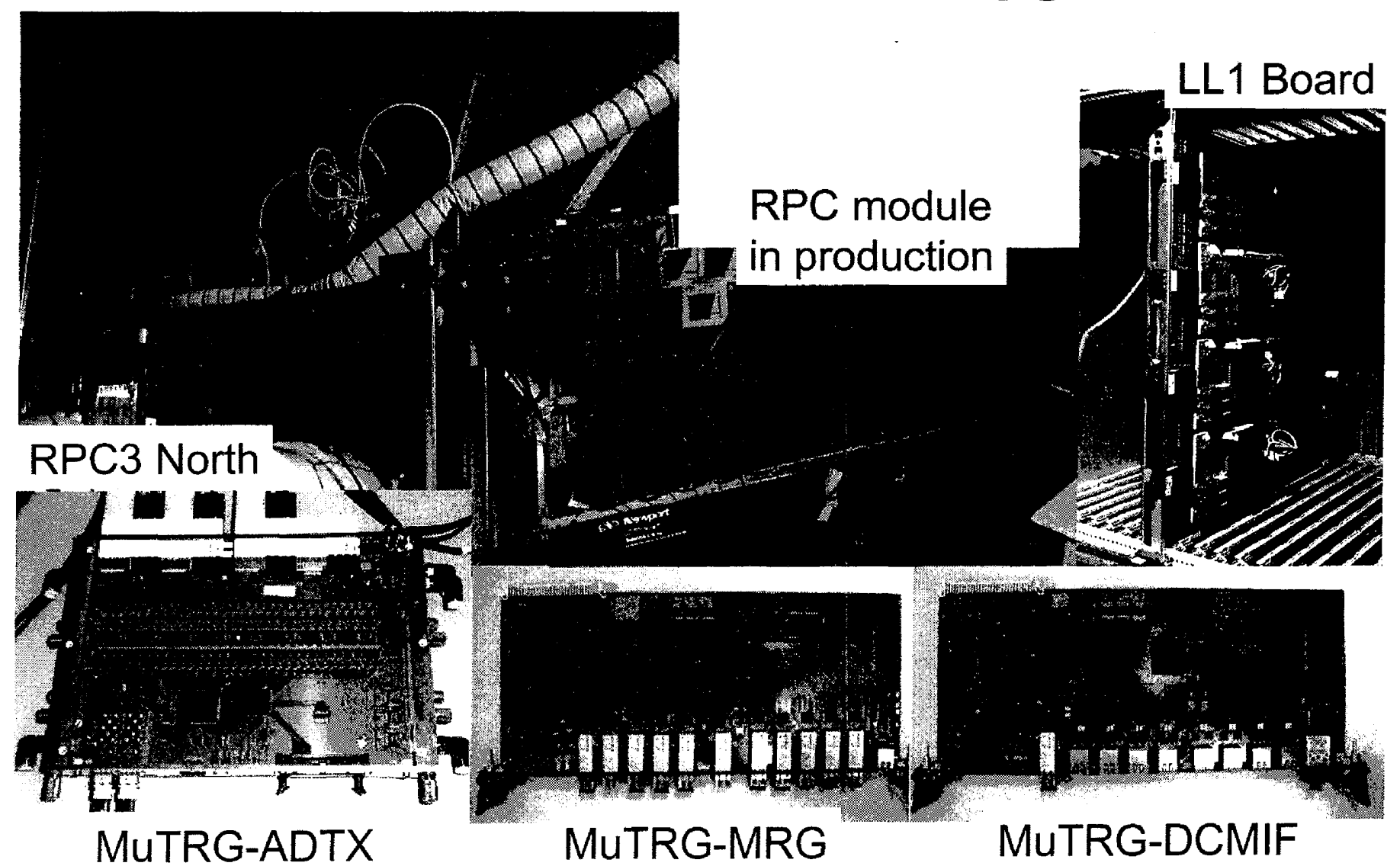




\section{Rejection Power with MuTRG only (MulD \& BBC \& MUTRG)}

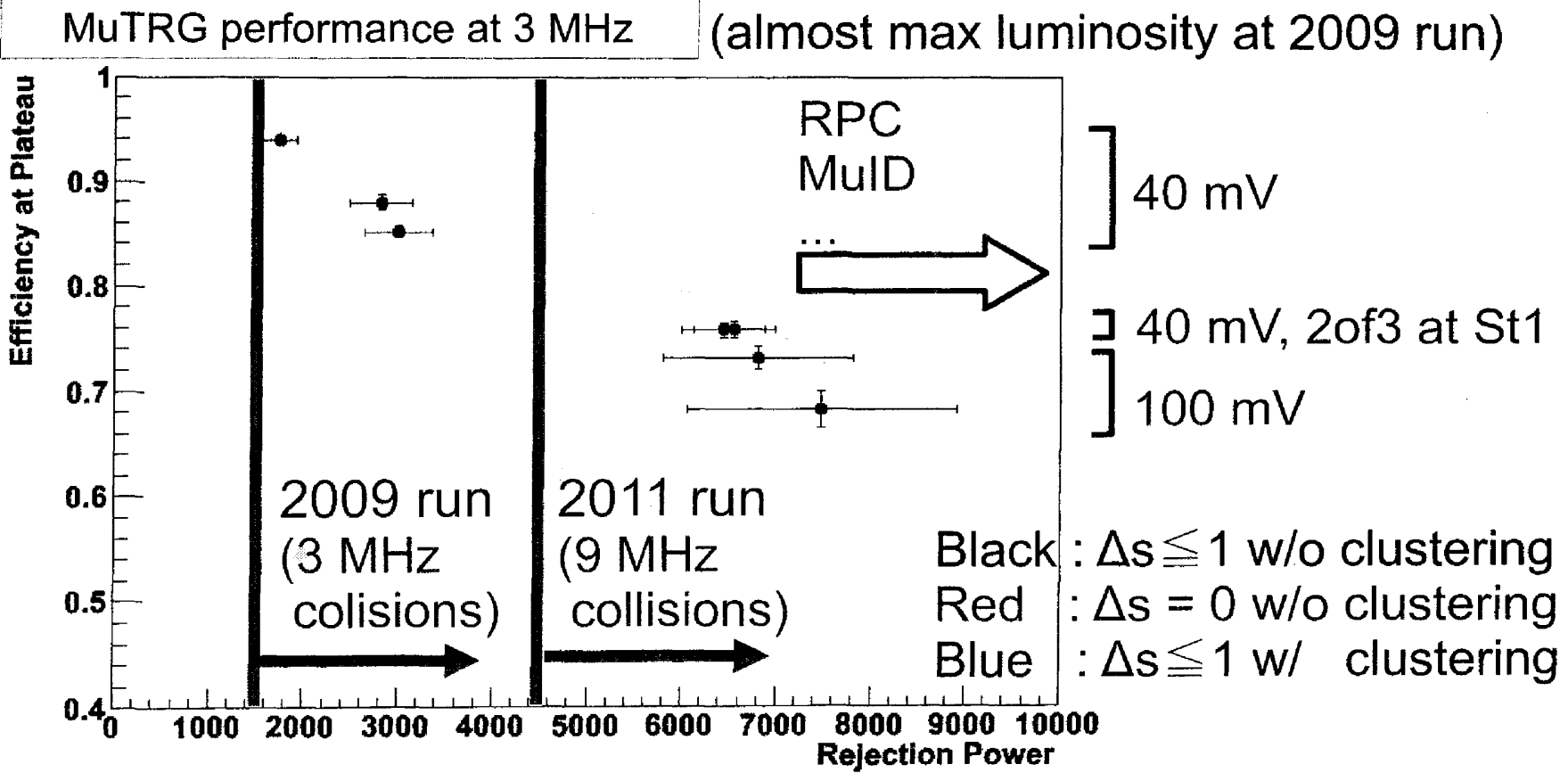

- Larger rejection power can be obtained by applying tight cut.

- More improvement is expected by RPC and other items. 


\section{RPC Module Performance}
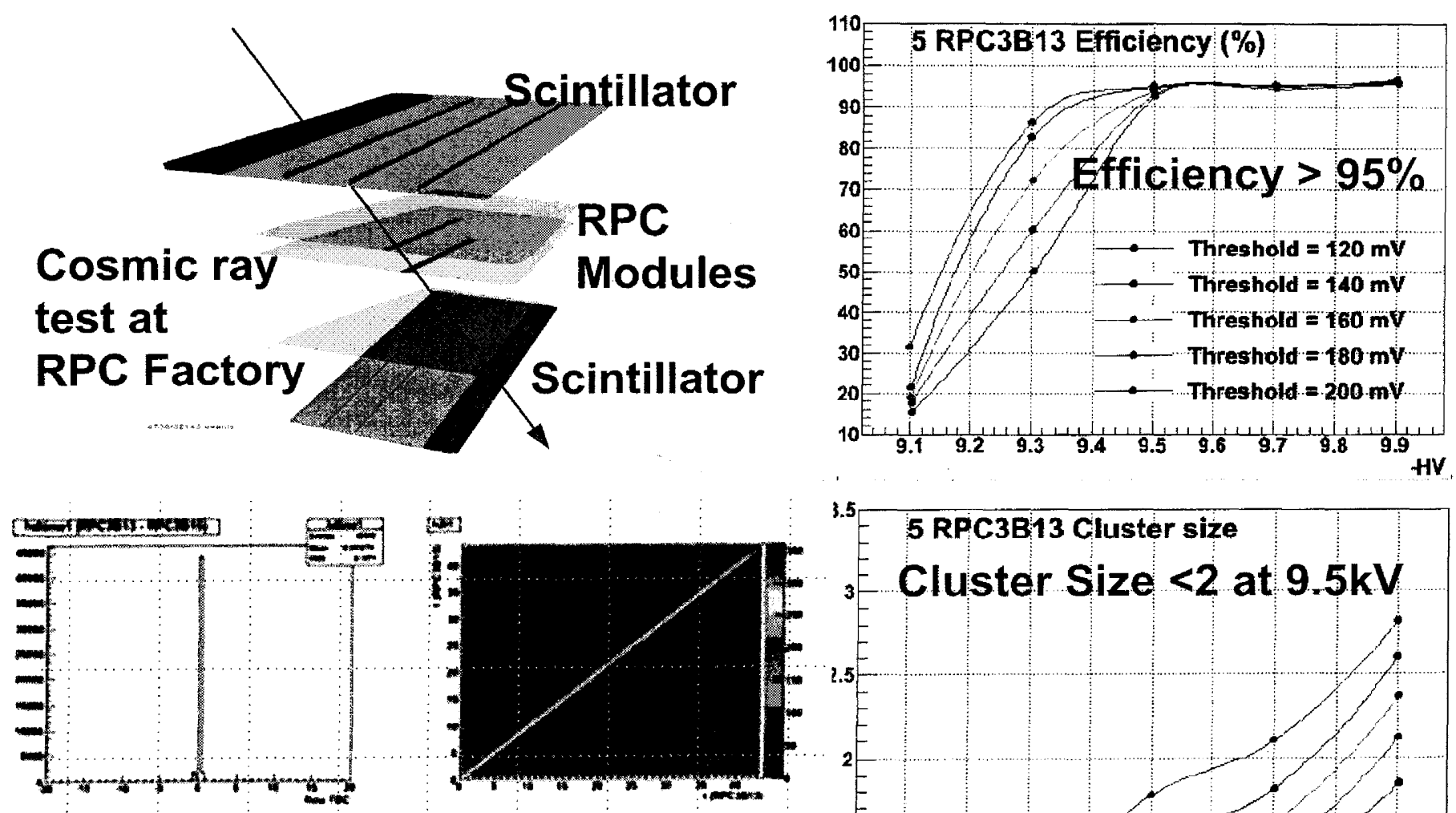

Timing difference between two RPC modules

Timing resolution $\sim 2 \mathrm{~ns}$

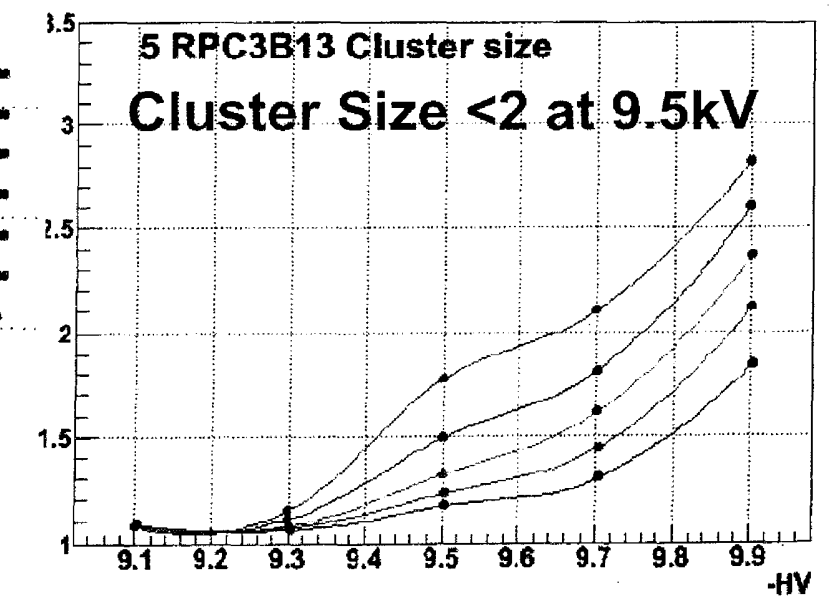




\section{Offline Analysis / Simulation}

Run $2011+2012$ Projection (150 pb-1, 50\% pol.)
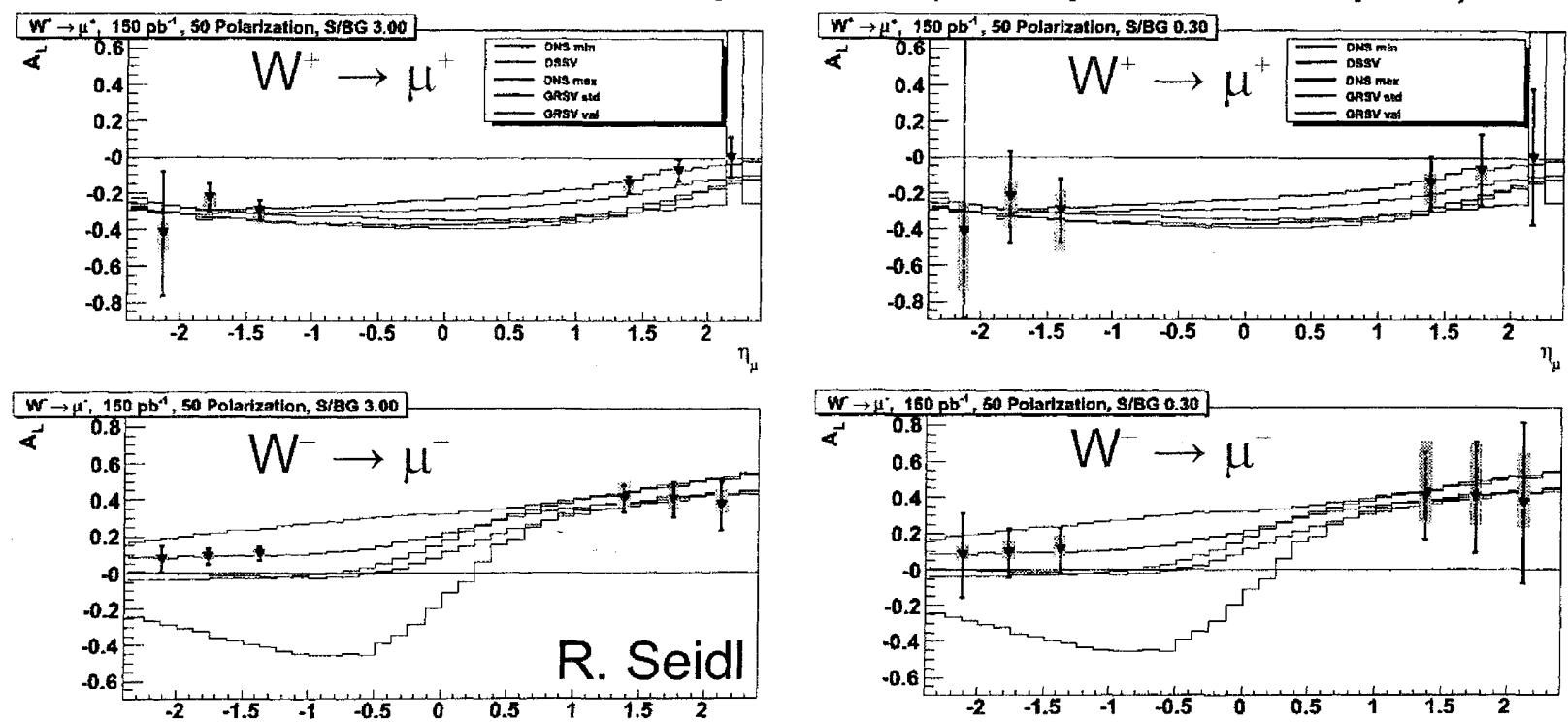

$\eta_{\mu}$ (muon pseudorapidity)

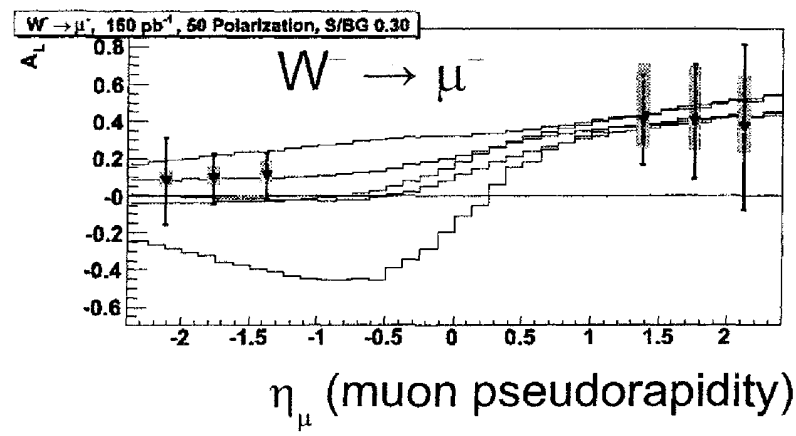

$\mathrm{S} / \mathrm{B}=3.0$ (optimistic)

$\mathrm{S} / \mathrm{B}=0.3$ (conservative)

$>$ Offline background evaluation using Run 2009 data and simulation study to optimize event selection is ongoing.

$>$ New Forward VTX detector will be installed and improve S/B. 


\title{
$A_{N}$ of $W$ Production in Polarized $p p$ Collisions
}

\author{
Zhong-Bo Kang \\ RIKEN BNL Research Center, Brookhaven National Laboratory, Upton, NY 11973, USA
}

Much of the predictive power of perturbative Quantum Chromodynamics (QCD) is contained in. factorization theorems. They normally include two assertions: a physical quantity can be factorized into perturbatively calculable short-distance hard parts convoluted with nonperturbative long-distance distribution functions; the universality of the nonperturbative functions. Predictions follow when processes with different hard scatterings but the same distribution functions are compared.

The phenomenon of single transverse-spin asymmetry (SSA), $A_{N} \equiv\left(\sigma\left(\vec{S}_{\perp}\right)-\sigma\left(-\vec{S}_{\perp}\right)\right) /\left(\sigma\left(\vec{S}_{\perp}\right)+\right.$ $\sigma\left(-\vec{S}_{\perp}\right)$ ), defined as the ratio of the difference and the sum of the cross sections when the spin vector $\vec{S}_{\perp}$ is flipped, was first observed in the hadronic $\Lambda^{0}$ production at Fermilab in 1976. Large SSAs, as large as $30 \%$, have been consistently observed in various experiments involving one polarized hadron at different collision energies.

One of the approach to describe the observed SSAs in QCD is so-called Transverse Momentum Dependent (TMD) factorization approach, which factorizes $\sigma\left(\vec{S}_{\perp}\right)$ in terms of the TMD parton distributions and attributes the SSAs to the nonvanishing Sivers function, the spin dependent part of TMD parton distribution. One of the most non-trivial feature is that the Sivers function could be process dependent (non-universal). It was predicted by Collins around 2002 on the basis of time-reversal and parity arguments that the quark Sivers function in semi-inclusive deep inelastic scattering (SIDIS) and in Drell-Yan process (DY) have the same functional form but an opposite sign, a time-reversal Inodified universality.

The experimental check of this time-reversal modified universality of the Sivers function would provide a critical test of the TMD factorization approach and our current understanding of the SSAs. Recently, the quark Sivers function has been extracted from data of SIDIS experiments by Anselmino et.al. Future measurements of the SSAs in DY production have been planned. In this talk, we present the SSAs of inclusive single lepton production from the decay of $W$ bosons, and show that the lepton SSAs is significant and measurable for a good range of lepton rapidity at RHIC. We find that the lepton SSAs are sharply peaked at transverse momentum $p_{T} \sim M_{W} / 2$ with $W$ mass $M_{W}$. This is because the most $W$ bosons at RHIC have $q_{T} \ll M_{W}$. On the other hand, leptons from heavy quarkonium decay and other potential backgrounds are unlikely to be peaked at the $p_{T} \sim M_{W} / 2$. Since the $W$ production and DY share the same Sivers function, we argue that the SSA of inclusive high $p_{T}$ leptons at $\mathrm{RHIC}$ is an excellent observable for testing the time-reversal modified universality. 
- Different gauge link for gauge-invariant TMD distribution in SIDIS and

$$
\begin{aligned}
& f_{q / h}\left(x \cdot \mathrm{k}_{\perp}, \vec{S}\right)=\int \frac{d y^{-} d^{2} y \perp}{(2 \pi)^{3}} e^{i x p^{+} y^{-}-i \mathrm{k}_{\perp} \cdot \mathrm{y}_{\perp}}\langle p, \vec{S}| \vec{w}\left(0^{-}, 0_{\perp}\right) \text { Gauge link } \frac{\gamma^{+}}{2} \psi\left(y^{-}, \mathbf{y}_{\perp}\right)|p, \vec{S}\rangle \\
& \text { - SIDIS: } \Phi_{n}^{\dagger}\left(\{+\infty, 0\}, \mathbf{0}_{\perp}\right) \Phi_{\mathbf{n}_{\perp}}^{\dagger}\left(+\infty,\left\{\mathbf{y}_{\perp}, \mathbf{0}_{\perp}\right\}\right) \Phi_{n}\left(\left\{+\infty, y^{-}\right\}, \mathbf{y}_{\perp}\right) \\
& \Phi_{n}^{\dagger}\left(\{-\infty, 0\}, \mathbf{0}_{\perp}\right) \Phi_{\mathbf{n}_{\perp}}^{\dagger}\left(-\infty,\left\{\mathbf{y}_{\perp}, \mathbf{0}_{\perp}\right\}\right) \Phi_{n}\left(\left\{-\infty, y^{-}\right\}, \mathbf{y}_{\perp}\right)
\end{aligned}
$$

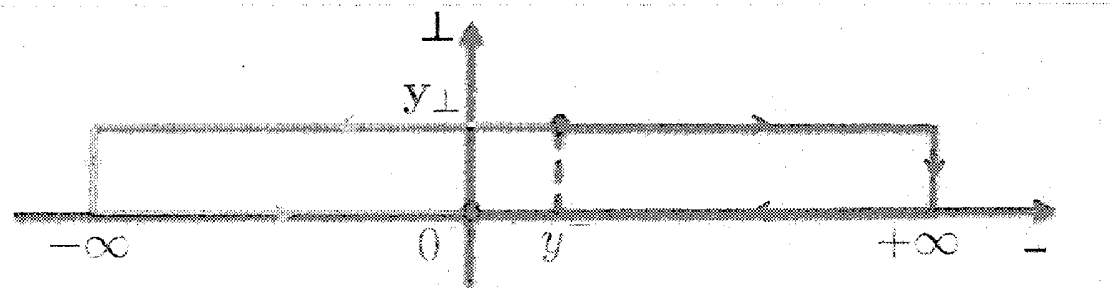

$$
\text { Wilson Loop } \sim \exp \left[-i g \int_{\Sigma} d \sigma^{\mu \nu} F_{\mu \nu}\right] \quad \text { Area is NOT zero }
$$
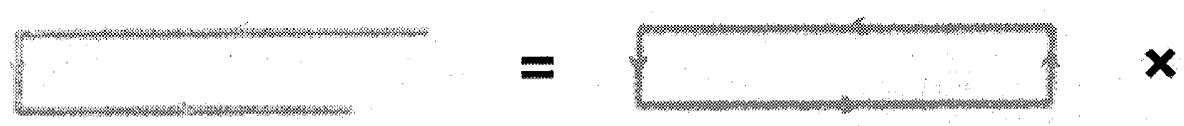

. For a fixed spin state:

$$
f_{q / h^{\uparrow}}^{\operatorname{SIDIS}}\left(x, \mathbf{k}_{\perp}, \vec{S}\right) \neq f_{q / h^{\uparrow}}\left(x, \mathbf{k}_{\perp}, \vec{S}\right)
$$


Time-reversal modified universality of the Sivers function

- Relation between Sivers functions in SIDIS and DY

* From P and T invariance:

$$
f_{q / h}^{\operatorname{SIDIS}}\left(x, \mathbf{k}_{\perp}, \vec{S}\right)=f_{q / h^{\circ}}^{\mathrm{DY}}\left(x, \mathbf{k}_{\perp},-\vec{S}\right)
$$

- Spin-averaged parton distribution function is universal

- From definition:

$$
f_{q / h^{\top}}\left(x, \mathbf{k}_{\perp}, \vec{S}\right) \equiv f_{q / h}\left(x, k_{\perp}\right)+\frac{1}{2} \Delta^{N} f_{q / h^{\top}}\left(x, k_{\perp}\right) \vec{S} \cdot \hat{p} \times \hat{\mathbf{k}}_{\perp}
$$

- One can derive:

$$
\Delta^{N} f_{q / h^{*}}^{\operatorname{SIDIS}}\left(x, k_{\perp}\right)=-\Delta^{N} f_{q / h}^{\mathrm{DY}}\left(x, k_{\perp}\right)
$$

Most critical test for TMD approach to SSA 
- Difference between initial and final state interactions

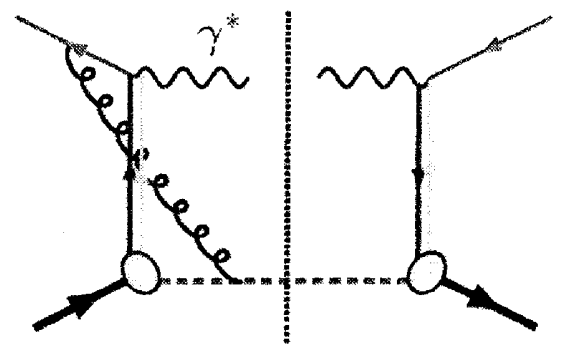

$$
p^{\uparrow}+p \rightarrow\left[\gamma^{*} \rightarrow \ell^{+} \ell^{--}\right]+X
$$

DY: repulsive

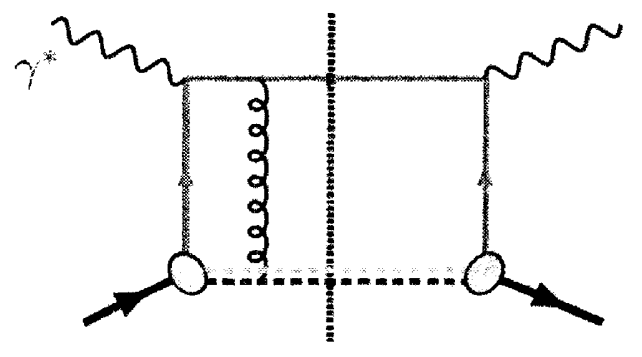

$\ell+p^{\uparrow} \rightarrow \ell+\pi+X$

SIDIS: attractive

$$
\Delta^{N} f_{q / h^{\top}}^{\operatorname{SIDIS}}\left(x, k_{\perp}\right)=-\Delta^{N} f_{q / h^{-}}^{\mathrm{DY}}\left(x, k_{\perp}\right)
$$

- Sign change:

- Test of TMD factorization

* Test of current understanding of SSA 


\section{SSA of lepton from $W$ decay: rapidity dependence}

m SSA of inclusive lepton is still sufficient for measurement:

Keno, Qu, PRL 103, 172001 (2009)
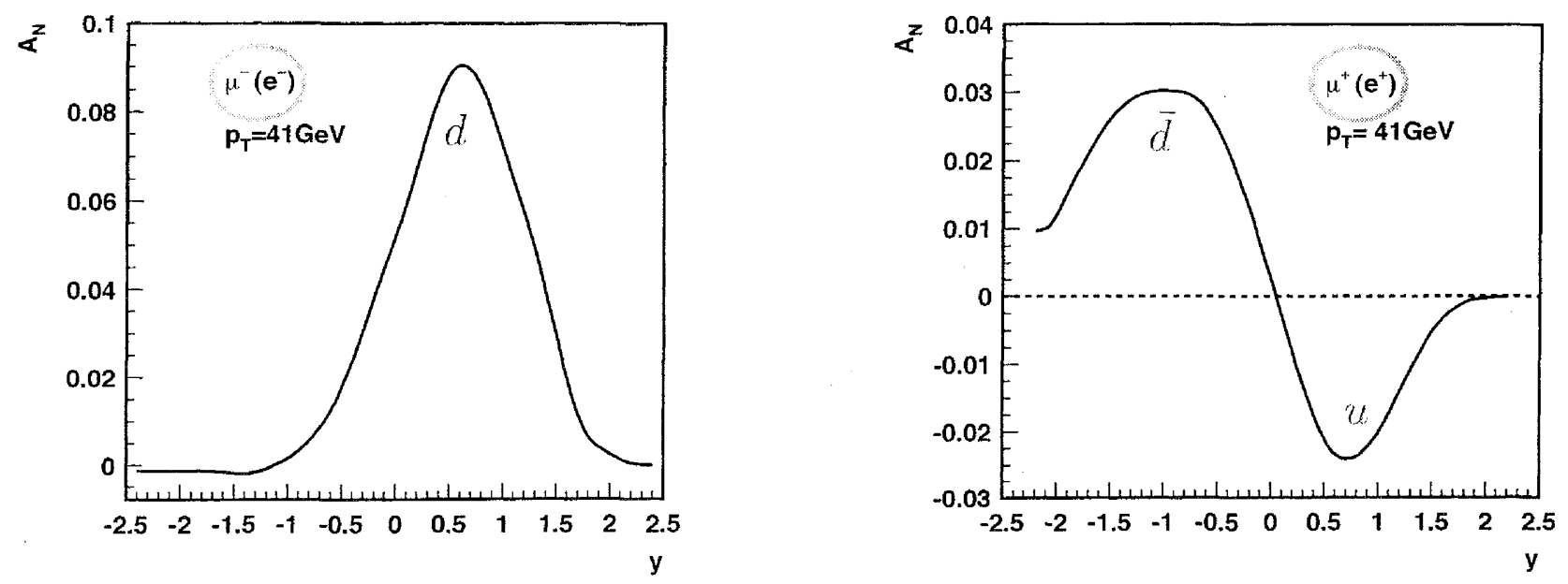

- Good flavor separation:

* $\mu^{-}\left(e^{-}\right)$at central-forward rapidity is sensitive to d Sivers function

- $\mu+\left(e^{+}\right)$at forward is sensitive to u Sivers function, at backward is sensitive to $d$-bar Sivers function 
SSA of lepton from $W$ decay: $p_{T}$ dependence

- pr behavior of SSA of leptons:
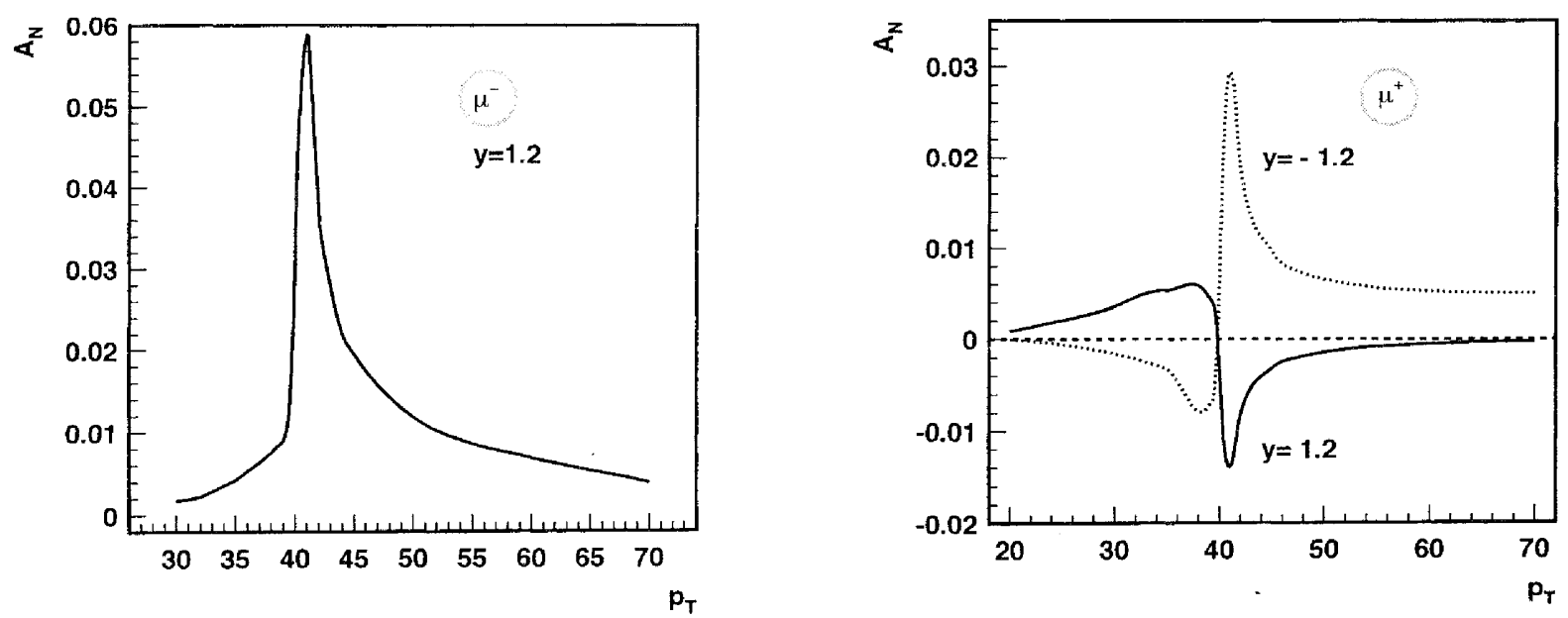

- inherit the key features of W asymmtry

" sharply peeked around pT M M $/ 2$, should help control the potential background 


\section{Precision Measurement of the $W$ Mass and New Physics}

1. Why?

2. $W$ Mass: Status and Measurement Techniques

3. Status of Theory Calculations for $W / Z$ Production

4. Conclusions

Ulrich Baur

State University of New York at Buffalo 


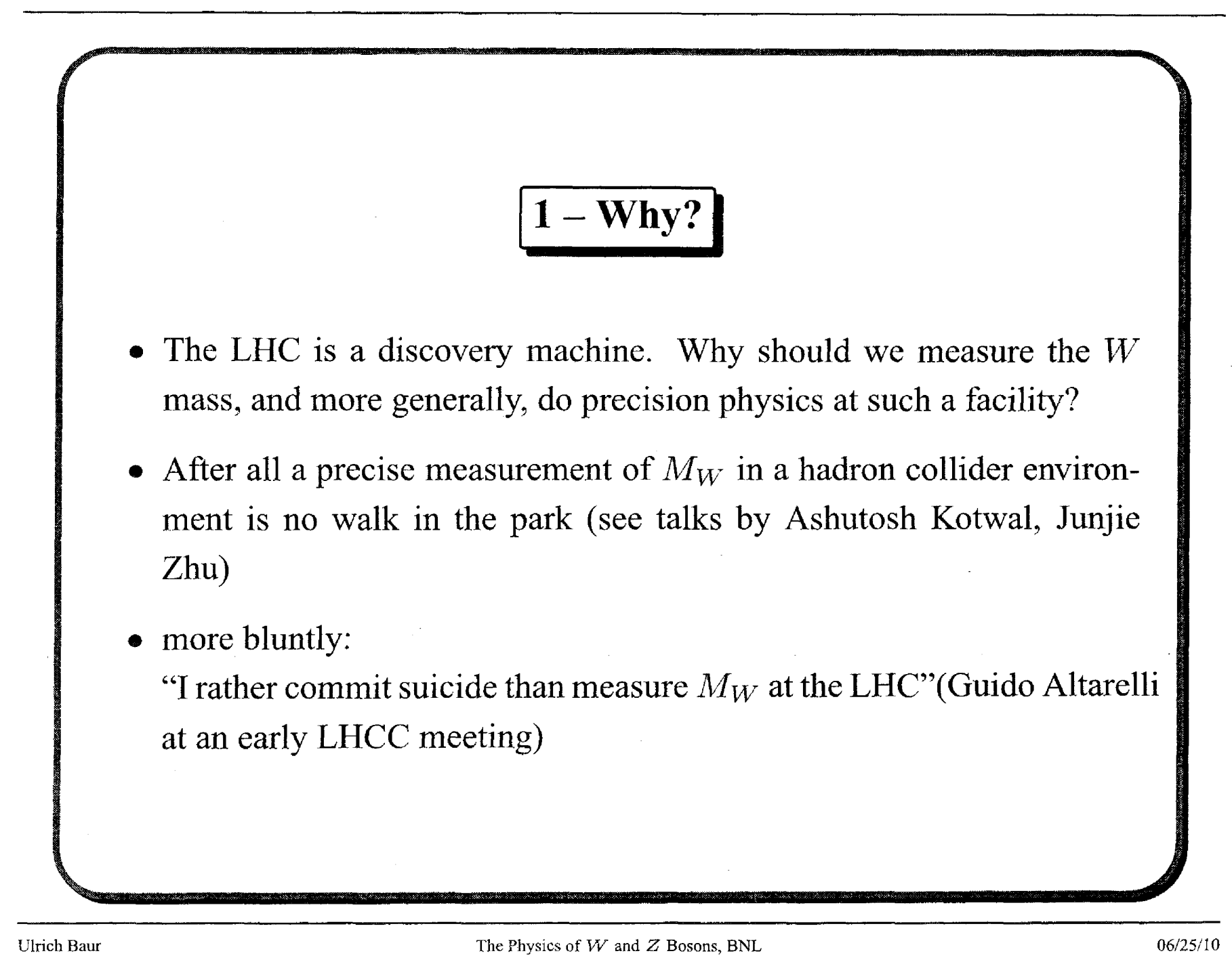


- Which measurements are of interest?

$-m_{t}, M_{W}$ and $\sin ^{2} \theta_{W}$

$\rightarrow$ make it possible to constrain the mass of the SM Higgs boson: winter 2010: $M_{H}<155 \mathrm{GeV}(95 \% \mathrm{CL})$ one-loop corrections to $M_{W}$ and $\sin ^{2} \theta_{W}$ depend logarithmically on $M_{H}$

$\rightarrow$ thus providing a consistency check on the SM (once a Higgs boson candidate has been observed)

$\rightarrow$ may give hints of new physics, or provide constraints on new physics models new particles contribute to the one-loop corrections 
Data in better agreement with SUSY models than SM

but this is not surprising as SUSY models have more free parameters

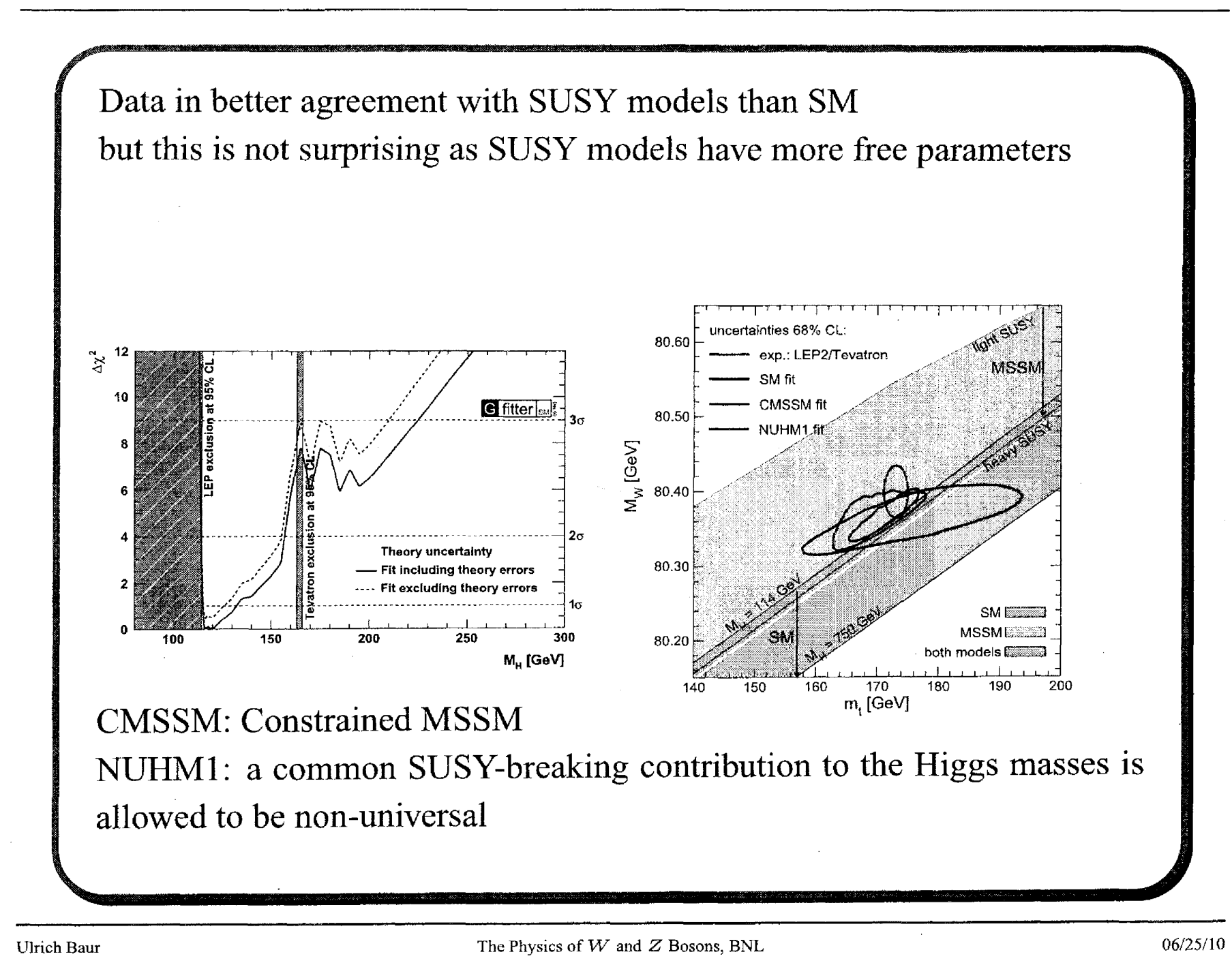


$W$ and $Z$ Production has been observed at the LHC $\mathrm{W} \rightarrow \mathrm{eV}$ candidate

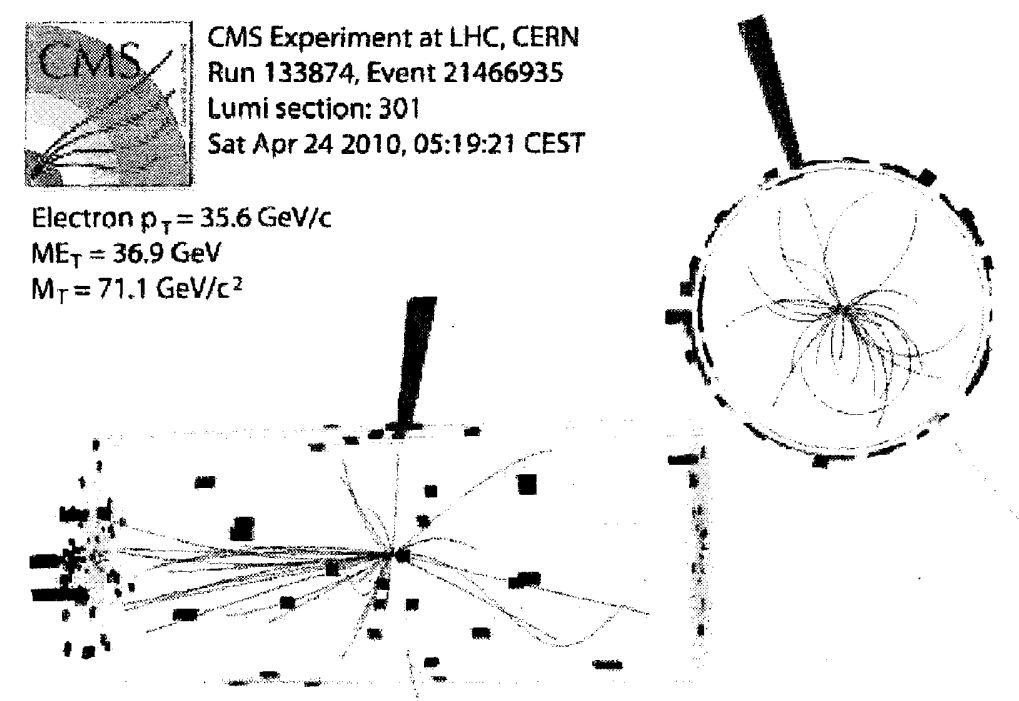




\section{4-Conclusions}

- $M_{W}$, together with $m_{t o p}$, make it possible to constrain the Higgs boson mass

- need $\delta M_{W}=\mathcal{O}(10 \mathrm{MeV})$ to match anticipated precision for $m_{t o p}$

- sensitive to new physics via loop corrections

- measuring $M_{W}$ at the LHC is non-trivial and may require special runs (deuterium, helium) and/or special detector configurations (reverse magentic field)

- EW radiative corrections affect the $M_{T}$ line shape and thus the $W$ mass extracted from data

- need better understanding how to combine calculations of QCD and EW corrections into one unified generator 


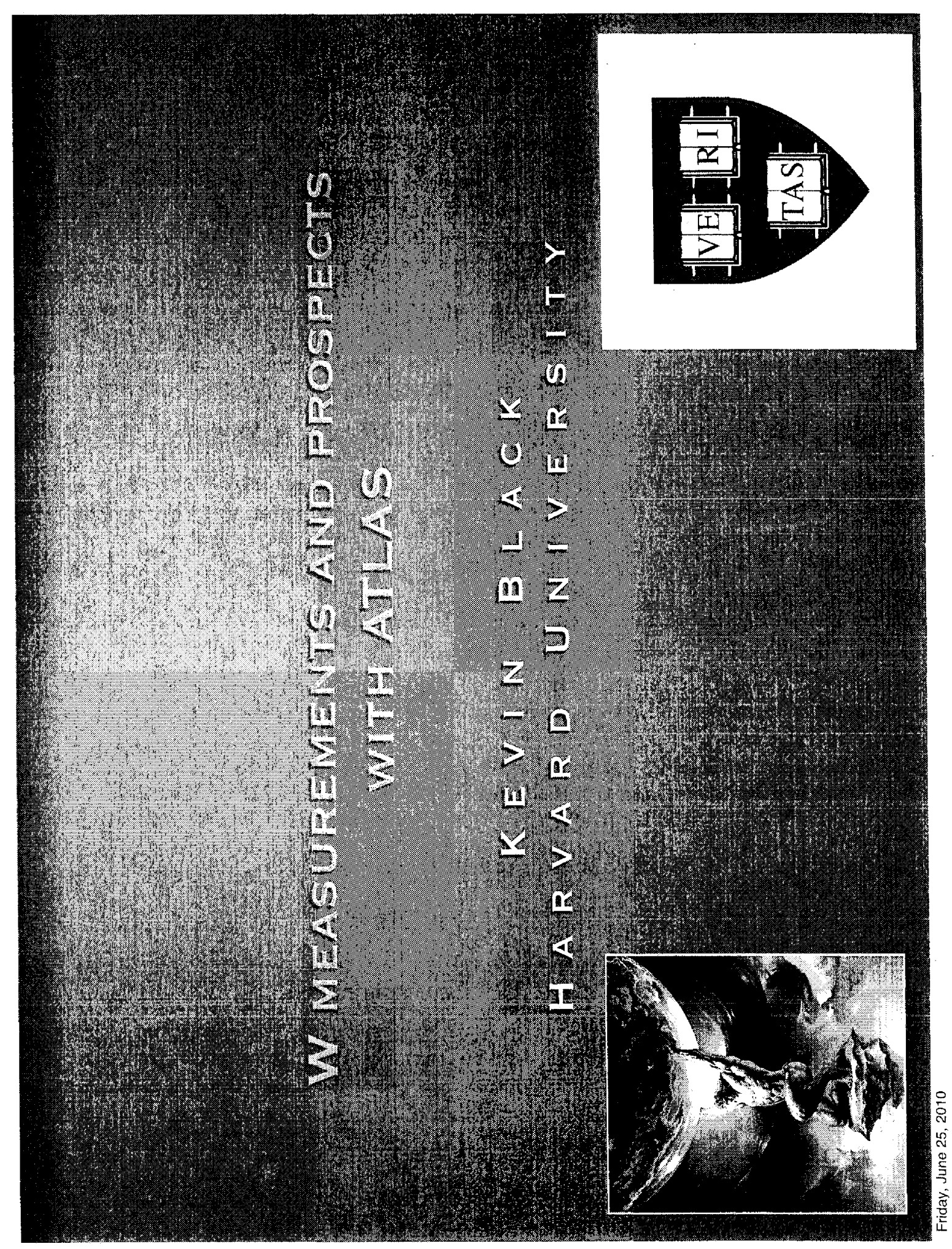




\section{OUTLINE}

㭗 Motivations

㓥 Cross-Sections at $7 \mathrm{TeV}$ and expected event yield

粰Asymmetry Measurements

宷 $\mathrm{W}$. mass prospects

勘 First W results from ATLAS 


\section{PRECISION ELECTROWEAK}

$$
\begin{gathered}
m_{W}=\left(\frac{\pi \alpha_{E M}}{\sqrt{2} G_{F}}\right)^{\frac{1}{2}} \frac{1}{\sin \theta_{W} \sqrt{1-\Delta r}} \\
f\left(m_{\text {top }}^{2}, \log m_{h}\right) \\
\Delta m_{W} \approx 0.7 \times 10^{-2} \Delta m_{t o p}
\end{gathered}
$$

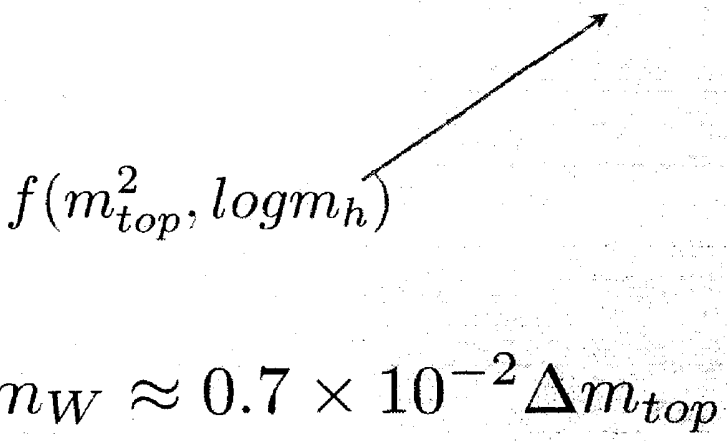

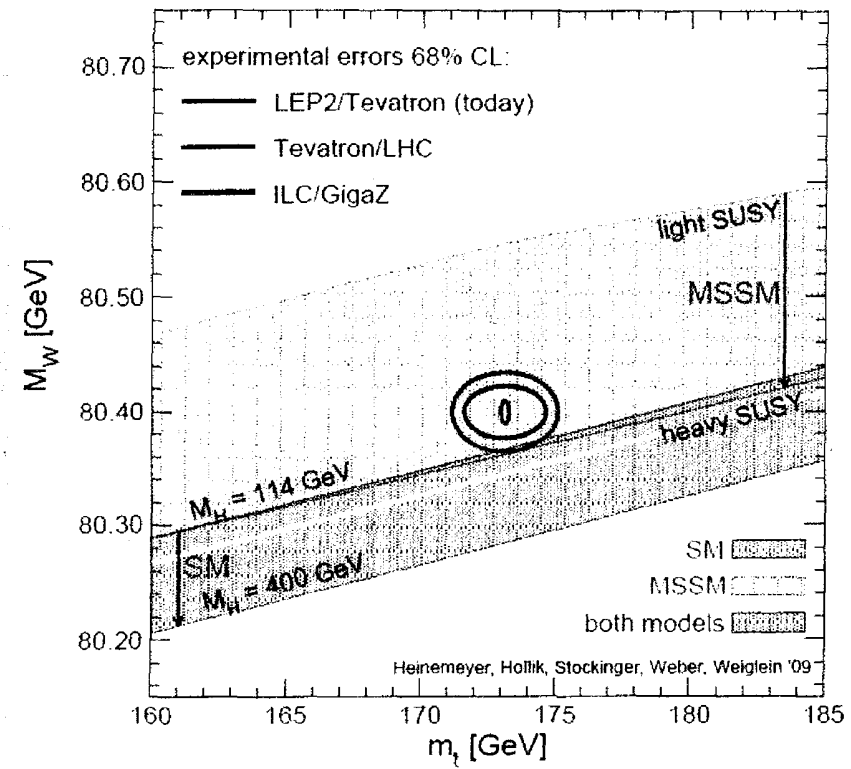

$\Delta m_{W} \approx 10 \mathrm{MeV}$ 


\section{EXPECTED \\ CROSS-SECTIONS}

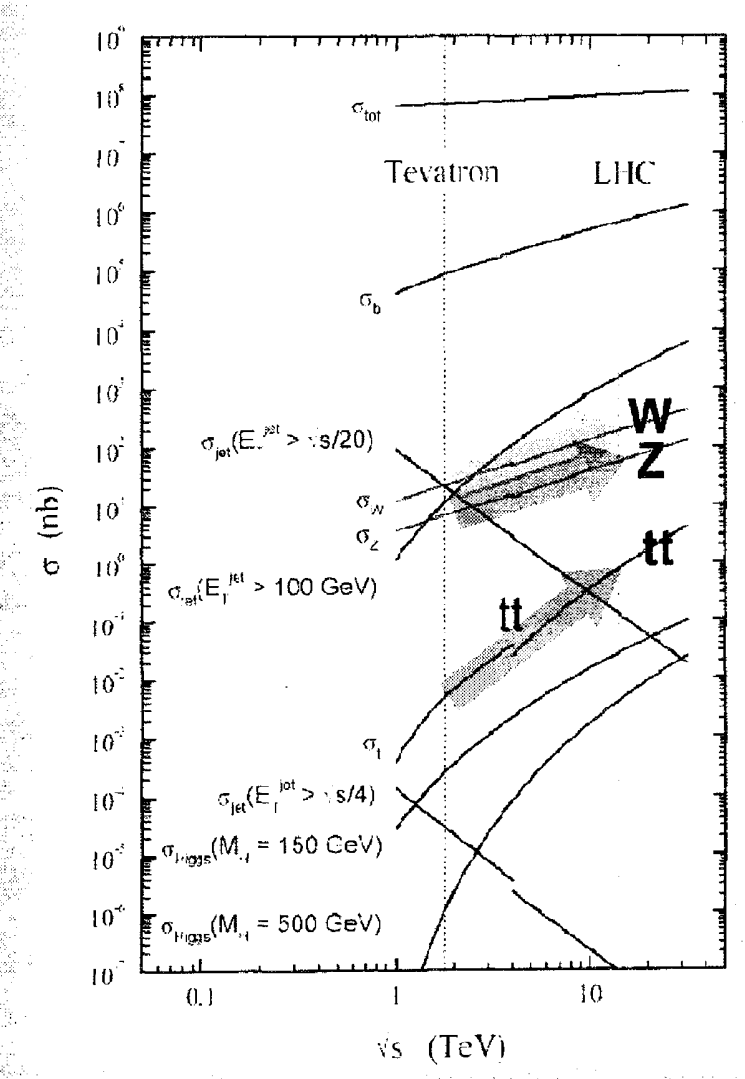

At $7 \mathrm{TeV}$

$$
\begin{gathered}
\sigma^{N N L O}(\mathrm{~W} \rightarrow \mathrm{l} v)=10.45 \mathrm{nb} \\
\text { roughly } \mathrm{x} 2 \text { at } 14 \mathrm{TeV}
\end{gathered}
$$

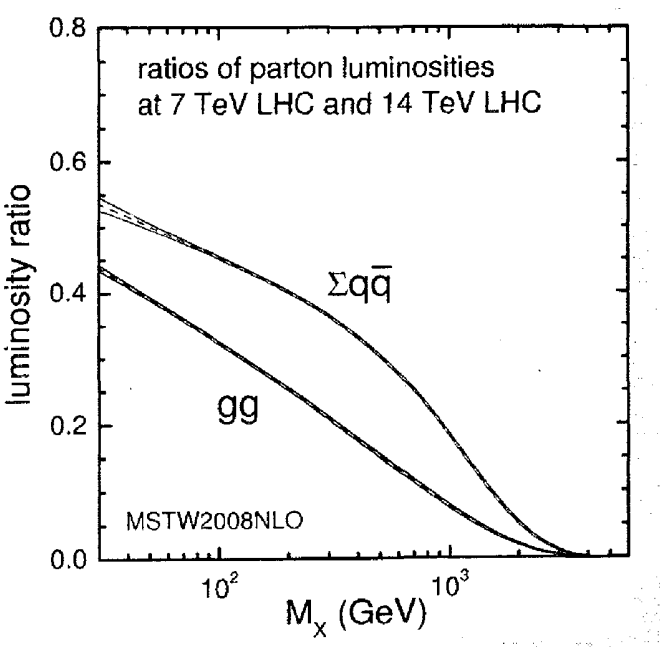




\section{CROSS-SECTION MEASUREMENTS}

宷 $\mathrm{P}_{\mathrm{T}}>25 \mathrm{GeV}$ lepton

轔 Missing Et $>25 \mathrm{GeV}$

粪 Expected uncertainty (stat+sys, no lumi):

絭 - $5 \%$ after $50 \mathrm{pb}^{-1}$

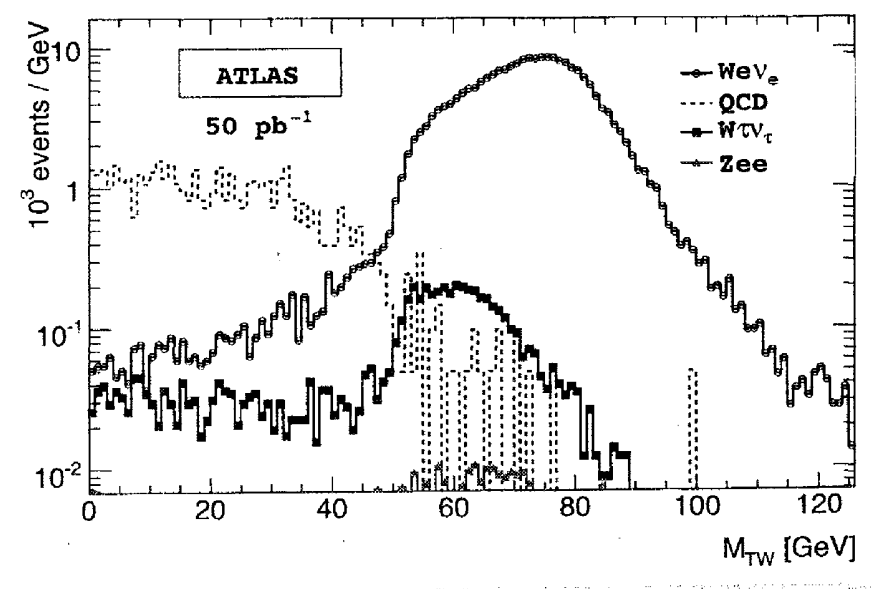

絭 $2.5 \%$ after $1 \mathrm{fb}^{-1}$

$$
\begin{aligned}
& M_{T}=\sqrt{p_{T}^{l} E_{T}^{\text {miss }}\left(1-\cos \Delta \phi\left(p_{T}^{l}, E_{T}^{m i s s}\right)\right)} \\
& \text { factor of } 2 \text { less W's at } 7 \mathrm{TeV}
\end{aligned}
$$




\section{SUMMARY}

番 LHC will offer unprecedented number of W's for a variety of studies:

㓥 Detector Commissioning

轔Precision Electroweak Physics

橉Backgrounds for 'new' physics

漛 Just getting started but excellent early results 
The Physics of $W$ and $Z$ Bosons

RIKEN BNL Research Center Workshop

June $24 / 25,2010$

\title{
Current Status and Prospects for $W$ and $Z$ Cross Section Measurements at CMS
}

\author{
Carsten Magass ${ }^{1}$ \\ (for the CMS Collaboration) \\ III. Physikalisches Institut A \\ RWTH Aachen \\ D-52056 Aachen (Germany)
}

\begin{abstract}
:
Current status and prospects for $W$ and $Z$ cross section measurements at CMS are presented. Events have been selected in electron and muon channels, and candidate $W$ and $Z$ decays have been examined. The measurements of the cross sections are in progress, and details of lepton identification, missing energy measurement, and event selection are described. Furthermore, prospects for future measurements are presented, like the measurement of the $Z$ boson rapidity distribution and the muon charge asymmetry in $W$ decays. Finally, selected studies of $W / Z$ boson production in association with jets are discussed.
\end{abstract}

CMS Physics Results Webpage:

https://twiki.cern.ch/twiki/bin/view/CMS/PublicPhysicsResults

${ }^{1}$ magass@physik.rwth-aachen. de 

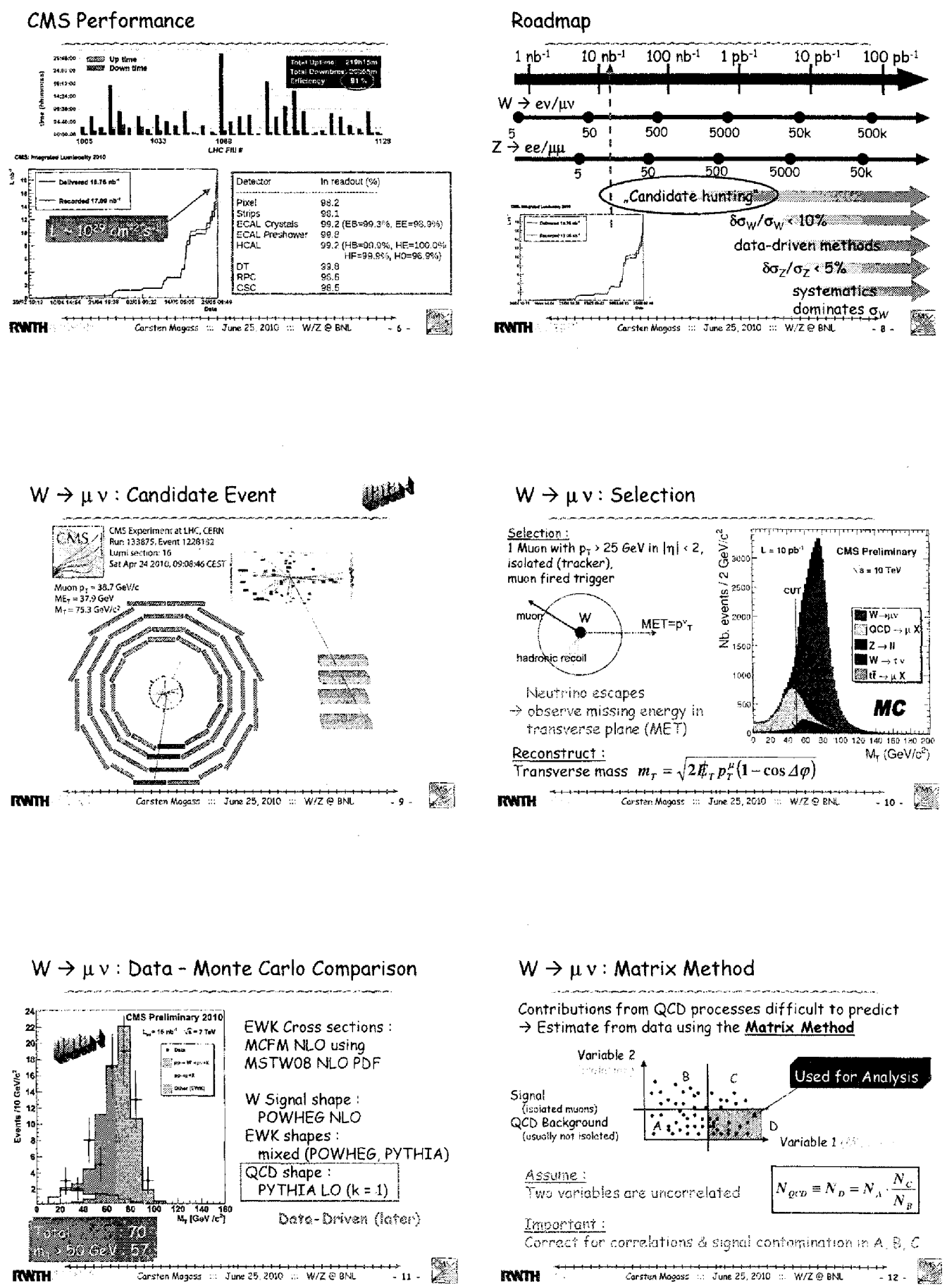

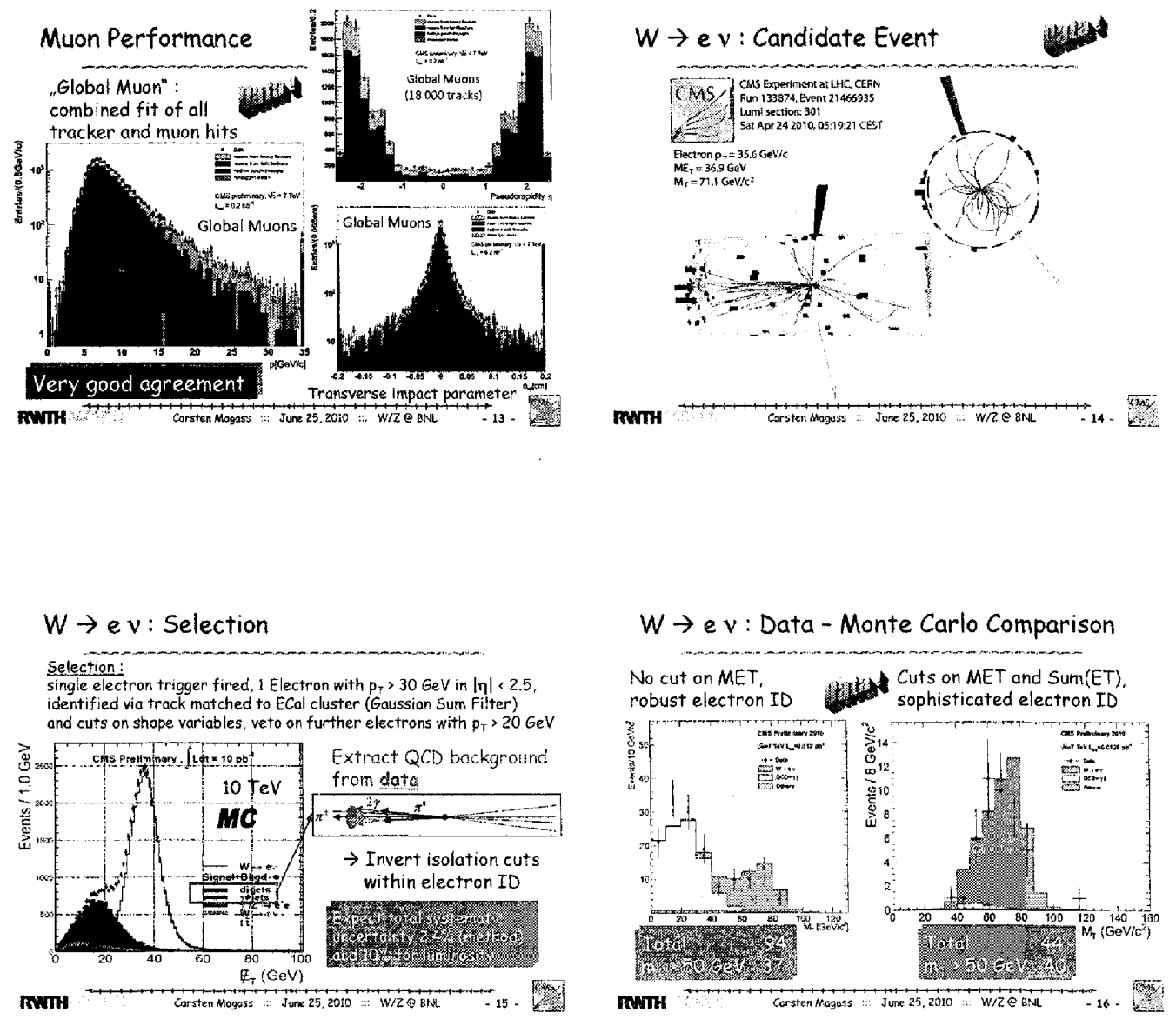

\section{$W \rightarrow$ ev : Template Method}

MET sensitive to everything (real objects, noise) in the detector

$\rightarrow$ Use Z events as „templates" to estimate MET in W events
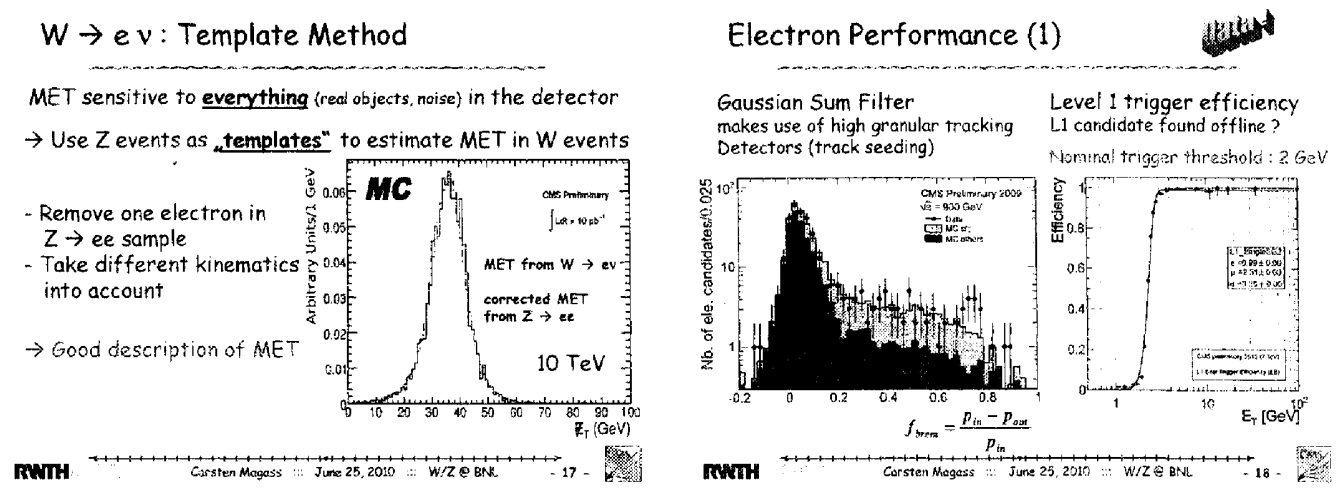

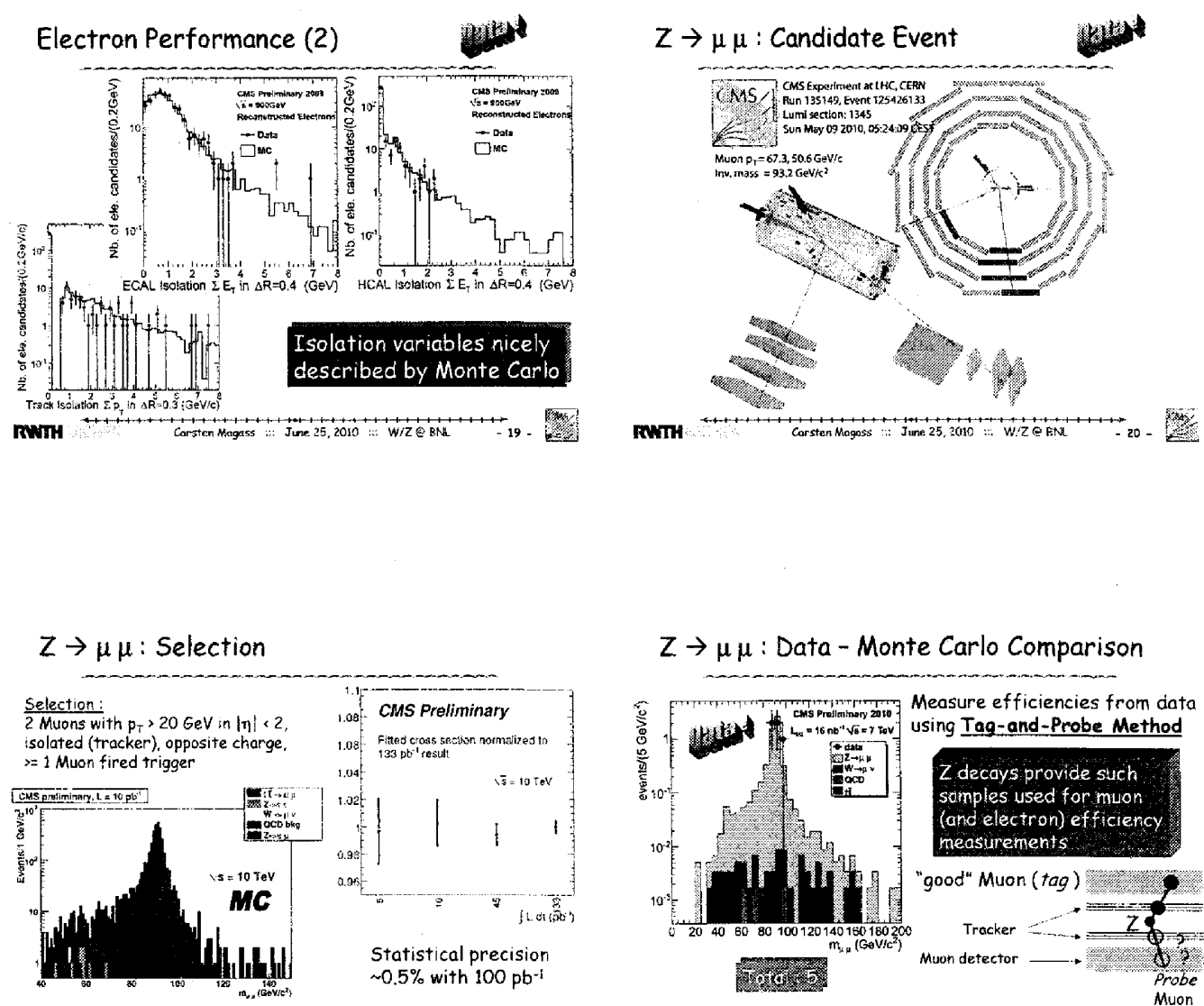

$Z \rightarrow \mu \mu$ : Data-Monte Carlo Comparison

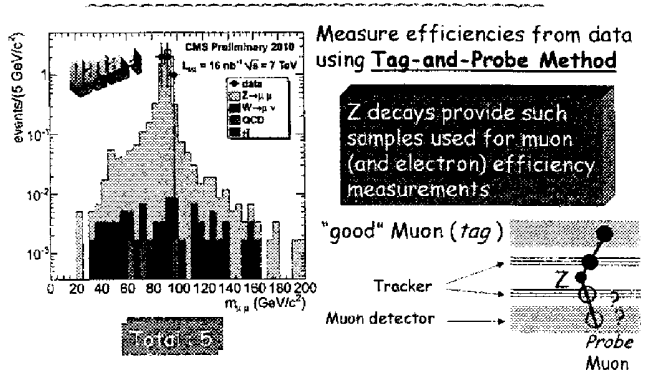

mwnt

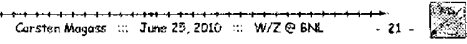

mWrH
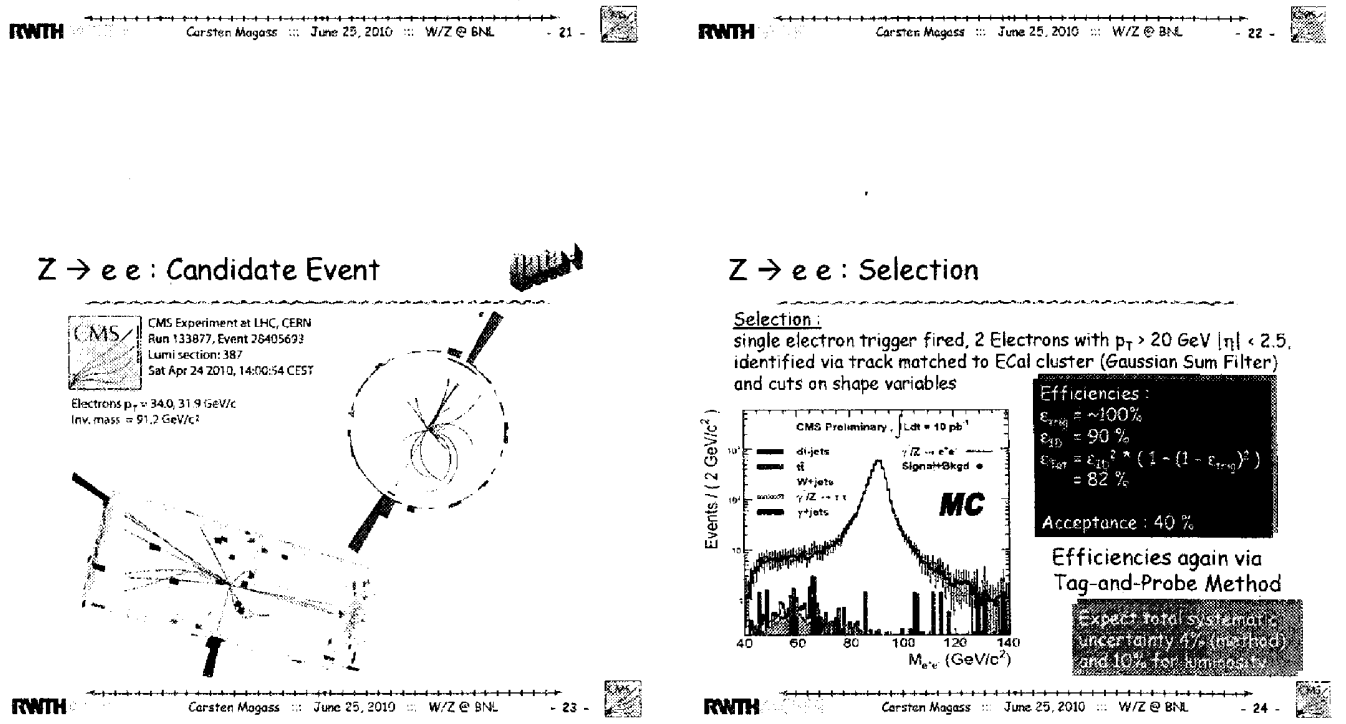


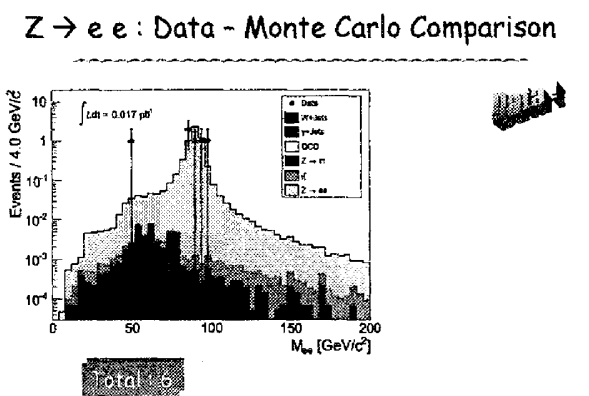

... this is just the beginning ...

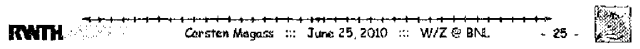

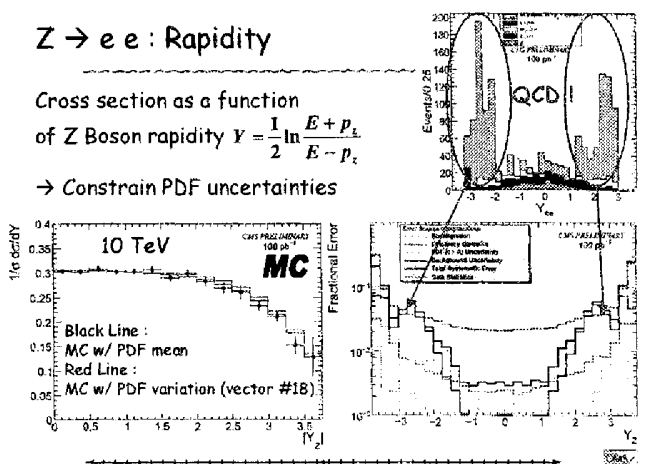

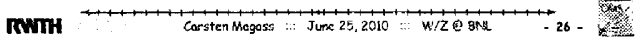
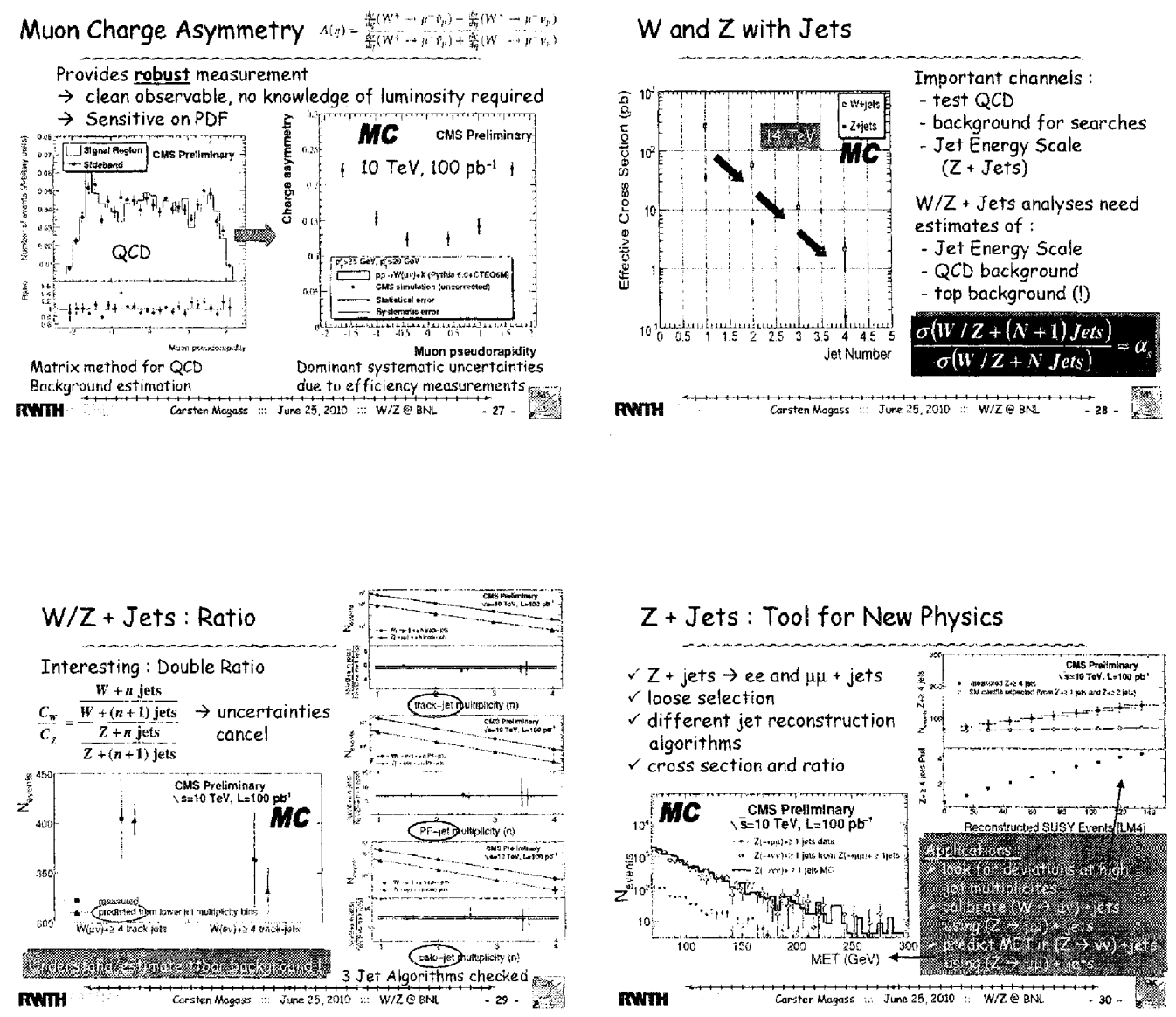

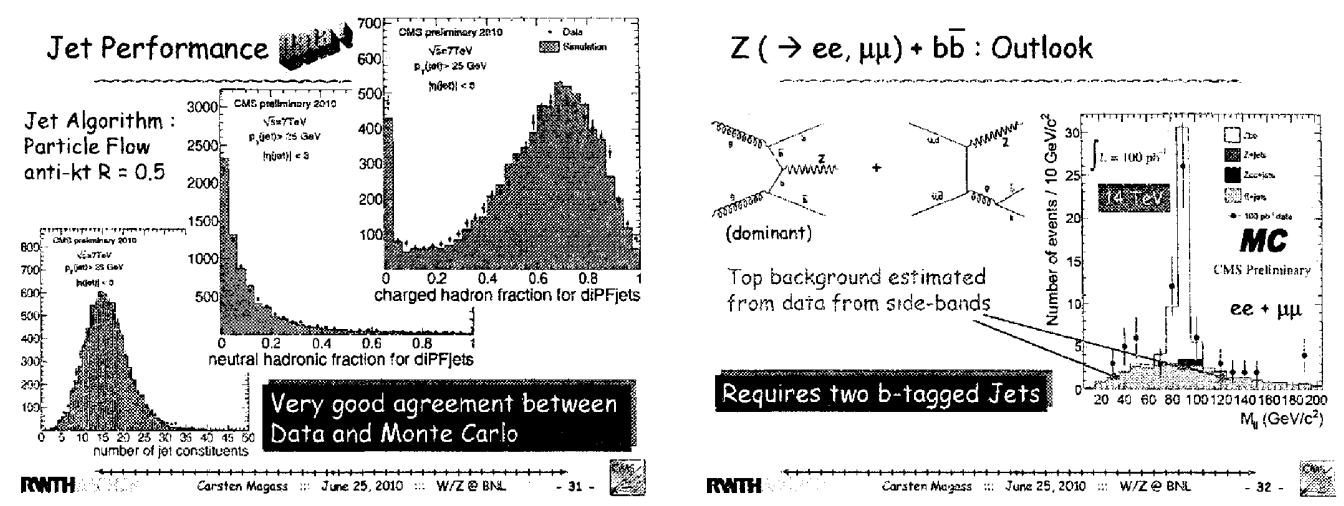

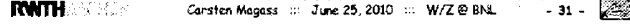
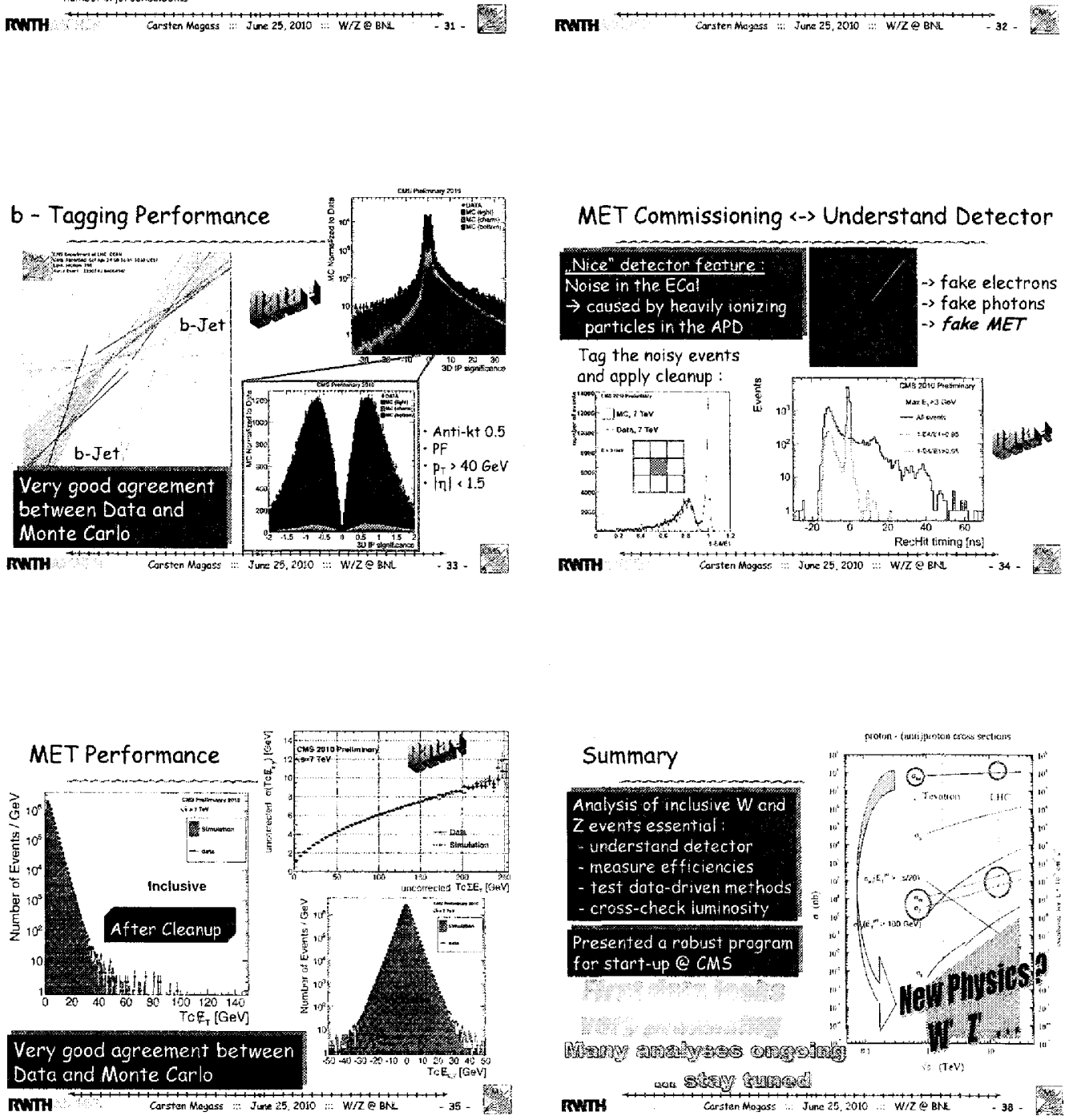


\title{
The Status of NNLO Tools for WIZ Production at Hadron Colliders
}

\author{
Frank Petriello \\ University of Wisconsin, Madison
}

The Physics of $\mathrm{W}$ and $\mathrm{Z}$ Bosons

June 25, 2010 


\section{Standard Candles}

$\sigma\left(\mu_{R}, \mu_{F}\right)=\sigma^{(0)}\left(\mu_{F}\right)+\frac{\alpha_{s}\left(\mu_{R}\right)}{\pi} \sigma^{(1)}\left(\mu_{R}, \mu_{F}\right)+\left(\frac{\alpha_{s}\left(\mu_{R}\right)}{\pi}\right)^{2} \sigma^{(2)}\left(\mu_{R}, \mu_{F}\right)$

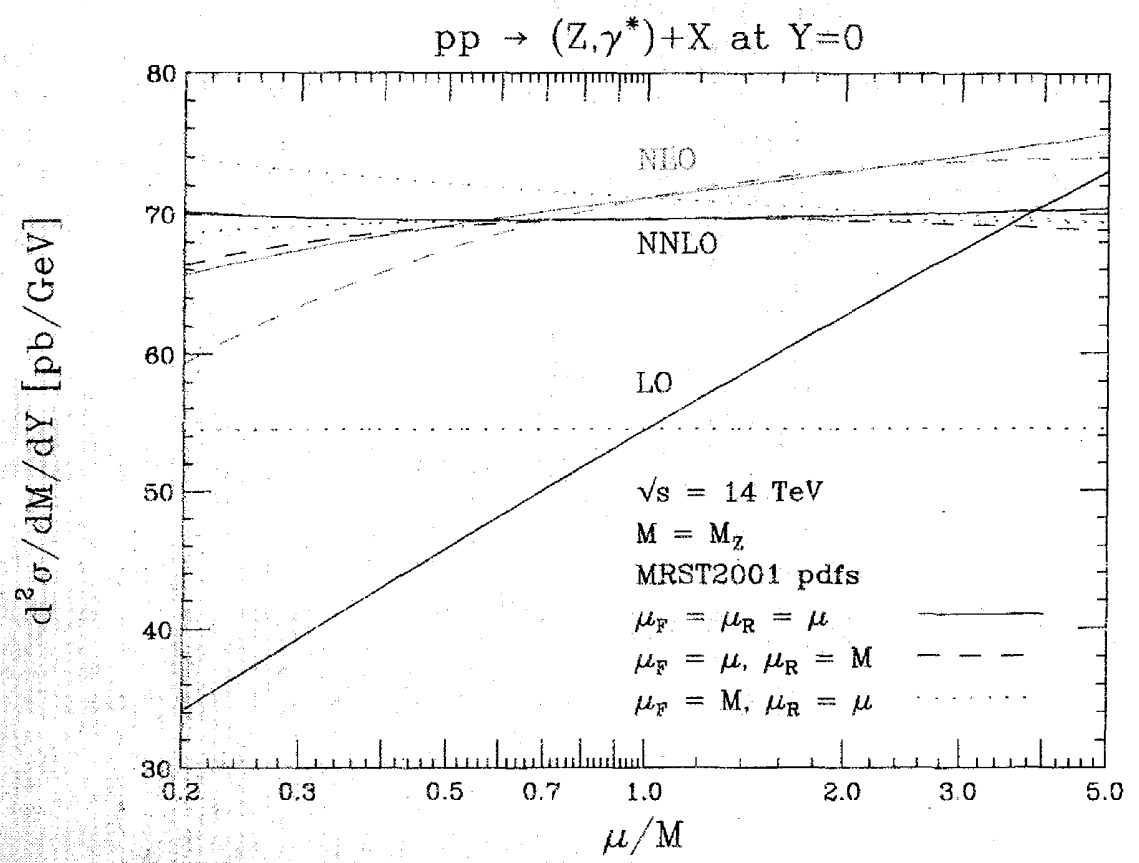

* W,Z production known through NNLO in $\mathrm{pQCD}$

¿ Residual theoretical uncertainties from scale variations $<\mathrm{r} \%$ on inclusive quantities

" "Gold-plated" observables suited to high-precision measurements

Anastasiou, Dixon, Melnikov, FP (ADMP) hep-ph/o3ı2266 


\section{$\gamma^{*} / Z$ rapidity distributions}

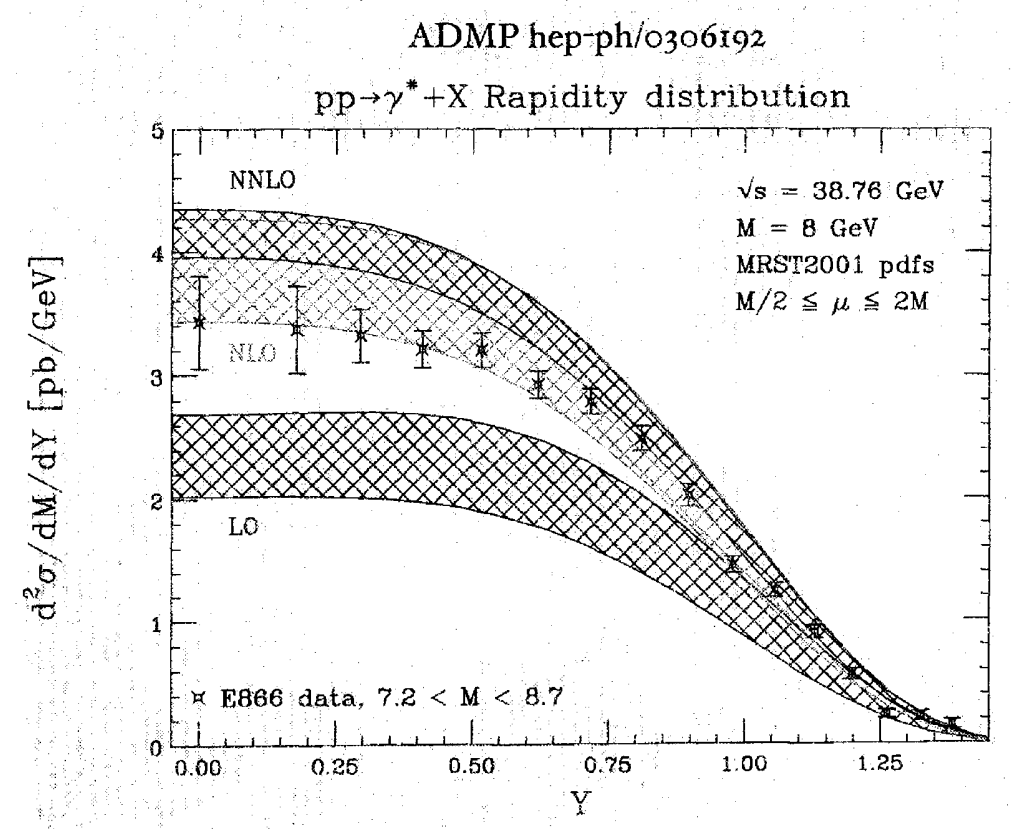

- Significant impact on fixedtarget data, quark distributions MSTW 0706.0459 , 0901.0002

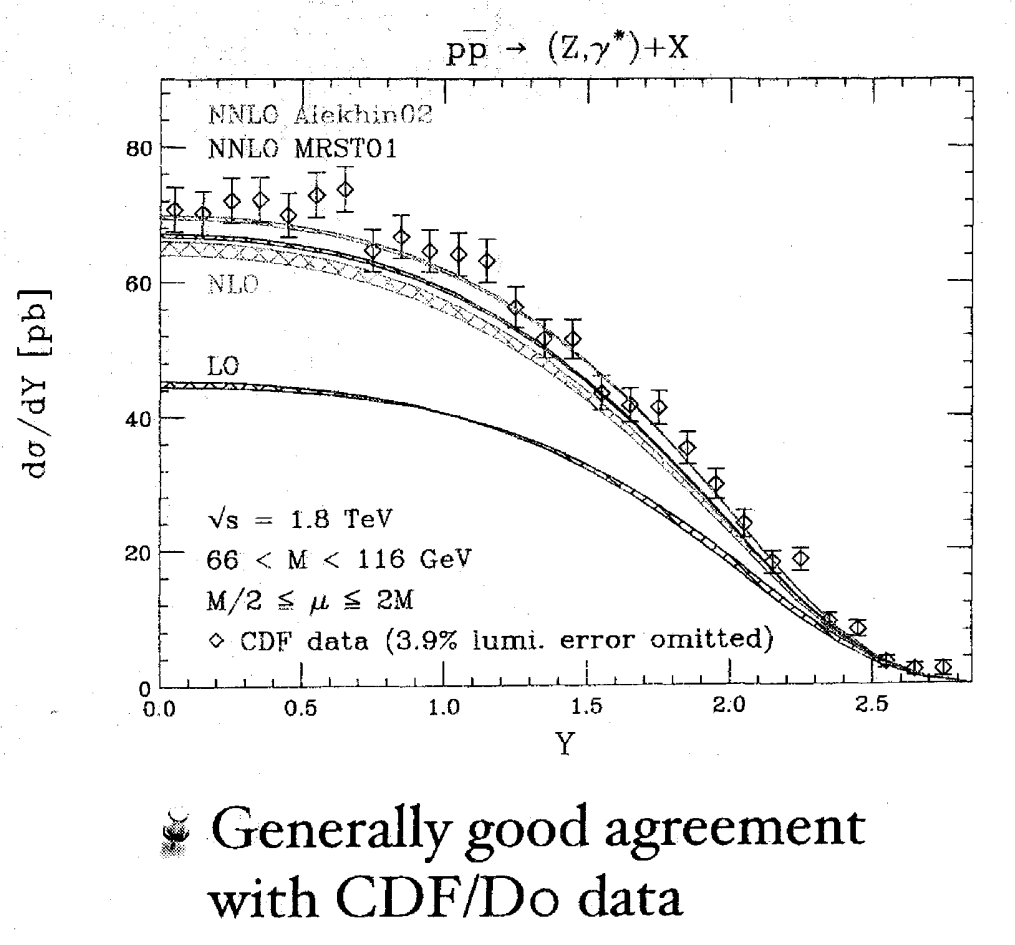

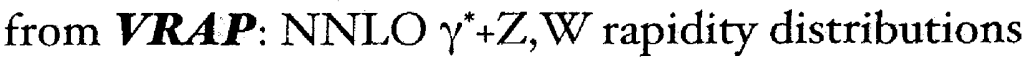




\section{Spin correlations}

$$
\sigma=\frac{N}{\epsilon \times \mathcal{L} \times A} \longrightarrow \begin{aligned}
& \text { Measure leptons, not } \mathrm{W} / \mathrm{Z} \text {, with cuts } \\
& \text { imposed } \Rightarrow \text { spin correlations between } \\
& \text { production, decay }
\end{aligned}
$$

- Cut 1: $\quad p_{T}^{e}>20 \mathrm{GeV}, \quad\left|\eta^{e}\right|<2.5, \quad E_{T}>20 \mathrm{GeV}$;

- Cut 2: $\quad p_{T}^{e}>40 \mathrm{GeV},\left|\eta^{\varepsilon}\right|<2.5, \quad E_{T}>20 \mathrm{GeV}$.

\begin{tabular}{l|ccc|ccc}
\hline & \multicolumn{3}{|c|}{ Tevatron } & \multicolumn{3}{c}{ LHC } \\
& LO & NLO & MCGNLO & LO & NLO & MCoNLO \\
\hline Cut 1 & 0.409 & 0.385 & 0.383 & 0.524 & 0.477 & 0.485 \\
Cut 1, no spin & 0.413 & 0.394 & 0.394 & 0.553 & 0.510 & 0.515 \\
\hline Cut 2 & 0.356 & 0.340 & 0.336 & 0.058 & 0.129 & 0.133 \\
Cut 2, no spin & 0.389 & 0.374 & 0.370 & 0.075 & 0.150 & 0.157 \\
\hline
\end{tabular}

Essential: fully differential NNLO with spin correlations, $\gamma / Z$ interference 


\section{FEWZ}

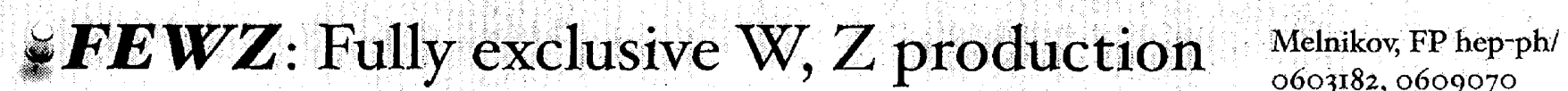

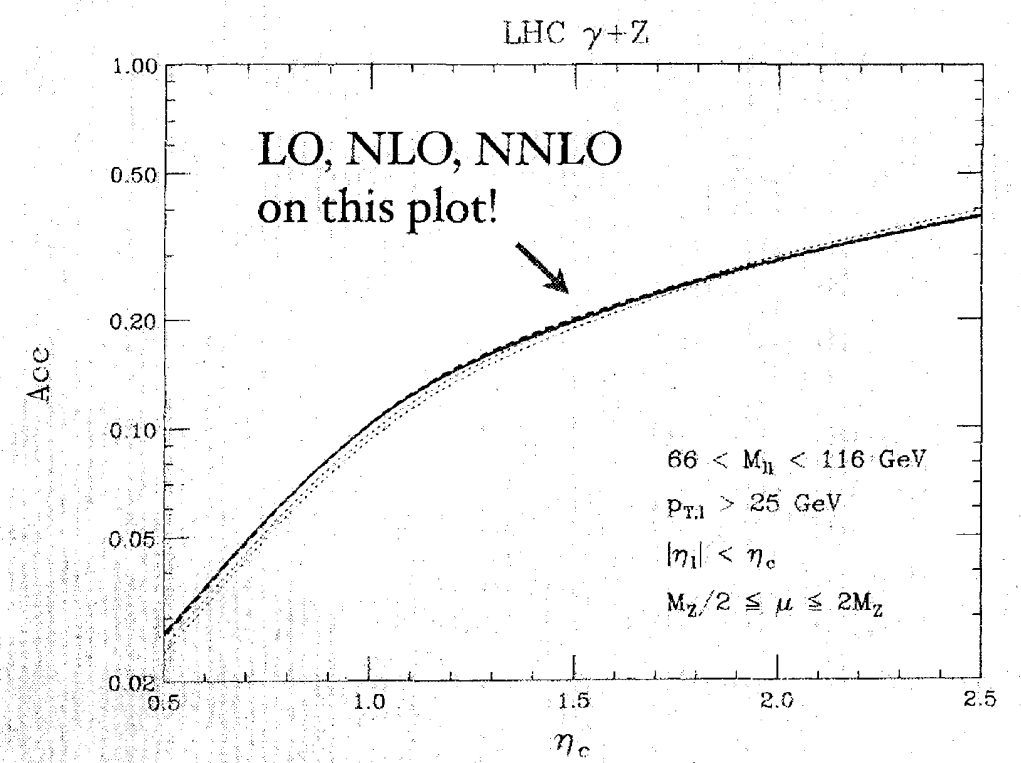

छGenerally, percent-level predictions for acceptances

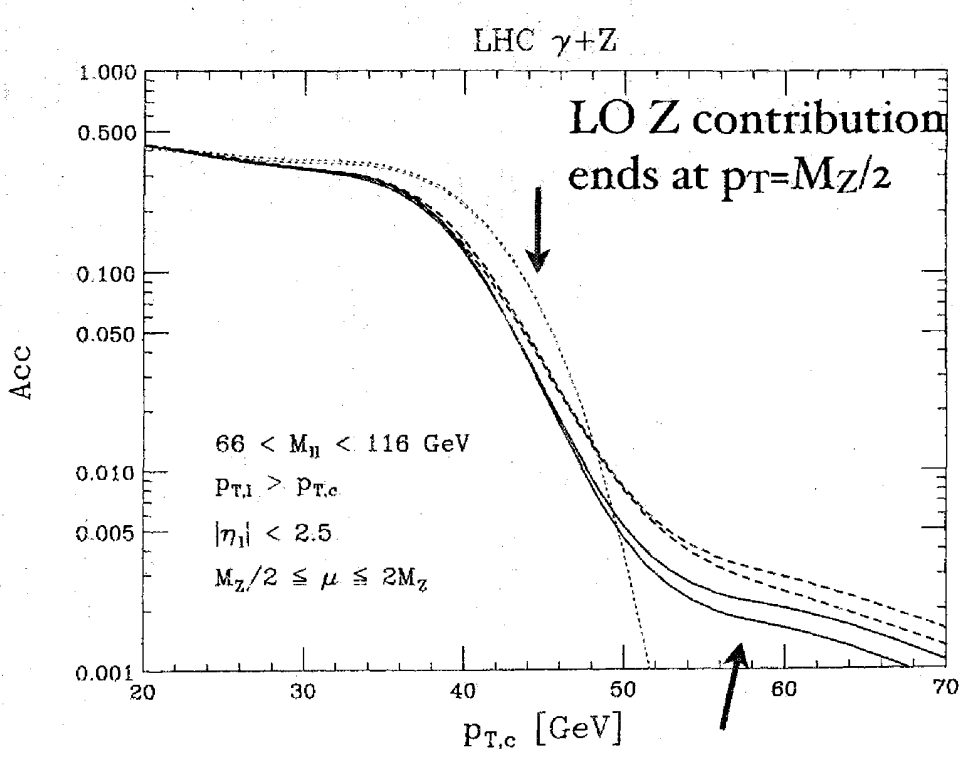

Need $V+$ jet at NNLO for this region Boughezal, Gehrmannde Ridder, Ritzmann Ioor.2396 


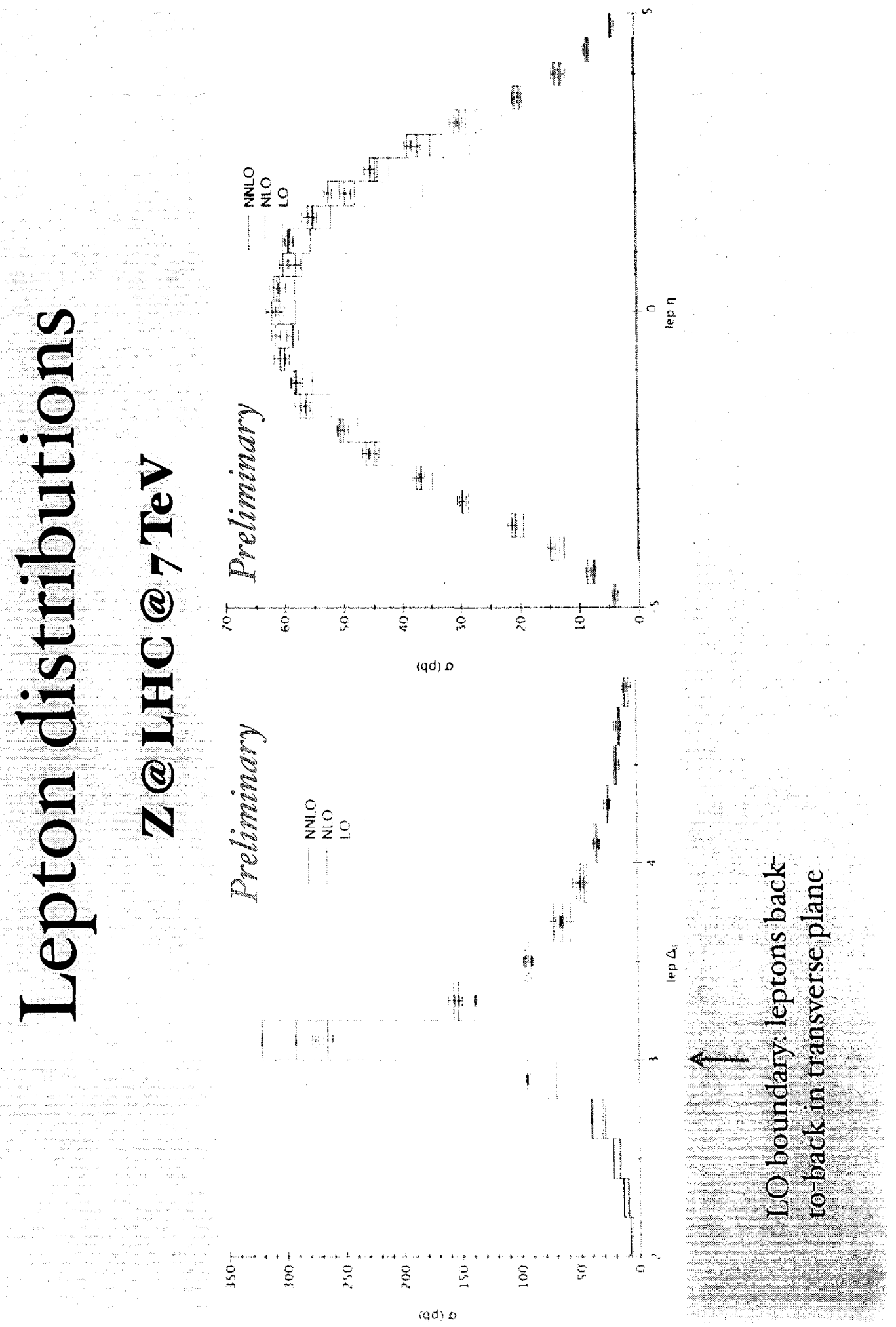




\section{DYNNLO}

- Alternative subtraction approach to handling

IR singularities at NNLO Catani, Ciert, Ferrera, de Florian, Grazzini o903.2220

ڤ
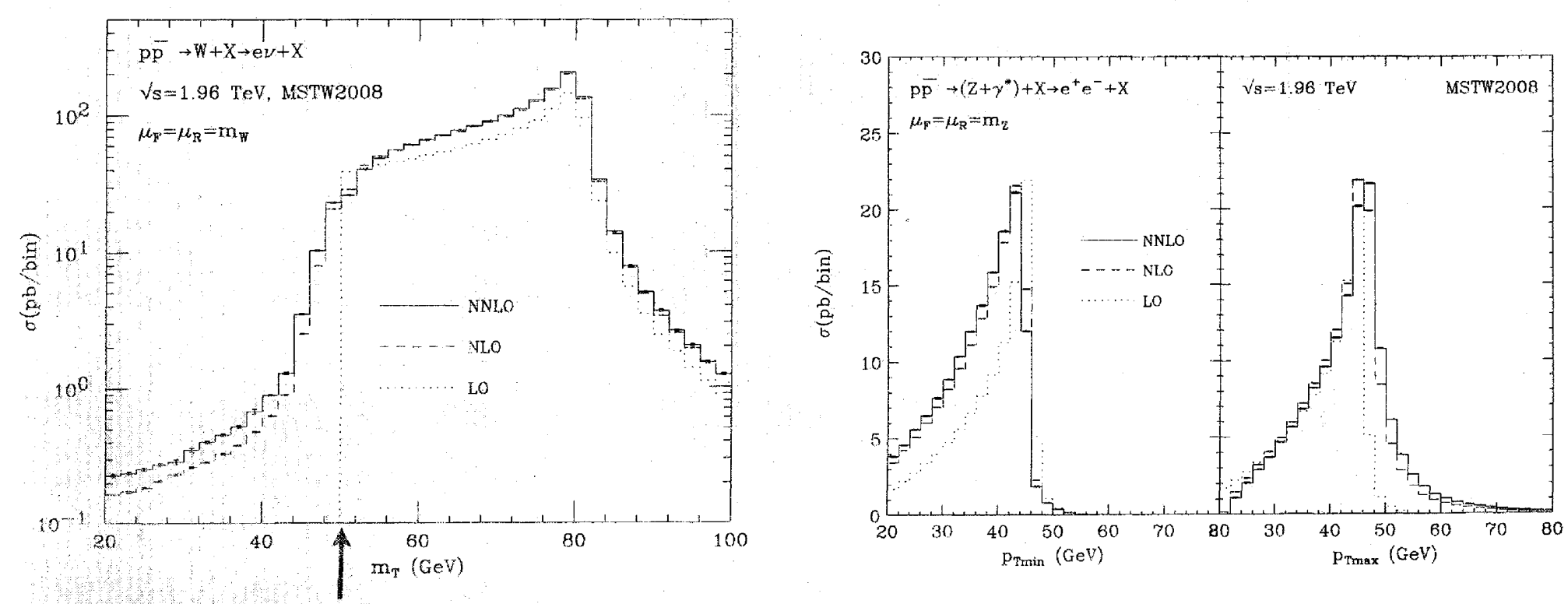

LO boundary created by $\mathrm{pT}^{\text {miss }}>25 \mathrm{GeV}$ 


\title{
The Physics of $\mathrm{W}$ and $\mathrm{Z}$ Bosons
}

\author{
June 24-25, 2010 \\ Registered Participants
}

\begin{tabular}{|c|c|c|}
\hline Name & Affiliation & E-Mail Address \\
\hline Yasuyuki Akiba & RIKEN & akiba@bnl.gov \\
\hline Leandro Almeida & $\mathrm{BNL}$ & leandro.g.almeida@gmail.com \\
\hline Jan Balewski & MIT & balewski@mit.edu \\
\hline Kenneth Barish & UC Riverside & Kenneth.Barish@ucr.edu \\
\hline Ulrich Baur & SUNY@Buffalo & baur@buffalo.edu \\
\hline Alexander Bazilevsky & BNL & shura@bnl.gov \\
\hline Andrew Beckwith & Amer Inst of Beam Energy Propulsion & beckwith@aibep.org \\
\hline Kevin Black & Harvard Univ & kblack@fas.harvard.edu \\
\hline Radja Boughezal & Univ of Zurich, Switzerland & radja.boughezal@physik.uzh.ch \\
\hline Kieran Boyle & RBRC & kboyle@bnl.gov \\
\hline Ning Chen & Stony Brook Univ & chen@max2.physics.sunysb.edu \\
\hline Mickey Chiu & $\mathrm{BNL}$ & chiu@bnl.gov \\
\hline Michael Daugherity & Abilene Christian Univ & mike.daugherity@acu.edu \\
\hline Hooman Davoudiasl & $\mathrm{BNL}$ & hooman@bnl.gov \\
\hline Sally Dawson & BNL & dawson@bnl.gov \\
\hline Abhay Deshpande & Stony Brook Univ & abhay.deshpande@stonybrook.edu \\
\hline Chee Sheng Fong & Stony Brook Univ & fong@insti.physics.sunysb.edu \\
\hline Yoshi Fukao & RIKEN & fukao@riken.jp \\
\hline Ciprian Gal & Stony Brook Univ & chip.ef@gmail.com \\
\hline Thomas Gehrmann & Univ of Zurich & thomas.gehrmann@physik.unizh.ch \\
\hline Yuji Goto & RIKEN & goto@bnl.gov \\
\hline Wlodek Guryn & BNL & guryn@bnl.gov \\
\hline Marco Guzzi & Southern Methodist Univ & mguzzi@physics.smu.edu \\
\hline John Haggerty & $\mathrm{BNL}$ & haggerty@bnl.gov \\
\hline Prerit Jaiswal & Stony Brook Univ & prerit.jaiswal@gmail.com \\
\hline Zhongbo Kang & RBRC & zkang@bnl.gov \\
\hline David Kawall & RBRC / Univ of MA & kawall@bnl.gov \\
\hline Jennifer Kile & $\mathrm{BNL}$ & jenkile@quark.phy.bnl.gov \\
\hline William Kilgore & $\mathrm{BNL}$ & kilgore@bnl.gov \\
\hline Ashutosh Kotwal & Duke Univ & kotwal@fnal.gov \\
\hline Hye-Sung Lee & $\mathrm{BNL}$ & hlee@bnl.gov \\
\hline Sookhyun Lee & Stony Brook Univ & shlee@bnl.gov \\
\hline Carsten Magass & RWTH Aachen & magass@physik.rwth-aachen.de \\
\hline William Marciano & BNL & marciano@bnl.gov \\
\hline Thomas McElmurry & $\mathrm{BNL}$ & tmcelmurry@quark.phy.bnl.gov \\
\hline David Morrison & $\mathrm{BNL}$ & morrison@bnl.gov \\
\hline Pavel Nadolsky & Southern Methodist Univ & nadolsky@physics.smu.edu \\
\hline Shigemi Ohta & IPNS/KEK+SOKENDAI+RBRC & shigemi.ohta@kek.jp \\
\hline Kensuke Okada & RBRC & okada@bnl.gov \\
\hline Fredrick Olness & Southern Methodist Univ & olness@smu.edu \\
\hline Kwangwoo Park & Southern Methodist Univ & kwpark@mail.smu.edu \\
\hline Zohreh Parsa & BNL & parsa@bnl.gov \\
\hline
\end{tabular}




\begin{tabular}{|l|l|l|}
\hline Abid Patwa & BNL & abid@fnal.gov \\
\hline Michael Peskin & SLAC Natl Accelerator Lab & mpeskin@slac.stanford.edu \\
\hline Francis Petriello & Univ of Wisconsin & frankjp@physics.wisc.edu \\
\hline Marc-Andre Pleier & BNL & mpleier@bnl.gov \\
\hline Jianwei Qiu & BNL & jqiu@bnl.gov \\
\hline Guy Roche & LPC-Clermont France & roche@in2p3.fr \\
\hline Renata Rodrigues & CBPF & renatar@fnal.gov \\
\hline Andreas Scharf & SUNY at Buffalo & abscharf@buffalo.edu \\
\hline Joseph Seele & MIT & seelej@mit.edu \\
\hline Amarjit Soni & BNL & soni@bnl.gov \\
\hline David South & TUDortmund & david.south@,desy.de \\
\hline Jan Stark & LPSC Grenoble & stark@in2p3.fr \\
\hline Justin Stevens & Indiana Univ & stevens4@indiana.edu \\
\hline Christian Sturm & BNL & sturm@bnl.gov \\
\hline Bernd Surrow & MIT & surrow@mit.edu \\
\hline Swadhin Taneja & Stony Brook Univ & swadhin.tanega@gmail.com \\
\hline Michael Tannenbaum & BNL & mjt@bnl.gov \\
\hline Rusty Towell & Abilene Christian Univ & rusty.towell@acu.edu \\
\hline Kathryn Tschann-Grimm & Stony Brook Univ & kgrimm@grad.physics.sunysb.edu \\
\hline Alessandro Vicini & Univ of Milano & alessandro.vicini@mi.infn.it \\
\hline Doreen Wackeroth & SUNY@ Buffalo & dow@ubpheno.physics.buffalo.edu \\
\hline Jan Winter & Fermilab & jwinter@fnal.gov \\
\hline Scott Yost & The Citadel & scott.yost@citadel.edu \\
\hline C.-P. Yuan & Michigan State Univ & yuan@pa.msu.edu \\
\hline Feng Yuan & LBNL & fyuan@lbl.gov \\
\hline Guang-Da Zhao & Peking University & ktchao@pku.edu.cn \\
\hline JunjieZhu & Univ of Michigan & junjii@umich.edu \\
\hline & & \\
\hline
\end{tabular}




\section{The Physics of $\mathbf{W}$ and $Z$ Bosons}

Brookhaven National Laboratory

Physics Department Large Seminar Room

June $24-25,2010$

\section{Thursday 24 June 2010}

09:00->10:40 Session I [ Convener: Jianwei Qiu (BNL) ]

09:00 Welcome Remarks

Nick Samios (RBRC)

09:10 First Measurement of $W+/ W$ - Boson Production at STAR In Polarized pp Collisions at RHIC

Jan Balewski (MIT)

09:50 First Observations at PHENIX of W Production From

Polarized pp Collisions at RHIC

David Kawall (U. of Massachusetts,

Amherst)

$10: 30$

Coffee

10:50->12:10 Session II [ Convener: C.-P. Yuan (MSU) ]

10:50 QCD Resummation on A_L of W P_T Distributions and PDFs

Pavel Nadolsky (Southem

Methodist U.)

11:30 W Mass Measurements

Alessandro Vicini (U. of

Milan)

12:10

Lunch at Berkner Hall

\section{3:30->15:30 Session III [ Convener: Abid Patwa (BNL)]}

13:30 W/Z Physics from HERA

David South (DESY)

14:10 W Mass Measurements at DO

Junjie Zhu (U. of Michigan)

14:50 W Mass Measurements at CDF

Ashutosh Kotwal (Duke U.)

$15: 30$

Coffee

\section{5:50->17:50 Session IV [ Convener: Kensuke Okada (BNL/RIKEN)]}

15:50 Monte Carlo Modeling Issues for W Measurements

16:30 Theoretical Issues in Monte Carlo Modelling for W Production

17:10 W Boson Physics and Global Analysis of PDFs
Jan Stark (LPSC, Grenoble) Jan Winter (Fermilab)

C. -P. Yuan (Michigan State U.) 


\section{Friday 25 June 2010}

09:00->10:20 Session V [ Convener: Abhay Deshpande (Stony Brook U)]

09:00 Future Prospects of the STAR W Program in Polarized pp Collisions at RHIC

Joe Seele $(M / T)$

09:40 Prospects of the Forward W Measurement in Polarized pp Collisions at the RHIC-PHENIX Experiment

10:50->12:10 Session VI [ Convener: Frank Petriello (Wisconsin)]

10:50 A_N of W Production in Polarized pp Collisions

11:30 Precision Measurement of the W Mass and New Physics

12:10

Lunch at Berkner Hall
Zhong-Bo Kang (RBRC) Ulrich Baur (U. at Buffalo)

14:00->16:00 Session VII [ Convener: Sally Dawson (BNL)]

14:00 W Measurements and Prospects at ATLAS

Kevin Black (Harvard) Current Status and Prospects for W \& Z Cross Section Measurements at CMS

15:20 The Status of NNLO Tools for W/Z Boson Production at Hadron Colliders 


\section{Additional RIKEN BNL Research Center Proceedings:}

Volume 98 - Saturation, the Color Glass Condensate and the Glasma: What Have we Learned from RHIC?, BNL, May 10-12, 2010 - BNL-94271-2010

Volume 97 - RBRC Scientific Review Committee Meeting, October 21-22, 2009 - BNL-90674-2009

Volume 96 - P-and CP-Odd Effects in Hot and Dense Matter, April 26-30, 2010 - BNL-94237-2010

Volume 95 - Progress in High-pT Physics at RHIC, March 17-19, 2010 - BNL-94214-2010

Volume 94 - Summer Program on Nucleon Spin Physics at LBL, June 1-12, 2009

Volume 93

- PHENIX Spinfest School 2009 at BNL - July 1-31, 2009. BNL-90343-2009

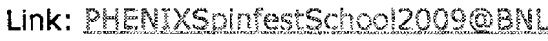

Volume 92

Volume 91

Volume 90

Volume 89

Volume 88

Volume 87

Volume 86

Volume 85

Volume 84

Volume 83

Volume 82

Volume 81

Volume 80

Volume 79

Volume 78

Volume 77

Volume 76

Volume 75

volume 74

Volume 73

Volume 72

Volume 71

Volume 70

Volume 69

Volume 68

Volume 67

volume 66

volume 65

Volume 64

Volume 63

Volume 62

Volume 61

Volume 60

Volume 59

Volume 58

Volume 57
- PKU-RBRC Workshop on Transverse Spin Physics, June 30-July 4, 2008, Beijing, China, BNL-81685-2008

- RBRC Scientific Review Committee Meeting, November 17-18, 2008 - BNL-81556-2008

- PHENIX Spinfest School 2008 at BNL, August 4-8, 2008 - BNL-81478-2008

- Understanding QGP through Spectral Functions and Euclidean Correlators, April 23-25, 2008 - BNL-81.3182008

- Hydrodynamics in Heavy Ion Collisions and QCD Equation of State, April 21-22, 2008 - BNL-81307-2008

- RBRC Scientific Review Committee Meeting, November 5-6, 2007 - BNL-79570-2007

- Global Analysis of Polarized Parton Distributions in the RHIC Era, October 8, 2007 - BNL-79457-2007

- Parity-Violating Spin Asymmetries at RHIC-BNL, April 26-27, 2007 - BNL-79146-2007

- Domain Wall Fermions at Ten Years, March 15-17, 2007 - BNL 77857-2007

- QCD in Extreme Conditions, July 31-August 2, 2006 - BNL-76933-2006

- RHIC Physics in the Context of the Standard Model, June 18-23, 2006 - BNL-76863-2006

- Parton Orbital Angular Momentum (Joint RBRC/University of New Mexico Workshop) February 24-26, 2006 BNL-75937-2006

- Can We Discover the QCD Critical Point at RHIC?, March 9-10, 2006 - BNL-75692-2006

- Strangeness in Collisions, February 16-17, 2006 - BNL-79763-2008

- Heavy Flavor Productions and Hot/Dense Quark Matter, Dec 12-14, 2005 - BNL-76915-2006

- RBRC Scientific Review Committee Meeting - BNL-52649-2005

- Odderon Searches at RHIC, September 27-29, 2005 - BNL-75092-2005

- Single Spin Asymmetries, June 1-3, 2005 - BNL-74717-2005

- RBRC QCDOC Computer Dedication and Symposium on RBRC QCDOC, May 26, 2005 - BNL-74813-2005

- Jet Correlations at RHIC, March 10-11, 2005 - BNL-73910-2005

- RHIC Spin Collaboration Meetings XXXI(January 14, 2005), XXXII (February 10, 2005), XXXIII (March 11, 2005) - BNL-73866-2005

- Classical and Quantum Aspects of the Color Glass Condensate - BNL-73793-2005

- Strongly Coupled Plasmas: Electromagnetic, Nuclear \& Atomic - BNL-73867-2005

- RBRC Scientific Review Committee - BNL-73546-2004

- Workshop on the Physics Programme of the RBRC and UKQCD QCDOC Machines - BNL-73604-2004

- High Performance Computing with BlueGene/L and QCDOC Architectures

- RHIC Spin Collaboration Meeting XXIX, October 8-9, 2004, Torino Italy - BNL-73534-2004

- RHIC Spin Collaboration Meetings XXVII (July 22, 2004), XXVIII (September 2, 2004), XXX (December 6, 2004) - BNL-73506-2004

- Theory Summer Program on RHIC Physics - BNL-73263-2004

- RHIC Spin Collaboration Meetings XXIV (May 21, 2004), XXV (May 27, 2004), XXVI (June 1, 2004) - BNL72397-2004

- New Discoveries at RHIC, May 14-15, 2004 - BNL- 72391-2004

- RIKEN-TODAI Mini Workshop on "Topics in Hadron Physics at RHIC", March 23-24, 2004 - BNL-72336-2004

- Lattice QCD at Finite Temperature and Density - BNL-72083-2004

- RHIC Spin Collaboration Meeting XXI (January 22, 2004), XXII (February 27, 2004), XXIII (March 19, 2004)BNL-72382-2004

- RHIC Spin Collaboration Meeting XX - BNL-71900-2004

- High pt Physics at RHIC, December 2-6, 2003 - BNL-72069-2004 


\section{Additional RIKEN BNL Research Center Proceedings:}

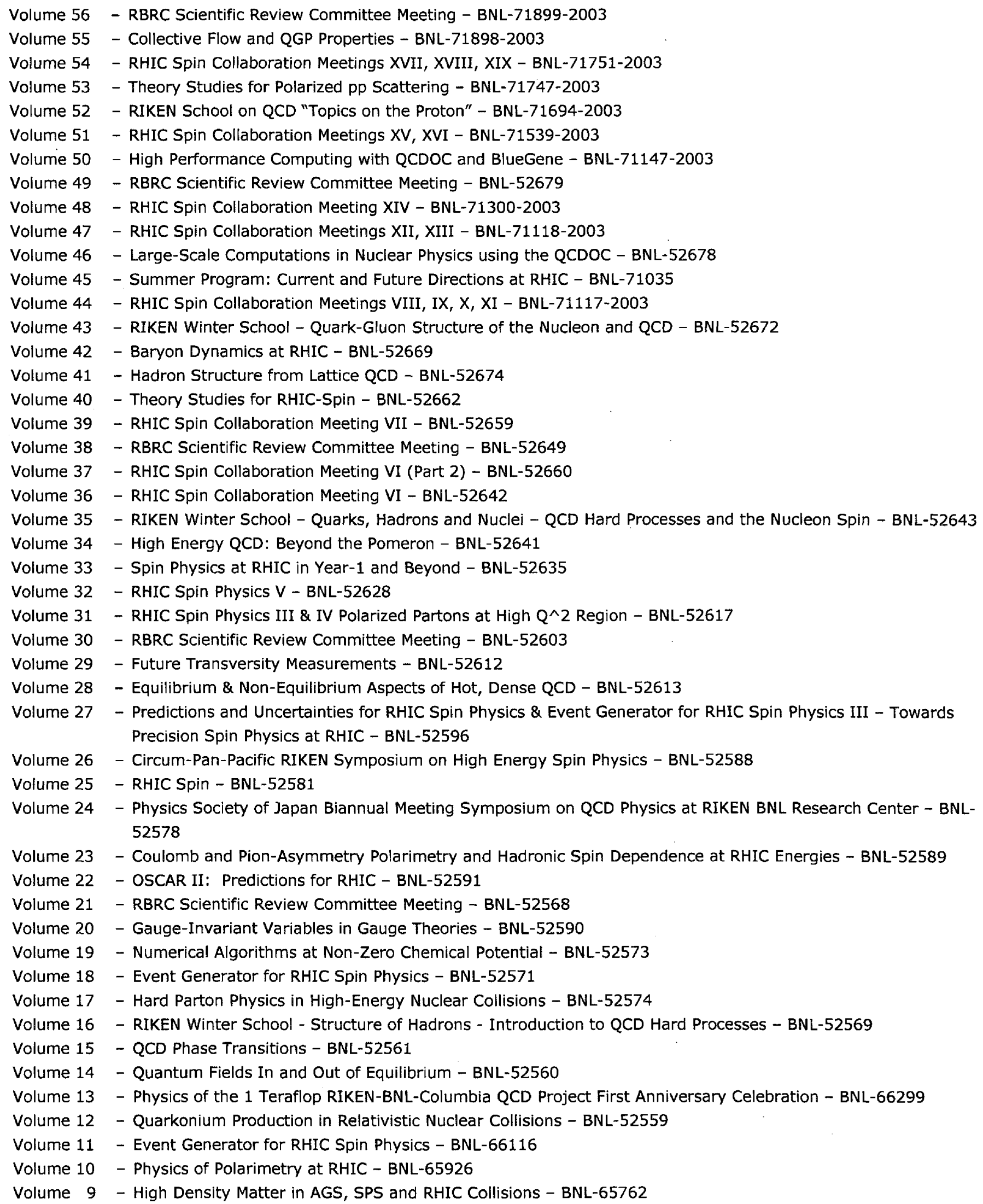




\section{Additional RIKEN BNL Research Center Proceedings:}

Volume 8 - Fermion Frontiers in Vector Lattice Gauge Theories - BNL-65634

Volume 7- RHIC Spin Physics - BNL-65615

Volume 6 - Quarks and Gluons in the Nucleon - BNL-65234

Volume 5 - Color Superconductivity, Instantons and Parity (Non?)-Conservation at High Baryon Density - BNL-65105

Volume 4 - Inauguration Ceremony, September 22 and Non-Equilibrium Many Body Dynamics -BNL-64912

Volume 3 - Hadron Spin-Flip at RHIC Energies - BNL-64724

Volume 2 - Perturbative QCD as a Probe of Hadron Structure - BNL-64723

Volume 1 - Open Standards for Cascade Models for RHIC - BNL-64722 



\section{For information please contact:}

Ms. Pamela Esposito

RIKEN BNL Research Center

Building 510A

Brookhaven National Laboratory

Upton, NY 11973-5000 USA

Phone: (631) 344-3097

Fax: (631) 344-4067

E-Mail: pesposit@bnl.gov
Ms. Susan Foster

RIKEN BNL Research Center

Building 510A

Brookhaven National Laboratory

Upton, NY 11973-5000 USA

(631) 344-5864

(631) 344-2562

sfoster@bnl.gov

Homepage: http://www.bnl.gov/riken 


\section{RIKEN BNL RESEARCH CENTER}

\section{The Physics of $\mathrm{W}$ and $\mathrm{Z}$ Bosons}

June 24-25, 2010

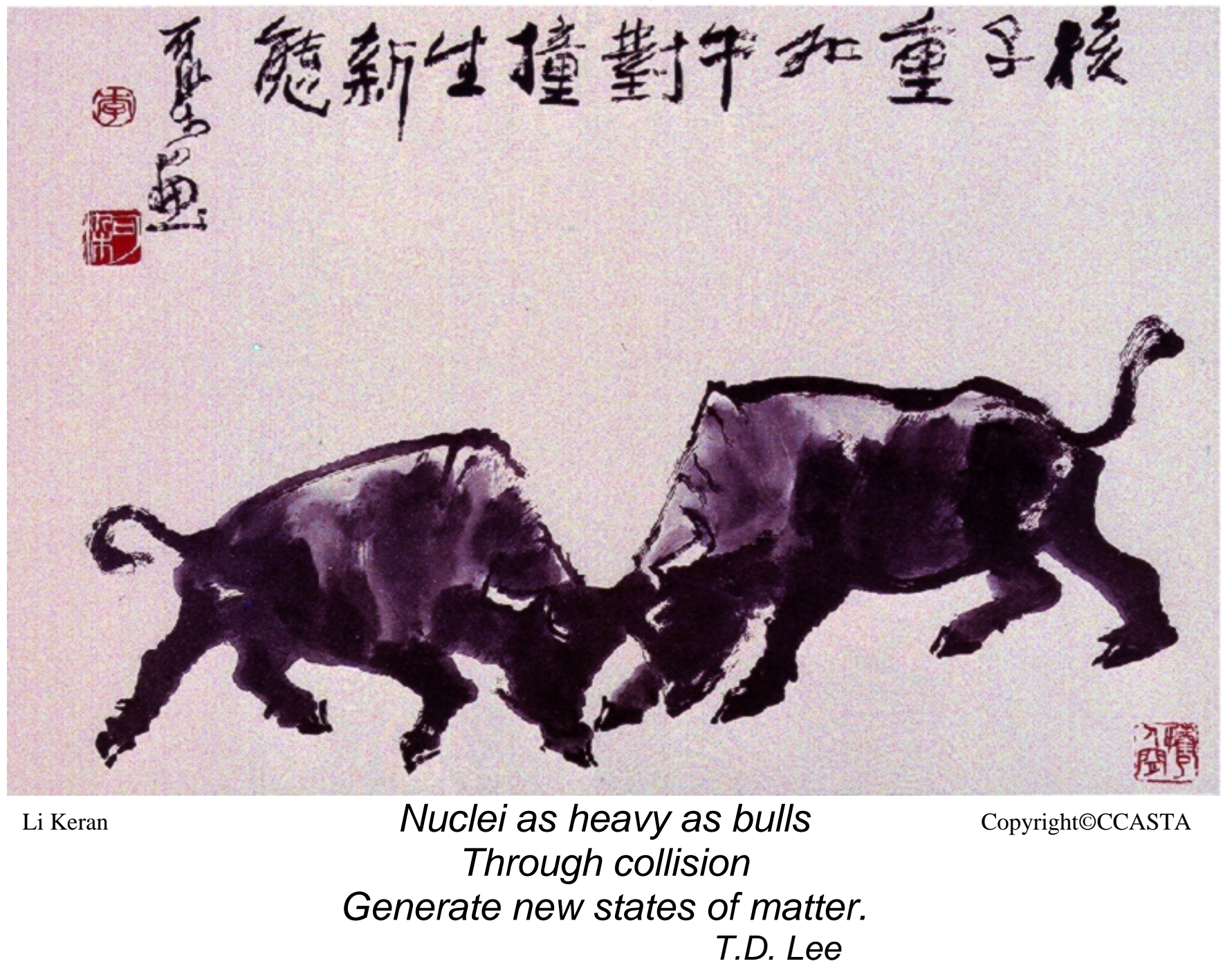

Speakers:

Jan Balewski

Yoshi Fukao

Ashutosh Kotwal

Francis Petriello

Jan Stark

C.-P. Yuan
Ulrich Baur

Zhongbo Kang

Carsten Magass

Joseph Seele

Alessandro Vicini

Junjie Zhu
Kevin Black

David Kawall

Pavel Nadolsky

David South

Jan Winter 